\title{
ON THE NON-LINEAR COHOMOLOGY OF LIE EQUATIONS. V
}

\author{
HUBERT GOLDSCHMIDT
}

TABLE OF CONTENTS

INTRODUCTION

\section{CHAPTER V. THE COHOMOLOGY OF NON-ABELIAN MINIMAL CLOSED IDEALS OF REAL TYPE}

21. Lie algebras with parameters

22. A class of Lie equations determined by forms

23. Some simple Lie algebras with parameters

24. Finite-dimensional Lie algebras with parameters

25. The contact algebra with parameters

26. The cohomology of non-abelian minimal closed ideals of real type

\section{INTRODUCTION}

This paper is a continuation of the joint papers with Donald Spencer, parts I and II of the same title which appeared in Acta Math. 136 (1976) 103-239 and parts III and IV of the same title which appeared in this journal 13 (1978) 409-526. We continue our study of the integrability problem for pseudogroups or for Lie equations and the program embarked upon in parts I and II and outlined in [17] for proving the solvability of the integrability problem for all Lie pseudogroups acting on $\mathbf{R}^{n}$ which contain the translations. Our proof follows to a large extent Guillemin's program for solving the integrability problem for flat pseudogroups and relies on Galois theory type methods similar to those introduced by Sophus Lie in his work on partial differential equations. In this work, these methods provide us with solutions of the non-linear partial differential equations associated to this integrability problem.

Communicated by D. C. Spencer, March 29, 1980. This work was supported in part by National Science Foundation Grants MCS 78-02459 and MCS 79-04683. 
In parts I and II of this paper, we started a program announced in [14] of studying the integrability problem in terms of transitive Lie algebras. If $R_{k}$ is a Lie equation on a manifold $X$ and $x \in X$, we defined a non-linear cohomology $\tilde{H}^{1}\left(R_{k}\right)_{x}$, which is a set with a distinguished element 0 . The integrability problem for $R_{k}$ (for a precise formulation of the problem, see [33]) is solvable if and only if $\tilde{H}^{1}\left(R_{k}\right)_{x}=0$ for all $x \in X$. If $R_{k}$ is formally transitive, this non-linear cohomology depends only on the transitive Lie algebra $R_{\infty, x}$ of formal solutions of $R_{k}$ at $x$. To a real transitive Lie algebra $L$ and to a closed ideal $I$ of $L$, we associated non-linear cohomologies $\tilde{H}^{1}(L)$ and $\tilde{H}^{1}(L, I)$ in such a way that $\tilde{H}^{1}(L, L)=\tilde{H}^{1}(L)$, and $\tilde{H}^{1}\left(R_{\infty, x}\right)=\tilde{H}^{1}\left(R_{k}\right)_{x}$ whenever $R_{k}$ is analytic and formally transitive. The integrability problem for formally transitive Lie equations is reduced to the study of the non-linear cohomology of transitive Lie algebras and their closed ideals. If $I$ is a closed ideal of the transitive Lie algebra $L$, the quotient $L / I$ is again a transitive Lie algebra and we proved that, if $\tilde{H}^{1}(L, I)=0$ and $\tilde{H}^{1}(L / I)=0$, then $\tilde{H}^{1}(L)=0$. By repeated applications of this result, if we consider a Jordan-Hölder sequence of Guillemin [20] for $L$, that is, a descending chain

$$
L=I_{0} \supset I_{1} \supset \cdots \supset I_{k}=0
$$

of closed ideals of $L$ such that, for $0 \leqslant j \leqslant k-1$, either $I_{j} / I_{j+1}$ is abelian or there are no closed ideals of $L$ properly contained between $I_{j}$ and $I_{j+1}$, it follows that to prove that $\tilde{H}^{1}(L)=0$ it suffices to show that $\tilde{H}^{1}\left(L / I_{j+1}, I_{j} / I_{j+1}\right)=0$ for the closed ideal $I_{j} / I_{j+1}$ of the transitive Lie algebra $L / I_{j+1}$, with $0 \leqslant j \leqslant k-1$; clearly the ideal $I_{j} / I_{j+1}$ is either a closed abelian ideal or a non-abelian minimal closed ideal of $L / I_{j+1}$. We are therefore lead to study the cohomology $\tilde{H}^{1}(L, I)$ of a closed abelian ideal or a non-abelian minimal closed ideal $I$ of $L$. In $\S 11$ and $\S 18$, we proved that, if $I$ is an abelian ideal of a transitive Lie algebra $L$, the non-linear cohomology $\tilde{H}^{1}(L, I)$ is isomorphic to the linear Spencer cohomology $H^{1}(L, I)$ introduced in [16] and that the vanishing of $\tilde{H}^{1}(L, I)$ is equivalent to the local solvability of a linear differential operator associated to $I$.

In this paper we turn our attention to the study of the non-linear cohomology $\tilde{H}^{1}(L, I)$ of a non-abelian minimal closed ideal $I$ of a real transitive Lie algebra $L$. According to [20], $I$ possesses a unique maximal closed ideal $J$ of $I$ and $R=I / J$ is the non-abelian and simple transitive Lie algebra canonically associated to $I$. The commutator ring $K_{R}$ of $R$ is the algebra of all $\mathbf{R}$-linear endomorphisms of $R$ which commute with all inner derivations of $R$. By a result of [20], $K_{R}$ is a field, and we say that $I$ is of real or complex type according to whether $K_{R}$ is $\mathbf{R}$ or $\mathbf{C}$. In parts I and II, we conjectured that $\tilde{H}^{1}(L, I)=0$; the examples of Conn [5] of non-abelian minimal closed ideals 
of complex type for which this cohomology does not vanish show that this Conjecture I of $\S 13$ is false, as is Conjecture II. The main result of this paper (Theorem 26.2) is the proof of Conjecture I for a non-abelian minimal closed ideal of real type, namely:

Theorem I. Let $L$ be a real transitive Lie algebra and I a non-abelian minimal closed ideal of $L$ of real type. Then $H^{j}(L, I)=0$ for $j>0$ and $\tilde{H}^{1}(L, I)=0$.

In a sequel to this paper, we shall study the cohomology of non-abelian minimal closed ideals of complex type and present counterexamples to the integrability problem arising from these ideals generalizing those of Conn [5]. In [6], Conn associates to a non-abelian minimal closed ideal $I$ of complex type of a real transitive Lie algebra $L$ an algebraic invariant, the Levi form, which is a vector-valued Hermitian form. In particular, we shall present a proof of

Theorem II. Let $L$ be a real transitive Lie algebra and I a non-abelian minimal closed ideal of $L$ of complex type. If the Levi form of I vanishes, then $H^{j}(L, I)=0$ for $j>0$ and $\tilde{H}^{1}(L, I)=0$.

We now briefly outline how the solvability of the integrability problem for a Lie pseudogroup acting on $\mathbf{R}^{n}$ which contains the translations can be deduced from Theorems I and II and the results of parts I and II. Let $L$ be a real transitive Lie algebra possessing a fundamental subalgebra $L^{0}$ and an abelian subalgebra $A$ such that

$$
L=L^{0} \oplus A .
$$

In fact, the transitive Lie algebra corresponding to such a pseudogroup has this property. Consider the Jordan-Hölder sequence for $L$ introduced above. In $\$ 11$ and $\S 13$, we proved that the linear differential operator associated to any abelian quotient $I_{j} / I_{j+1}$ is an operator with constant coefficients and we deduced from the Ehrenpreis-Malgrange theorem that $\tilde{H}^{1}\left(L / I_{j+1}, I_{j} / I_{j+1}\right)=$ 0 . If $I_{j} / I_{j+1}$ is a non-abelian minimal closed ideal of complex type of $L / I_{j+1}$, then the Levi form of $I_{j} / I_{j+1}$ vanishes. Therefore Theorems I and II imply that $\tilde{H}^{1}\left(L / I_{j+1}, I_{j} / I_{j+1}\right)=0$ whenever $I_{j} / I_{j+1}$ is a non-abelian minimal closed ideal of $L / I_{j+1}$. From these results, we deduce that $\tilde{H}^{1}(L)=0$ and that the integrability problem for pseudogroups acting on $\mathbf{R}^{n}$ containing the translations is solved. Furthermore these methods also give us a proof of Conjecture III of $\S 13$.

We now present an outline of the reduction of Theorem I to Theorem 26.1 which is based on $\S 13$ and is given here in $\$ 26$. Let $L$ be a real transitive Lie algebra and $I$ a non-abelian minimal closed ideal of $L$ of real type. According to the classification of the real simple infinite-dimensional transitive Lie algebras (see [21], [22], [34] and [36]), the Lie algebra $\operatorname{Der}(R)$ of derivations of 
the simple transitive Lie algebra $R$ associated to $I$ possesses a natural structure of transitive Lie algebra in which $R$ is a closed ideal of codimension at most one. Moreover $\operatorname{Der}(R)$ has a fundamental subalgebra $\operatorname{Der}^{0}(R)$ such that $R^{0}=R \cap \operatorname{Der}^{0}(R)$ is a fundamental subalgebra of $R$ and

$$
\operatorname{Der}(R)=R+\operatorname{Der}^{0}(R)
$$

(see [6]). The normalizer $N$ of the unique maximal closed ideal of $J$ of $I$ in $L$ is an open subalgebra of $L$. Let $F$ be the local ring of formal power series on the finite-dimensional vector space $(L / N)^{*}$ endowed with the Krull topology. The Lie algebra $\operatorname{Der}(F)$ of derivations of $F$ has a natural structure of transitive Lie algebra; if $F^{0}$ denotes the unique maximal ideal of $F$, the subalgebra $\operatorname{Der}^{0}(F)$ of $\operatorname{Der}(F)$ consisting of all elements $\xi$ of $\operatorname{Der}(F)$ satisfying $\xi\left(F^{0}\right) \subset F^{0}$ is a fundamental subalgebra of $\operatorname{Der}(F)$. Since $\operatorname{Der}(R)$ is a transitive Lie algebra and $F$ is a linearly compact topological algebra, there is a structure of linearly compact Lie algebra on the Hausdorff completion $\operatorname{Der}(R) \otimes_{\mathbf{R}} F$ of $\operatorname{Der}(R) \otimes_{\mathbf{R}} F$. The action of $\operatorname{Der}(F)$ on $F$ determines a structure of linearly compact $\operatorname{Der}(F)$-module on $\operatorname{Der}(R) \hat{\otimes}_{\mathbf{R}} F$, the semi-direct product

$$
\operatorname{Der}\left(R \hat{\otimes}_{\mathbf{R}} F\right)=\left(\operatorname{Der}(R) \hat{\otimes}_{\mathbf{R}} F\right) \oplus \operatorname{Der}(F)
$$

has a natural structure of real transitive Lie algebra,

$$
\operatorname{Der}^{0}\left(R \hat{\otimes}_{\mathbf{R}} F\right)=\left(\operatorname{Der}^{0}(R) \hat{\otimes}_{\mathbf{R}} F+\operatorname{Der}(R) \hat{\otimes}_{\mathbf{R}} F^{0}\right) \oplus \operatorname{Der}^{0}(F)
$$

is a fundamental subalgebra, and $R \hat{\otimes}_{\mathbf{R}} F$ is a non-abelian minimal closed ideal of this Lie algebra; we call the ideal $R \hat{\otimes}_{\mathbf{R}} F$ the Lie algebra $R$ with real parameters (see [6]). According to the topological version of Conn [6] of the structure theorem of Guillemin [20], there is a continuous morphism of transitive Lie algebras

$$
\Phi: L \rightarrow \operatorname{Der}\left(R \hat{\otimes}_{\mathbf{R}} F\right)
$$

whose restriction to $I$ is an isomorphism

$$
\Phi: I \rightarrow R \hat{\otimes}_{\mathbf{R}} F
$$

such that

$$
\operatorname{Der}\left(R \hat{\otimes}_{\mathbf{R}} F\right)=\Phi(L)+\operatorname{Der}^{0}\left(R \hat{\otimes}_{\mathbf{R}} F\right) .
$$

By results of [16] and $\S 10, \Phi$ induces isomorphisms of cohomology

$$
\begin{gathered}
H^{*}(L, I) \rightarrow H^{*}\left(\operatorname{Der}\left(R \hat{\otimes}_{\mathbf{R}} F\right), R \hat{\otimes}_{\mathbf{R}} F\right), \\
\tilde{H}^{1}(L, I) \rightarrow \tilde{H}^{1}\left(\operatorname{Der}\left(R \hat{\otimes}_{\mathbf{R}} F\right), R \hat{\otimes}_{\mathbf{R}} F\right) .
\end{gathered}
$$

Theorem I follows from Theorem 26.1 which asserts that

$$
H^{j}\left(\operatorname{Der}\left(R \hat{\otimes}_{\mathbf{R}} F\right), R \hat{\otimes}_{\mathbf{R}} F\right)=0, \quad \tilde{H}^{1}\left(\operatorname{Der}\left(R \hat{\otimes}_{\mathbf{R}} F\right), R \hat{\otimes}_{\mathbf{R}} F\right)=0,
$$


for $j>0$, where $R$ is a simple transitive Lie algebra, with $K_{R}=\mathbf{R}$, and $F$ is the ring of formal power series on a finite-dimensional vector space $U$.

In $\$ \S 22-25$ to prove Theorem 26.1 , for each simple transitive Lie algebra $R$, with $K_{R}=\mathbf{R}$, and each finite-dimensional vector space $U$, we construct explicitly a formally integrable and formally transitive analytic Lie equation $R_{k}^{\#}$ of order $k$, with $k=1$ or 2 depending on whether $\operatorname{Der}(R)$ equals $R$ or not, on an analytic manifold $X$, a formally integrable analytic first-order Lie equation $R_{1}$ such that $R_{\infty, a}$ is a closed ideal of $R_{\infty, a}^{\#}$ for all $a \in X$, a formally integrable and integrable finite form $P_{1}$ of $R_{1}$ and an isomorphism of transitive Lie algebras $\psi: R_{\infty, x}^{\sharp} \rightarrow \operatorname{Der}\left(R \hat{\otimes}_{\mathbf{R}} F\right)$ such that $\psi\left(R_{\infty, x}\right)=R \hat{\otimes}_{\mathbf{R}} F$, where $x$ is a point of $X$. Then we show that the linear Spencer cohomology $H^{j}\left(R_{1}\right)$ and the non-linear cohomology $\bar{H}^{1}\left(P_{1}\right)_{a}$, defined in terms of the $\overline{\mathfrak{D}}$-complex (see $\S 7$ ), vanish for all $j>0$ and $a \in X$; it follows that the assertions of Theorem 26.1 hold for $R$ and $U$. If $J_{k}(T)$ is the bundle of $k$-jets of the tangent bundle $T$ of $X$, the Lie equation $R_{1}$ is a sub-bundle of $J_{1}(T)$ and $P_{1}$ is a sub-bundle of the bundle $Q_{1}$ of 1-jets of local diffeomorphisms of $X$. If $u$ is a section of $T^{*} \otimes J_{1}(T)$, we denote by $\pi_{0} u$ the section of $T^{*} \otimes J_{0}(T)$ determined by $u$. Let $\mathfrak{D}_{1}$ be the compatibility condition for the operator $\mathfrak{D}$ of the non-linear Spencer complex; the set $Z^{1}\left(R_{1}\right)_{a}$ of $\mathfrak{D}_{1}$-cocycles at $a \in X$ consists of the germs at $a$ of sections $u$ of $T^{*} \otimes R_{1}$ which satisfy $\mathfrak{D}_{1} u=0$ and $\pi_{0} u(a)$ close to 0 . The vanishing of the non-linear cohomology $\bar{H}^{1}\left(P_{1}\right)_{a}$ is proved using the following necessary and sufficient condition given by Proposition 22.7: for all $u \in$ $Z^{1}\left(R_{1}\right)_{a}$ we can solve the equation $\mathfrak{D} \phi=\pi_{0} u$ for some germ $\phi$ of an invertible section of $P_{1}$.

According to the classification results referred to above, the simple transitive Lie algebra $R$ is either finite-dimensional or isomorphic to one of the following:

(i) the Lie algebra of all formal vector fields on $\mathbf{R}^{n}$;

(ii) the Lie algebra of all formal vector fields with zero-divergence on $\mathbf{R}^{n}$, with $n \geqslant 2$;

(iii) the Lie algebra of all formal symplectic vector fields on $\mathbf{R}^{2 n}$, with $n \geqslant 1$;

(iv) the Lie algebra of all contact vector fields on $\mathbf{R}^{2 n+1}$, with $n \geqslant 1$.

If $R$ is equal to one of the transitive Lie algebras (ii) or (iii), these constructions are made in $\$ 23$ and the corresponding vanishing of the linear and non-linear cohomology is proved in Theorem 23.1, while the analogues for the Lie algebra (iv) are accomplished in $\$ 25$ and Theorem 25.1. The case of a finite-dimensional Lie algebra $R$ is considered in $\$ 24$ and the vanishing of cohomology for the Lie algebra (i) is given by Proposition 22.9.

We give a unified treatment of the Lie algebras (i)-(iii) and of finite-dimensional Lie algebras in terms of the class of Lie equations whose solutions 
preserve a differential form. Let $X, Y$ be analytic manifolds and $\rho: X \rightarrow Y$ an analytic surjective submersion. We assume that the dimension of $Y$ is equal to that of the vector space $U$. In $\S 22$, under certain regularity assumptions, we associate to a differential form $\omega$ along the fibers of $\rho$ a first-order Lie equation $J_{1}(V ; \omega)$ on the bundle $V$ of vectors tangent to the fibers of $\rho$ and a finite form $Q_{1}(V ; \omega)$ of $J_{1}(V ; \omega)$. The solutions of $J_{1}(V ; \omega)$ are the vertical (with respect to $\rho$ ) vector fields $\xi$ on $X$ which preserve $\omega$, that is, for which the Lie derivative $\mathcal{L}(\xi) \omega$ of $\omega$ along $\xi$ vanishes. If $J_{k}(V)$ denotes the bundle of $k$-jets of $V$, a section $u_{0}$ of $T^{*} \otimes J_{0}(V)$ operates on the space of differential forms along the fibers of $\rho$ and associates to $\omega$ the differential form $\omega^{u_{0}}$. If $u$ is a section of $T^{*} \otimes J_{1}(V)$, the fundamental formula (22.20) relates the actions of the section $u_{0}=\pi_{0} u$ of $T^{*} \otimes J_{0}(V)$ determined by $u$ and of the exterior differential operator $d_{X / Y}$ on differential forms along the fibers of $\rho$ to $\mathfrak{D}_{1} u$. We denote by $\tilde{\mathscr{Q}}_{1}$ the sheaf of invertible sections of $Q_{1}$. If $J_{1}(V ; \omega)$ is formally integrable and $Q_{1}(V ; \omega)$ is a formally integrable and integrable differential equation, in Proposition 22.8 we obtain as a consequence of Proposition 22.7 a criterion for the vanishing of the non-linear cohomology of $Q_{1}(V ; \omega)$ or of $J_{1}(V ; \omega)$ at $a \in X$ : if $u \in Z^{1}\left(J_{1}(V ; \omega)\right)_{a}$, it suffices to find a germ $f$ at $a$ of a local diffeomorphism of $X$ over $Y$ such that

$$
f^{*} \omega=\omega^{u_{0}},
$$

where $u_{0}=\pi_{0} u$; in fact the relation (1) guarantees that the unique element $\phi \in \tilde{\mathscr{Q}}_{1}$ satisfying $\pi_{0} \phi=f$ and $\mathfrak{D} \phi=u_{0}$ is a germ of a section of $Q_{1}(V ; \omega)$.

In $\S 23$, we suppose that $\omega$ is an analytic volume or symplectic form along the fibers of $\rho$; we prove that $J_{1}(V ; \omega)$ is a formally integrable and involutive Lie equation and that $Q_{1}(V ; \omega)$ is a formally integrable and integrable finite form of $J_{1}(V ; \omega)$ (Proposition 23.3). If $\omega$ is a symplectic (resp. volume) form and $u \in Z^{1}\left(J_{1}(V ; \omega)\right)_{a}$, with $a \in X$ and $u_{0}=\pi_{0} u$, then $\omega^{u_{0}}$ is closed according to the fundamental formula (22.20) and is the germ of a symplectic (resp. volume) form along the fibers of $\rho$; moreover every such germ can be obtained in this way. Darboux's theorem with parameters (resp. an elementary result about ordinary differential equations) gives us the existence of a germ $f$ at $a$ of a local diffeomorphism of $X$ over $Y$ satisfying $f^{*} \omega=\omega^{u_{0}}$, and hence the vanishing of the non-linear cohomology of $Q_{1}(V ; \omega)$ at $a$. A $Q_{1}(V ; \omega)$-structure on $X$ is equivalent to a non-singular differential 2-form (resp. $q$-form, where $q=$ rank $V$ ) along the fibers of $\rho$ and is formally integrable in the sense of Malgrange [33] if and only if this differential form is closed, and hence is a symplectic form (resp. is always formally integrable). If $\omega$ is a symplectic form, this last remark can be used to derive directly the fact that $\omega^{u_{0}}$ is a germ of a symplectic form for $u \in Z^{1}\left(J_{1}(V ; \omega)\right)_{a}$ and $u_{0}=\pi_{0} u$ without requiring our 
formula (22.20). Finally, we construct a second-order formally integrable and formally trainsitive analytic Lie equation $J_{2}^{\prime}(T ; \omega)$ on $X$ whose solutions over connected open subsets of $X$ are the $\rho$-projectable vector fields $\xi$ satisfying the relation $\mathcal{L}(\xi) \omega=\rho^{*} g \cdot \omega$, for some real-valued function $g$ on $Y$. If rank $V \geqslant 2$, for $x \in X$ we verify that $J_{\infty}(V ; \omega)_{x}$ is a closed ideal of the transitive Lie algebra $J_{\infty}^{\prime}(T ; \omega)_{x}$ and that, if $R$ is the Lie algebra (iii) with $2 n=\operatorname{rank} V$ (resp. the Lie algebra (ii) with $n=\operatorname{rank} V$ ), there is an isomorphism of transitive Lie algebras

$$
\psi: J_{\infty}^{\prime}(T ; \omega)_{x} \rightarrow \operatorname{Der}\left(R \hat{\otimes}_{\mathbf{R}} F\right)
$$

such that $\psi\left(J_{\infty}(V ; \omega)_{x}\right)=R \hat{\otimes}_{\mathbf{R}} F$; it follows that the non-linear cohomology of the closed ideal $R \hat{\otimes}_{\mathrm{R}} F$ of $\operatorname{Der}\left(R \hat{\otimes}_{\mathrm{R}} F\right)$ vanishes.

If $R$ is a finite-dimensional Lie algebra, in $\$ 24$ we consider a Lie group $G_{0}$ whose Lie algebra $g_{0}$ is isomorphic to $R$. If $X=Y \times G_{0}$ and $\rho: X \rightarrow Y$ is the projection onto the second factor, the left-invariant and right-invariant Maurer-Cartan forms of $G_{0}$ induce non-singular $g_{0}$-valued 1-forms $\omega$ and $\sigma$ respectively along the fibers of $\rho$. From the identity (24.4) relating the Lie derivative $\mathcal{L}(\xi) \omega$ of $\omega$ along a vertical vector field $\xi$ on $X$ to the exterior derivative $d_{X / Y}\langle\sigma, \xi\rangle$ of the $g_{0}$-valued function $\langle\sigma, \xi\rangle$, we deduce that $J_{1}(V ; \omega)$ is a formally integrable Lie equation whose solutions over open subsets of $X$ whose fibers over $Y$ are connected are the vertical vector fields whose restrictions to each fiber are right-invariant vector fields on $G_{0}$. From the formula (24.7) relating the first-order (non-linear) differential operators $\mathfrak{D}_{X / Y}$ of $\S 4$ and $\mathfrak{D}_{\omega}$, which corresponds to the equation $Q_{1}(V ; \omega)$, we infer that the solutions of $Q_{1}(V ; \omega)$ over open subsets of $X$ whose fibers over $Y$ are connected are the local diffeomorphisms of $X$ whose restrictions to each fiber are left-translations of $G_{0}$ and, using properties of the operator $\mathfrak{D}_{X / Y}$, that $Q_{1}(V ; \omega)$ is a formally integrable and integrable finite form of $J_{1}(V ; \omega)$ (Proposition 24.3). If $\mathfrak{D}_{1, X / Y}$ is the compatibility condition for the operator $\mathfrak{D}_{X / Y}$, the Maurer-Cartan equation satisfied by $\omega$ is $\mathfrak{D}_{1, X / Y} \omega=0$. Using this fact and the fundamental formula (22.20), we obtain the commutative diagram (24.17) connecting the non-linear Spencer $\mathfrak{D}$-complex of $J_{1}(V ; \omega)$ and the sequence of $\S 4$ involving the operators $\mathfrak{D}_{X / Y}$ and $\mathfrak{D}_{1, X / Y}$. If $u \in Z^{1}\left(J_{1}(V ; \omega)\right)_{a}$, with $a \in X$ and $u_{0}=\pi_{0} u$, then $\omega^{u_{0}}$ is a germ of a non-singular $g_{0}$-valued 1-form along the fibers of $\rho$ satisfying the equation $\mathfrak{D}_{1, X / Y} \omega^{u_{0}}=0$; moreover every such germ can be obtained in this way. Proposition 4.1, which is a consequence of Frobenius' theorem, says that this sequence of $\S 4$ is exact and hence gives us a germ at $a$ of a local diffeomorphism $f$ of $X$ over $Y$ satisfying $\mathfrak{D}_{X / Y} f=\omega^{u_{0}}$, which is precisely equation (1), and the vanishing of the non-linear cohomology of $Q_{1}(V ; \omega)$ at $a$. The exactness of this sequence is the 
assertion of the second fundamental theorem with parameters for the Lie group $G_{0}$. A $Q_{1}(V ; \omega)$-structure on $X$ is equivalent to a non-singular $\mathrm{g}_{0}$-valued 1 -form $v$ along the fibers of $\rho$ and is formally integrable if and only if $\mathfrak{D}_{1, X / Y} v=0$. This last remark can be used to show directly that $\mathfrak{D}_{1, X / Y} \omega^{u_{0}}=0$ for $u \in Z^{1}\left(J_{1}(V ; \omega)\right)_{a}$ and $u_{0}=\pi_{0} u$ without requiring our formula (22.20). The analytic first-order Lie equation $J_{1}(T ; \omega)$ on $X$, whose solutions are the $\rho$-projectable vector fields $\xi$ satisfying $\mathscr{L}(\xi) \omega=0$, is formally integrable and formally transitive. For $x \in X$, we verify that $J_{\infty}(V ; \omega)_{x}$ is a closed ideal of the transitive Lie algebra $J_{\infty}(T ; \omega)_{x}$ and that, if $R$ is simple, there is an isomorphism of transitive Lie algebras

$$
\psi: J_{\infty}(T ; \omega)_{x} \rightarrow \operatorname{Der}\left(R \hat{\otimes}_{\mathbf{R}} F\right)
$$

such that $\psi\left(J_{\infty}(V ; \omega)_{x}\right)=R \hat{\otimes}_{\mathbf{R}} F$. Thus the non-linear cohomology considered in Theorem 26.1 vanishes whenever $R$ is finite-dimensional. We remark that, if $U$ is zero-dimensional and $Y$ is a point, the Lie equation $J_{1}(V ; \omega)$ and its finite form $Q_{1}(V ; \omega)$ can be described in a much simpler way without the use of $\omega$ (see $\$ 20$ ) and the vanishing of the cohomology in this case is given by Proposition 17.2.

In $\$ 25$, we study the case of the Lie algebra (iv). If $\omega$ is an analytic contact form along the fibers of $\rho$, the Lie equation $J_{1}(V ; W)$, corresponding to the equation $(\mathscr{L}(\xi) \omega) \wedge \omega=0$ for vertical vector fields $\xi$, whose solutions are the contact vector fields along the fibers of $\rho$, is not formally integrable. We introduce a first-order Lie equation $R_{1}(V ; \omega)$ (resp. $\left.R_{1}(\omega)\right)$ which corresponds to the equations

$$
\mathcal{L}(\xi) \omega=f \omega, \quad \mathcal{L}(\xi) d_{X / Y} \omega=f d_{X / Y} \omega+\alpha \wedge \omega
$$

for the vertical (resp. $\rho$-projectable) vector field $\xi$, where $f$ is a real-valued function on $X$ and $\alpha$ is a 1-form along the fibers of $\rho$ depending on $\xi$. The solutions of $R_{1}(V ; \omega)$ are precisely the contact vector fields along the fibers of $\rho$. In Proposition 25.2, using the explicit form of the contact vector fields, we show that this equation $R_{1}(V ; \omega)$ is formally integrable and integrable and that it is involutive and can be obtained from $J_{1}(V ; W)$ by the methods of [10]; in fact, it is equal to the projection in $J_{1}(V)$ of the first prolongation $J_{2}(V ; W)$ of the equation $J_{1}(V ; W)$. This enables us to apply the results of [10] to deduce that $H^{j}\left(R_{1}(V ; \omega)\right)=0$, for $j>0$. To verify that a certain differential equation $P_{1}(V ; \omega) \subset Q_{1}$ is a formally integrable and integrable finite form of $R_{1}(V ; \omega)$, we are lead to examine properties of a finite form $Q_{1}(V ; W)$ of $J_{1}(V ; W)$ and then to construct explicitly elements of the first prolongation $P_{2}(V ; \omega)$ of $P_{1}(V ; \omega)$ in order to apply results of [9]. If $u \in Z^{1}\left(R_{1}(V ; \omega)\right)_{a}$, with $a \in X$ and 
$u_{0}=\pi_{0} u$, then by means of the formula (22.20) we see that $\omega^{u_{0}}$ is a germ of a 1 -form along the fibers of $\rho$ satisfying the condition

$$
\omega^{u_{0}} \wedge\left(d_{X / Y} \omega^{u_{0}}-\left(d_{X / Y} \omega\right)^{u_{0}}\right)=0 ;
$$

in fact the relation (2) implies that $\omega^{u_{0}}$ is a germ of a contact form and moreover every germ of a contact form along the fibers of $\rho$ can be obtained in this way (Propositions 25.5 and 25.6). Darboux's theorem with parameters gives us the existence of a germ at $a$ of a local diffeomorphism $f$ of $X$ over $Y$ satisfying (1); from equation (2) we infer that $f$ verifies the additional relation

$$
\omega^{u_{0}} \wedge\left(f^{*} d_{X / Y} \omega-\left(d_{X / Y} \omega\right)^{u_{0}}\right)=0 .
$$

The equations (1) and (3) guarantee that the unique element $\phi \in \tilde{\mathcal{Q}}_{1}$ satisfying $\pi_{0} \phi=f$ and $\mathscr{D} \phi=u_{0}$ is a germ of a section of $P_{1}(V ; \omega)$ (Proposition 25.4). Our criterion now insures that the non-linear cohomology of $P_{1}(V ; \omega)$ vanishes. The equation (2) expresses the condition that a germ $u_{0}$ at $a$ of a section of $T^{*} \otimes J_{0}(V)$, with $u_{0}(a)$ close to 0 , be of the form $u_{0}=\pi_{0} u$ for some $u \in$ $Z^{1}\left(R_{1}(V ; \omega)\right)_{a}$. Finally, the analytic Lie equation $R_{1}(\omega)$ is formally integrable and formally transitive; for $x \in X$, we verify that $R_{\infty}(V ; \omega)_{x}$ is a closed ideal of the transitive Lie algebra $R_{\infty}(\omega)_{x}$ and that, if $R$ is the Lie algebra (iv) with $2 n+1=\operatorname{rank} V$, there is an isomorphism of transitive Lie algebras

$$
\psi: R_{\infty}(\omega)_{x} \rightarrow \operatorname{Der}\left(R \hat{\otimes}_{\mathbf{R}} F\right)
$$

such that $\psi\left(R_{\infty}(V ; \omega)_{x}\right)=R \hat{\otimes}_{\mathbf{R}} F$. It follows that the assertions of Theorem 26.1 hold whenever $R$ is the Lie algebra (iv).

Finally, we ought to point out to the reader that all differential equations considered throughout this paper are assumed to be of order greater than or equal to one. We wish to thank D. C. Spencer for his constant encouragement and advice during the preparation of this paper.

\section{CHAPTER V. THE COHOMOLOGY OF NON-ABELIAN MINIMAL CLOSED IDEALS OF REAL TYPE}

\section{Lie Algebras with parameters}

Let $K$ be a field endowed with the discrete topology. We begin by recalling the following definitions.

Definition. A linearly compact Lie algebra over $K$ is simple if it contains no non-trivial ideals.

According to [20, Proposition 4.3] a linearly compact Lie algebra is simple if and only if it contains no non-trivial closed ideals; clearly such a simple Lie algebra is transitive. 
Definition. Let $L$ be a transitive Lie algebra over $K$ and $I$ a closed ideal of $L$. A Jordan-Hölder sequence for $(L, I)$ of length $k$ is a properly nested chain

$$
I=I_{0} \supset I_{1} \supset I_{2} \supset \cdots \supset I_{k}=0
$$

of closed ideals of $L$ such that, for all $0 \leqslant j \leqslant k-1$, either $I_{j} / I_{j+1}$ is abelian or there are no closed ideals of $L$ properly contained between $I_{j}$ and $I_{j+1}$.

If $I$ is equal to $L$, we call such a descending chain a Jordan-Hölder sequence for $L$. The existence of such sequences for $(L, I)$ was proved by Guillemin [20, Theorem 6.1] (see also Theorem 12.2).

Let $L, E$ be linearly compact Lie algebras over $K$ and suppose that $E$ is endowed with the structure of a linearly compact $L$-module such that the elements of $L$ act on $E$ as derivations of the Lie algebra $E$, that is,

$$
\xi \cdot\left[\eta_{1}, \eta_{2}\right]=\left[\xi \cdot \eta_{1}, \eta_{2}\right]+\left[\eta_{1}, \xi \cdot \eta_{2}\right],
$$

for $\xi \in L, \eta_{1}, \eta_{2} \in E$. We define a structure of linearly compact Lie algebra $M$ on the linearly compact topological vector space $E \times L$ by setting

$$
\left[\left(\eta_{1}, \xi_{1}\right),\left(\eta_{2}, \xi_{2}\right)\right]=\left(\xi_{1} \cdot \eta_{2}-\xi_{2} \cdot \eta_{1}+\left[\eta_{1}, \eta_{2}\right],\left[\xi_{1}, \xi_{2}\right]\right)
$$

The Jacobi identity for $M$ follows from (21.1). We identify $E$ (resp. $L$ ) with its image in $M$ under the Lie algebra homomorphism $i: E \rightarrow M$ sending $\eta$ into $(\eta, 0)$ (resp. $j: L \rightarrow M$ sending $\xi$ into $(0, \xi)$ ). If $\phi: M \rightarrow L$ denotes the projection sending $(\eta, \xi) \in E \times L$ into $\xi$, the sequence

$$
0 \rightarrow E \stackrel{i}{\rightarrow} M \stackrel{\phi}{\rightarrow} L \rightarrow 0
$$

is an exact sequence of linearly compact Lie algebras which is split by $j$; moreover

$$
\xi \cdot \eta=[j(\xi), i(\eta)]
$$

for $\xi \in L, \eta \in E$. Thus $M$ is an inessential extension of $L$ by the closed ideal $E$ which we call the semi-direct product of $L$ and $E$ and often denote by $E \oplus L$. A closed Lie subalgebra $F \subset E$ which is also an $L$-submodule of $E$ determines a closed ideal $F$ of $M$.

Let $E, F$ be linearly compact topological vector spaces over $K$. If $\left\{E^{\alpha}\right\}_{\alpha \in A}$, $\left\{F^{\beta}\right\}_{\beta \in B}$ are fundamental systems of neighborhoods of 0 in $E$ and $F$ respectively, we endow the tensor product $E \otimes_{K} F$ with a structure of topological vector space by letting the subspaces

$$
E^{\alpha} \otimes_{K} F+E \otimes_{K} F^{\beta}
$$

with $\alpha \in A, \beta \in B$, of $E \otimes_{K} F$ be a fundamental system of neighborhoods of 0 . The Hausdorff completion of $E \otimes_{K} F$ is a linearly compact topological vector 
space which we denote by $E \hat{\otimes}_{K} F$; we have a canonical injective mapping

$$
E \otimes_{K} F \rightarrow E \hat{\otimes}_{K} F
$$

which enables us to identify $E \otimes_{K} F$ with a dense subspace of $E \hat{\otimes}_{K} F$. The tensor product $E \hat{\otimes}_{K} F$ may be canonically identified with the projective limits

$$
\lim _{\leftarrow}\left(E / E^{\alpha}\right) \otimes_{K}\left(F / F^{\beta}\right)=\lim _{\leftarrow}\left(E / E^{\alpha}\right) \otimes_{K} F=\lim _{\leftarrow} E \otimes_{K}\left(F / F^{\beta}\right) .
$$

If one of the spaces $E$ or $F$ is finite-dimensional, then $E \hat{\otimes}_{K} F$ coincides with $E \otimes_{K} F$.

Let $F$ be a linearly compact (associative) algebra over $K$, that is, a topological algebra over $K$ whose underlying topological vector space is linearly compact, and let $R$ be a linearly compact Lie algebra over $K$. The tensor product $R \otimes_{K} F$ has a structure of Lie algebra determined by

$$
\left[\xi_{1} \otimes f_{1}, \xi_{2} \otimes f_{2}\right]=\left[\xi_{1}, \xi_{2}\right] \otimes f_{1} \cdot f_{2},
$$

for $\xi_{1}, \xi_{2} \in R, f_{1}, f_{2} \in F$. Then it is easily verified that there is a unique topological Lie algebra structure on $R \hat{\otimes}_{K} F$ which extends this Lie algebra structure on $R \otimes_{K} F$. Let $L$ be a linearly compact Lie algebra. Assume that $F$ is a linearly compact $L$-module such that the elements of $L$ act on $F$ as derivations of the associative Lie algebra $F$, that is,

$$
\xi \cdot\left(f_{1} \cdot f_{2}\right)=\left(\xi \cdot f_{1}\right) \cdot f_{2}+f_{1} \cdot\left(\xi \cdot f_{2}\right),
$$

for $\xi \in L, f_{1}, f_{2} \in F$. The action of $L$ on $R \otimes_{K} F$, determined by

$$
\xi \cdot(\eta \otimes f)=\eta \otimes \xi \cdot f,
$$

for $\xi \in L, \eta \in R, f \in F$, extends to give us a structure of linearly compact $L$-module on $R \hat{\otimes}_{K} F$ such that (21.1) holds for $\xi \in L, \eta_{1}, \eta_{2} \in R \hat{\otimes}_{K} F$. Therefore we may define the semi-direct product of $L$ and $R \hat{\otimes} F$ according to the construction given above.

Let 1 be the trivial line bundle over the manifold $Y$ which is associated to $\tilde{J}_{1}\left(T_{Y} ; Y\right)$. Thus $F_{Y}=J_{\infty}(1 ; Y)_{y}$ is a geometric $J_{\infty}\left(T_{Y} ; Y\right)_{y}$-module, for $y \in Y$, and a linearly compact algebra, and the elements of $J_{\infty}\left(T_{Y} ; Y\right)_{y}$ act as derivations of the algebra $F_{y}$. We denote by $F_{y}^{0}$ the kernel of $\pi_{0}: J_{\infty}(1 ; Y)_{y} \rightarrow$ $J_{0}(1 ; Y)_{y}$. Let $L$ be a real transitive Lie algebra, $I$ a closed ideal of $L$ and $L^{\prime}$ a closed subalgebra of $L$. According to the above construction, we obtain the semi-direct product

$$
M=\left(L \hat{\otimes}_{\mathbf{R}} F_{y}\right) \oplus J_{\infty}\left(T_{Y} ; Y\right)_{y}
$$

of the transitive Lie algebra $J_{\infty}\left(T_{Y} ; Y\right)_{y}$ and the $J_{\infty}\left(T_{Y} ; Y\right)_{y}$-module $L \hat{\otimes}_{\mathbf{R}} F_{y}$. By [6, Lemma 3.1], $M$ is a transitive Lie algebra and, if $L^{0}$ is a fundamental subalgebra of $L$, then

$$
M^{0}=\left(L^{0} \hat{\otimes} F_{y}+L \hat{\otimes} F_{y}^{0}\right) \oplus J_{\infty}^{0}\left(T_{Y} ; Y\right)_{y}
$$


is a fundamental subalgebra of $M$. Moreover the $J_{\infty}\left(T_{Y} ; Y\right)_{y}$-module $I \hat{\otimes}_{\mathbf{R}} F_{y}$ is a closed ideal of $M$ and the semi-direct product

$$
\left(L^{\prime} \hat{\otimes} F_{y}\right) \oplus J_{\infty}\left(T_{Y} ; Y\right)_{y}
$$

is a closed subalgebra of $M$. We say that the ideal $I \hat{\otimes}_{\mathbf{R}} F_{y}$ of $M$ is the Lie algebra $I$ with real parameters. If the closed ideal $I$ of $L$ is a non-abelian and simple Lie algebra, then it is easily verified that $I \hat{\otimes}_{\mathbf{R}} F_{y}$ is a non-abelian minimal closed ideal of $M$. If $L$ is a non-abelian and simple Lie algebra, then as $J_{\infty}\left(T_{Y} ; Y\right)_{y}$ is a simple transitive Lie algebra when the dimension of $Y$ is $\geqslant 1$, we see that

$$
M \supset L \hat{\otimes}_{\mathbf{R}} F_{y} \supset 0
$$

is a Jordan-Hölder sequence for $M$.

Assume that the manifold $X$ is the product $Y \times Z$ of $Y$ with a manifold $Z$ and that $\rho: X \rightarrow Y$ is the projection onto the first factor. Since $T_{x}=T_{Y, y} \oplus T_{Z, z}$, if $x=(y, z) \in Y \times Z$, we have an isomorphism $T_{Z, z} \rightarrow V_{x}$; thus a vector field $\xi$ on $Y$ determines a $\rho$-projectable vector field $\rho^{-1} \xi$ on $X$, while a vector field $\eta$ on $Z$ determines a vector field $\operatorname{pr}_{2}^{-1} \eta$ on $X$, and a $p$-form on $Z$ determines a section of $\wedge^{p} V^{*}$ over $X$. On the other hand, we obtain a morphism of Lie algebras

$$
\sigma_{x}: J_{\infty}\left(T_{Y} ; Y\right)_{y} \rightarrow J_{\infty}(T ; \rho)_{x},
$$

sending $j_{\infty}(\xi)(y)$ into $j_{\infty}\left(\rho^{-1} \xi\right)(x)$, where $x \in X$ satisfies $\rho(x)=y$, which is a splitting of the exact sequence of Lie algebras

$$
0 \rightarrow J_{\infty}(V)_{x} \rightarrow J_{\infty}(T ; \rho)_{x} \stackrel{\rho}{\rightarrow} J_{\infty}\left(T_{Y} ; Y\right)_{y} \rightarrow 0 .
$$

If $x=(y, z) \in Y \times Z$, the mapping

$$
J_{\infty}\left(T_{Z} ; Z\right)_{z} \otimes F_{y} \rightarrow J_{\infty}(V)_{x},
$$

sending $j_{\infty}(\eta)(z) \otimes j_{\infty}(f)(y)$ into $j_{\infty}\left(\rho^{*} f \cdot \operatorname{pr}_{2}^{-1} \eta\right)(x)$, where $f$ is a real-valued function defined on a neighborhood of $y$ and $\eta \in \mathcal{T}_{Z, z}$, determines an isomorphism

$$
\lambda: J_{\infty}\left(T_{Z} ; Z\right)_{z} \hat{\otimes} F_{y} \rightarrow J_{\infty}(V)_{x}
$$

of linearly compact Lie algebras, which by restriction induces an isomorphism

$$
\lambda: J_{\infty}^{0}\left(T_{Z} ; Z\right)_{z} \hat{\otimes} F_{y}+J_{\infty}\left(T_{Z} ; Z\right)_{z} \hat{\otimes} F_{y}^{0} \rightarrow J_{\infty}^{0}(V)_{x} .
$$

If $x=(y, z)$, the mapping

$$
\lambda:\left(J_{\infty}\left(T_{Z} ; Z\right)_{z} \hat{\otimes} F_{y}\right) \oplus J_{\infty}\left(T_{Y} ; Y\right)_{y} \rightarrow J_{\infty}(T ; \rho)_{x},
$$


sending $(\xi, \eta)$ into $\lambda \xi+\sigma_{x} \eta$, is an isomorphism of transitive Lie algebras between the semi-direct product of $J_{\infty}\left(T_{Y} ; Y\right)_{y}$ and the $J_{\infty}\left(T_{Y} ; Y\right)_{y}$-module $J_{\infty}\left(T_{Z} ; Z\right)_{z} \hat{\otimes} F_{y}$ and $J_{\infty}(T ; \rho)_{x}$; this mapping induces the isomorphism (21.3) and the isomorphism

$$
\lambda:\left(J_{\infty}^{0}\left(T_{Z} ; Z\right)_{z} \hat{\otimes} F_{y}+J_{\infty}\left(T_{Z} ; Z\right)_{z} \hat{\otimes} F_{y}^{0}\right) \oplus J_{\infty}^{0}\left(T_{Y} ; Y\right)_{y} \rightarrow J_{\infty}^{0}(T ; \rho)_{x}
$$

of fundamental subalgebras. Therefore the mapping

$$
J_{\infty}\left(T_{Y} ; Y\right)_{y} \otimes J_{\infty}(V)_{x} \rightarrow J_{\infty}(V)_{x},
$$

sending $\xi \otimes \eta$ into $\left[\sigma_{x} \xi, \eta\right]$, endows $J_{\infty}(V)_{x}$ with the structure of a linearly compact $J_{\infty}\left(T_{Y} ; Y\right)_{y}$-module, and the mapping (21.3) is an isomorphism of $J_{\infty}\left(T_{Y} ; Y\right)_{y}$-modules. If the dimension of $Z$ is $\geqslant 1$, then $J_{\infty}\left(T_{Z} ; Z\right)_{z}$ is a non-abelian and simple Lie algebra, and so $J_{\infty}\left(T_{Z} ; Z\right)_{z} \hat{\otimes} F_{y}$ and $J_{\infty}(V)_{x}$ are non-abelian minimal closed ideals of the semi-direct product and $J_{\infty}(T ; \rho)_{x}$ respectively; moreover

$$
\begin{gathered}
\left(J_{\infty}\left(T_{Z} ; Z\right)_{z} \hat{\otimes} F_{y}\right) \oplus J_{\infty}\left(T_{Y} ; Y\right)_{y} \supset J_{\infty}\left(T_{Z} ; Z\right)_{z} \hat{\otimes} F_{y} \supset 0 \\
J_{\infty}(T ; \rho)_{x} \supset J_{\infty}(V)_{x} \supset 0
\end{gathered}
$$

are Jordan-Hölder sequences for the semi-direct product and $J_{\infty}(T ; \rho)_{x}$ respectively. The mapping

$$
J_{\infty}\left(T_{Z} ; Z\right)_{z} \otimes F_{y} \rightarrow T_{Z, z},
$$

sending $j_{\infty}(\eta)(z) \otimes j_{\infty}(f)(y)$ into $f(y) \eta(z)$, determines a surjective mapping

$$
\varpi: J_{\infty}\left(T_{Z} ; Z\right)_{z} \hat{\otimes} F_{y} \rightarrow T_{Z, z} ;
$$

it is easily seen that the diagram

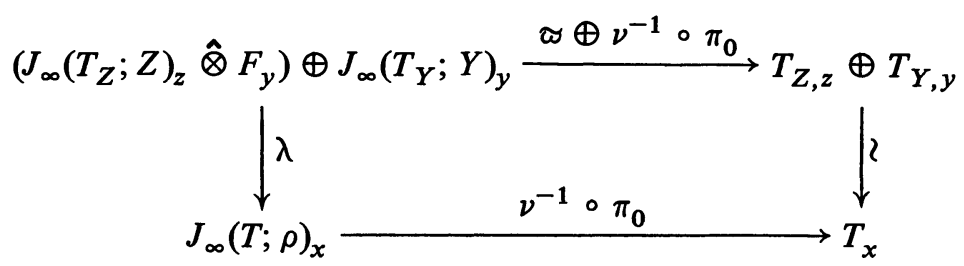

is commutative.

Let $L$ be a transitive subalgebra of $J_{\infty}\left(T_{Z} ; Z\right)_{z}$ and $I$ a closed ideal of $L$. If $L^{0}$ is the fundamental subalgebra $L \cap J_{\infty}^{0}\left(T_{Z} ; Z\right)_{z}$ of $L$, then

$$
M=\left(L \hat{\otimes} F_{y}\right) \oplus J_{\infty}\left(T_{Y} ; Y\right)_{y}
$$

is a transitive Lie algebra, $I \hat{\otimes} F_{y}$ is a closed ideal of $M$ and

$$
M^{0}=\left(L^{0} \hat{\otimes} F_{y}+L \hat{\otimes} F_{y}^{0}\right) \oplus J_{\infty}^{0}\left(T_{Y} ; Y\right)_{y}
$$


is a fundamental subalgebra of $M$. The image of $M$ under the isomorphism (21.4) is a transitive subalgebra of $J_{\infty}(T ; \rho)_{x}$ and the image of the closed ideal $I \hat{\otimes} F_{y}$ of $M$ is contained in $J_{\infty}(V)_{x}$; moreover

$$
\lambda\left(M^{0}\right)=J_{\infty}^{0}(T ; \rho)_{x} \cap \lambda(M) .
$$

By restriction, the mapping $\approx$ induces a surjective mapping

$$
\varpi: L \hat{\otimes} F_{y} \rightarrow T_{Z, z},
$$

and so we obtain an injective mapping

$$
\begin{aligned}
\left(\varpi^{*} \oplus \pi_{0}^{*} \circ \nu^{*-1}\right) \otimes \mathrm{id}:( & \left.T_{Z, z}^{*} \oplus T_{Y, y}^{*}\right) \otimes \mathbf{C} \\
& \rightarrow\left(\left(L \hat{\otimes} F_{y}\right) * \oplus J_{\infty}\left(T_{Y} ; Y\right)_{y}^{*}\right) \otimes \mathbf{C} .
\end{aligned}
$$

The characteristic variety $\mathscr{V}\left(M, I \hat{\otimes} F_{y}, \mathrm{C}\right)$ of the closed ideal $I \hat{\otimes} F_{y}$ of $M$ over $\mathbf{C}$ is a subvariety of the image of (21.7). Since the characteristic variety $\mathcal{V}_{x}\left(J_{1}(V), \mathrm{C}\right)$ of the Lie equation $J_{1}(V)$ is equal to $T_{x}^{*} \otimes \mathrm{C}$, by Theorem 16.4 (i) the characteristic variety $\mathscr{V}_{x}\left(J_{\infty}(T ; \rho)_{x}, J_{\infty}(V)_{x}, \mathbf{G}\right)$ is equal to the image of the injective mapping

$$
\pi_{0}^{*} \circ \nu^{*-1} \otimes \mathrm{id}: T_{x}^{*} \otimes \mathbf{C} \rightarrow J_{\infty}(T ; \rho)_{x}^{*} \otimes \mathbf{C} .
$$

Therefore if $L=J_{\infty}\left(T_{Z} ; Z\right)_{z}$, from the commutativity of diagram (21.6) we deduce that the characteristic variety $\mathscr{V}\left(M, L \hat{\otimes} F_{y}, \mathrm{C}\right)$ of the closed ideal $L \hat{\otimes} F_{y}$ of $M$ is equal to the image of the mapping (21.7).

We no longer assume that $X$ is the product $Y \times Z$, but only suppose that $\rho: X \rightarrow Y$ is a fibered manifold. Let $x \in X$ with $y=\rho(x)$ and let $Z$ be the submanifold $\rho^{-1}(y)$ of $X$; we denote by $z$ the point $x$ considered as a point of $Z$. Then there is a local isomorphism $\varphi: Y \times Z \rightarrow X$ of fibered manifolds over $Y$ defined on a neighborhood of $(y, z)$ such that $\varphi(y, z)=x$. The mapping $\lambda$ defined above together with the isomorphism $\varphi$ gives us an isomorphism of transitive Lie algebras (21.4), which by restriction induces an isomorphism (21.3), and a commutative diagram (21.6). Thus whenever the rank of $V$ is $\geqslant 1$, we see that $(21.5)$ is a Jordan-Hölder sequence for the transitive Lie algebra $J_{\infty}(T ; \rho)_{x}$.

In $\S 26$, we shall require the following result which is the realization theorem of Guillemin-Sternberg [24, Theorem III]:

Proposition 21.1. Let $L$ be a linearly compact real Lie algebra and $N$ an open subalgebra of $L$. Let $Y$ be a manifold whose dimension is equal to that of $L / N$, and $y \in Y$. Then there exists a morphism of pairs of topological Lie algebras

$$
\phi:(L, N) \rightarrow\left(J_{\infty}\left(T_{Y} ; Y\right)_{y}, J_{\infty}^{0}\left(T_{Y} ; Y\right)_{y}\right)
$$

such that $\phi(L)$ is a transitive subalgebra of $J_{\infty}\left(T_{Y} ; Y\right)_{y}$. 
Such a morphism $\phi$ is called a transitive representation of $(L, N)$ on $J_{\infty}\left(T_{Y} ; Y\right)_{y}$. It is easily verified that the kernel of $\phi$ is the largest ideal $D_{L}^{\infty} N$ of $L$ contained in $N$; in particular if $I$ is an ideal contained in $N$, then $\phi$ maps $I$ into zero.

\section{A class of Lie equations determined by forms}

We continue to assume that $\rho: X \rightarrow Y$ is a surjective submersion. Let $E$ be a fibered manifold over $Y$ and $E^{\prime}$ be the fibered manifold $\rho^{-1} E$ over $X$. If $e^{\prime}=(x, e) \in E^{\prime}$, with $x \in X, e \in E$, then $V_{e^{\prime}}\left(E^{\prime}\right)$ is equal to $T_{e}(E / Y)$. Let

$$
\rho^{*}: \rho^{-1} S^{k} T_{Y}^{*} \rightarrow S^{k} T^{*}
$$

be the monomorphism of vector bundles over $X$, whose restriction to the fiber over $x \in X$ is induced by the mapping

$$
\rho_{x}^{*}: T_{Y, \rho(x)}^{*} \rightarrow T_{x}^{*},
$$

and denote its image by $\rho^{*}\left(S^{k} T_{Y}^{*}\right)$. Let

$$
\rho_{k}: \rho^{-1} J_{k}(E ; Y) \rightarrow J_{k}\left(E^{\prime}\right)
$$

be the morphism of fibered manifolds over $X$ sending $\left(x, j_{k}(s)(y)\right)$ into $j_{k}(s \circ \rho)(x)$, with $x \in X, s \in \mathcal{E}_{y}$ and $\rho(x)=y$.

Proposition 22.1. The morphism $\rho_{k}$ is a monomorphism of affine bundles over $\rho_{k-1}$ whose associated morphism of vector bundles is induced by the monomorphism of vector bundles

$$
\rho^{*} \otimes \mathrm{id}: S^{k} T_{Y}^{*} \otimes_{E^{\prime}} T(E / Y) \rightarrow S^{k} T^{*} \otimes_{E^{\prime}} V\left(E^{\prime}\right)
$$

over $E^{\prime}$.

Proof. Let $f$ be a real-valued function on $Y$ satisfying $j_{k-1}(f)(y)=0$, with $y \in Y$. If $u$ is the unique element of $S^{k} T_{Y, y}^{*}$ satisfying $\varepsilon u=j_{k}(f)(y)$, then for $x \in X$, with $\rho(x)=y$, the element $\rho_{x}^{*} u$ is determined by the relation

$$
\varepsilon\left(\rho_{x}^{*} u\right)=j_{k}(f \circ \rho)(x)
$$

To prove the proposition, it suffices to show that, if $s$ is a section of $E$ over a neighborhood of $y$ and $\xi \in T_{s(y)}(E / Y)$, we have

$$
\rho_{k}\left(x, j_{k}(s)(y)+u \otimes \xi\right)=j_{k}(s \circ \rho)(x)+\rho_{x}^{*} u \otimes \xi,
$$

where $\xi$ on the right-hand side is considered as an element of $V_{(x, s(y))}\left(E^{\prime}\right)$. In fact, let $\tilde{s}$ be a deformation of the section $s$, that is, a mapping $\tilde{s}: U \times(-\varepsilon, \varepsilon) \rightarrow$ $E$, where $U$ is a neighborhood of $y$ satisfying $\tilde{s}(b, 0)=s(b)$ and $s(b, t) \in E_{b}$ for $b \in U, t \in(-\varepsilon, \varepsilon)$, such that

$$
\left.\frac{d \tilde{s}(y, t)}{d t}\right|_{t=0}=\xi .
$$


Then the mapping $\tilde{s}^{\prime}: \rho^{-1}(U) \times(-\varepsilon, \varepsilon) \rightarrow E$ sending $(a, t)$ into $\tilde{s}(\rho(a), t)$ is a deformation of the section $s \circ \rho$ over $\rho^{-1}(U)$ satisfying

$$
\left.\frac{d \tilde{s}^{\prime}(x, t)}{d t}\right|_{t=0}=\xi
$$

considered as an element of $V_{(x, s(y))}\left(E^{\prime}\right)$. According to [9, Lemma 5.1 and Proposition 5.1], we know that

$$
\begin{gathered}
j_{k}(s)(y)+u \otimes \xi=j_{k}(\sigma)(y), \\
j_{k}(s \circ \rho)(x)+\rho_{x}^{*} u \otimes \xi=j_{k}\left(\sigma^{\prime}\right)(x),
\end{gathered}
$$

where $\sigma$ is the section

$$
b \mapsto \tilde{s}(b, f(b))
$$

of $E$ over a neighborhood of $y$, and $\sigma^{\prime}$ is the section

$$
a \mapsto \tilde{s}^{\prime}(a,(f \circ \rho)(a))
$$

of $E^{\prime}$ over a neighborhood of $x$. Since $\sigma^{\prime}=\sigma \circ \rho$, we see that

$$
j_{k}\left(\sigma^{\prime}\right)(x)=\rho_{k}\left(x, j_{k}(\sigma)(y)\right),
$$

and so (22.1) follows from (22.2), (22.3) and (22.4).

Now assume that $E$ and $E^{\prime}$ are vector bundles over $X$. If $\varphi: E \rightarrow E^{\prime}$ is a morphism of vector bundles, we denote by

$$
J_{k}(\varphi): J_{k}(E) \rightarrow J_{k}\left(E^{\prime}\right)
$$

the unique morphism of vector bundles sending $j_{k}(s)(x)$ into $j_{k}(\varphi s)(x)$, where $s$ is a section of $E$ over a neighborhood of $x$. If $P: \mathcal{E} \rightarrow \mathcal{E}^{\prime}$ is a differential operator of order $k$, let

$$
p_{l}(P): J_{k+l}(E) \rightarrow J_{l}\left(E^{\prime}\right)
$$

be the morphism of vector bundles sending $j_{k+l}(s)(x)$ into $j_{l}(P s)(x)$, where $s$ is a section of $E$ over a neighborhood of $x$.

For the remainder of this section, assume that $E$ is a vector bundle over $Y$. The sequence

$$
0 \rightarrow \rho^{-1} \mathcal{E} \longrightarrow \mathcal{E}_{X} \stackrel{d_{X / Y}}{\longrightarrow} \mathcal{\Upsilon}^{*} \otimes \mathcal{E}_{X} \stackrel{d_{X / Y}}{\longrightarrow} \wedge^{2} \mathfrak{V}^{*} \otimes \mathcal{E}_{X} \stackrel{d_{X / Y}}{\longrightarrow}
$$

$$
\cdots \rightarrow \wedge^{q} \mathscr{\Upsilon}^{*} \otimes \mathcal{E}_{X} \rightarrow 0
$$


is exact, where $q=\operatorname{rank} V$, and formally exact in the sense that the sequences

$$
\begin{gathered}
0 \longrightarrow \rho^{-1} J_{k}(E ; Y) \stackrel{\rho_{k}}{\longrightarrow} J_{k}\left(\rho^{-1} E\right) \stackrel{p_{k-1}\left(d_{X / Y}\right)}{\longrightarrow} J_{k-1}\left(V^{*} \otimes_{X} E\right) \stackrel{p_{k-2}\left(d_{X / Y}\right)}{\longrightarrow} \\
J_{k-2}\left(\bigwedge^{2} V^{*} \otimes_{X} E\right) \stackrel{p_{k-3}\left(d_{X / Y}\right)}{\longrightarrow} \cdots \rightarrow J_{k-q}\left(\bigwedge^{q} V^{*} \otimes_{X} E\right) \rightarrow 0
\end{gathered}
$$

are exact for $k \geqslant 0$. Indeed, it is easily seen that the sub-bundle $\rho^{*} T_{Y}^{*} \otimes_{X} E$ of $T^{*} \otimes_{X} E$, corresponding to the differential equation $\rho_{1}\left(\rho^{-1} J_{1}(E ; Y)\right) \subset J_{1}\left(\rho^{-1} E\right)$ on $\rho^{-1} E$, is involutive. Moreover the sequences

$$
\begin{aligned}
& 0 \rightarrow \rho^{*}\left(S^{k} T_{Y}^{*}\right) \otimes_{X} E \rightarrow S^{k} T^{*} \otimes_{X} E \rightarrow S^{k-1} T^{*} \otimes V^{*} \otimes_{X} E \rightarrow \cdots \\
& \rightarrow S^{2} T^{*} \otimes \wedge^{k-2} V^{*} \otimes_{X} E \rightarrow T^{*} \otimes \wedge^{k-1} V^{*} \otimes_{X} E \rightarrow \wedge^{k} V^{*} \otimes_{X} E \rightarrow 0,
\end{aligned}
$$

whose mappings are induced by the mappings $p_{l}\left(d_{X / Y}\right)$, are exact and (22.5) is the Spencer sequence of this formally integrable first-order equation on $\rho^{-1} E$ constructed according to the method of [8, Theorem 5.1]. Furthermore, if $R_{1} \subset J_{1}\left(\wedge^{r} V^{*} \otimes_{X} E\right)$, with $r \geqslant 1$, is the differential equation equal to the kernel of $p_{0}\left(d_{X / Y}\right)$, from the involutivity of $\rho^{*} T_{Y}^{*} \otimes_{X} E$ and the exactness of the sequences (22.7) and (22.6), we deduce by [8, Proposition 4.3] (see also the proof of [3, Theorem 4.4]) that $g_{1}$ is involutive and that $R_{1}$ is formally integrable, with $\pi_{0} R_{1}=\wedge^{r} V^{*} \otimes_{X} E$. According to [10, Theorem 3], the exactness of the sequences (22.5) and (22.6) implies that

$$
H^{j}\left(\rho_{1}\left(\rho^{-1} J_{1}(E ; Y)\right)\right)=0, \quad H^{j}\left(R_{1}\right)=0, \quad \text { for } j>0 .
$$

By Proposition 16.4, for $x \in X$, the characteristic variety $\mathcal{V}_{x}\left(\rho_{1}\left(\rho^{-1} J_{1}(E ; Y)\right), \mathrm{C}\right)$ is equal to the subspace $\rho_{x}^{*} T_{Y, \rho(x)}^{*} \otimes \mathbf{C}$ of $T_{x}^{*} \otimes \mathbf{C}$. From the exactness of the sequences (22.7), we see that the characteristic variety $\mathscr{V}_{x}\left(R_{1}, \mathrm{C}\right)$ is equal to $T_{x}^{*} \otimes \mathbf{C}$.

Next we recall the following formulas. If $u \in \mathcal{T}^{*} \otimes J_{1}(\mathscr{T})$ and $u_{0}=\pi_{0} u$, $\tilde{u}_{0}=\nu^{-1} \circ u_{0}$, then, for $\xi, \eta \in \mathcal{T}$, we have the identity

$$
\begin{aligned}
\left\langle\xi \wedge \eta, \mathfrak{D}_{1} u\right\rangle= & \nu\left[\left(\mathrm{id}+\tilde{u}_{0}\right) \xi,\left(\mathrm{id}+\tilde{u}_{0}\right) \eta\right]-\left(\nu+u_{0}\right)[\xi, \eta] \\
& -\mathcal{L}\left(\nu^{-1} u(\xi)\right)\left(\left(\nu+u_{0}\right) \eta\right)+\mathcal{L}\left(\nu^{-1} u(\eta)\right)\left(\left(\nu+u_{0}\right) \xi\right),
\end{aligned}
$$

which is given by [18, Lemma 3.1] and which is equivalent to the identity

$$
\begin{aligned}
\tilde{u}_{0}[\xi, \eta]= & {\left[\tilde{u}_{0}(\xi), \tilde{u}_{0}(\eta)\right]+\left[\tilde{u}_{0}(\xi), \eta\right]+\left[\xi, \tilde{u}_{0}(\eta)\right] } \\
& -\nu^{-1} \mathcal{L}\left(\nu^{-1} u(\xi)\right)\left(\left(\nu+u_{0}\right) \eta\right) \\
& +\nu^{-1} \mathcal{L}\left(\nu^{-1} u(\eta)\right)\left(\left(\nu+u_{0}\right) \xi\right)-\nu^{-1}\left\langle\xi \wedge \eta, \mathfrak{D}_{1} u\right\rangle .
\end{aligned}
$$


The relation (22.9) is analogous to the formula for the operator $\overline{\mathfrak{D}}_{1}$ on $J_{0}(\mathcal{T})^{*} \otimes \tilde{J}_{1}(\mathcal{T})$ of Lemma 1.3:

$$
\begin{aligned}
\left\langle\xi \wedge \eta, \overline{\mathfrak{D}}_{1} u\right\rangle= & -\left[\left(\mathrm{id}-\tilde{u}_{0}\right) \tilde{\xi},\left(\mathrm{id}-\tilde{u}_{0}\right) \tilde{\eta}\right]+\left(\mathrm{id}-\tilde{u}_{0}\right)[\tilde{\xi}, \tilde{\eta}] \\
& -\left(\nu^{-1}-u_{0}\right)\{\mathcal{L}(u(\xi)) \eta-\mathcal{L}(u(\eta)) \xi\},
\end{aligned}
$$

where $u \in J_{0}(\mathcal{T})^{*} \otimes \tilde{J}_{1}(\mathcal{T}), u_{0}=\pi_{0} u, \tilde{u}_{0}=u_{0} \circ \nu$ and $\xi, \eta \in J_{0}(\mathcal{T}), \tilde{\xi}=\nu^{-1} \xi$, $\tilde{\eta}=\nu^{-1} \eta$.

If $u \in T^{*} \otimes J_{0}(T)$, we set $\tilde{u}=\nu^{-1} \circ u \in T^{*} \otimes T$. The mapping sending $\alpha \in \wedge T^{*}$ into the element

$$
\alpha^{u}=(\mathrm{id}+\tilde{u})^{*} \alpha=\alpha \circ(\mathrm{id}+\tilde{u})
$$

of $\wedge T^{*}$, where id is the identity mapping of $T$, is an endomorphism of the exterior algebra $\wedge T^{*}$. If $u \in\left(T^{*} \otimes J_{0}(T)\right)^{\wedge}$, it is an isomorphism; if $u=0$, it is the identity mapping. If $\alpha \in \wedge^{0} T^{*}$, then $\alpha^{u}=\alpha$. We also set $\alpha^{u}=$ $\nu^{*-1}\left(\nu^{*} \alpha\right)^{u}$, for $\alpha \in \wedge J_{0}(T)^{*}$. For $v=\beta \otimes \eta \in \wedge T^{*} \otimes T$ and $\alpha \in \wedge T^{*}$, we define the element

$$
v \pi_{u} \alpha=\beta \wedge(i(\eta) \alpha)^{u}
$$

of $\wedge T^{*}$ and extend this operation to arbitrary $v$ by linearity. Then

$$
v \pi_{u}(\alpha \wedge \beta)=\left(v \pi_{u} \alpha\right) \wedge \beta^{u}+(-1)^{p q} \alpha^{u} \wedge\left(v \pi_{u} \beta\right)
$$

for all $v \in \wedge{ }^{p} T^{*} \otimes T, \alpha \in \wedge{ }^{q} T^{*}, \beta \in \wedge T^{*}$. Next, if $u$ is a section of $T^{*} \otimes J_{0}(T)$, for $v=\beta \otimes \tilde{\eta} \in \wedge J_{0}(\mathcal{T})^{*} \otimes \tilde{J}_{1}(\mathcal{T}), \alpha \in \wedge J_{0}(\mathcal{T})^{*}$, we define the element

$$
\varrho_{u}(v) \alpha=\beta \wedge(\varrho(\tilde{\eta}) \alpha)^{u}
$$

of $\wedge J_{0}(\mathcal{T})^{*} \otimes \tilde{J}_{1}(\mathcal{T})$ and extend this operation to arbitrary $v$ by linearity. Then

$$
\bigodot_{u}(v)(\alpha \wedge \beta)=\left(\complement_{u}(v) \alpha\right) \wedge \beta^{u}+(-1)^{p q} \alpha^{u} \wedge\left(\complement_{u}(v) \beta\right)
$$

for all $v \in \wedge{ }^{p} J_{0}(\mathcal{T})^{*} \otimes \tilde{J}_{1}(\mathcal{T}), \alpha \in \wedge{ }^{q} J_{0}(\mathcal{T})^{*}, \beta \in \wedge J_{0}(\mathcal{T})^{*}$; moreover if $v \in J_{0}(\mathcal{T})^{*} \otimes \tilde{J}_{1}(\mathcal{T}), \alpha \in J_{0}(\mathcal{T})^{*}$, then

$$
\begin{aligned}
\left\langle\xi \wedge \eta, \mathscr{L}_{u}(v) \alpha\right\rangle= & \left\langle\left(\operatorname{id}+u \circ \nu^{-1}\right) \eta, \mathscr{L}(v(\xi)) \alpha\right\rangle \\
& -\left\langle\left(\operatorname{id}+u \circ \nu^{-1}\right) \xi, \mathcal{L}(v(\eta)) \alpha\right\rangle,
\end{aligned}
$$

for $\xi, \eta \in J_{0}(\sigma)$.

If $u$ is a section of $T^{*} \otimes J_{0}(T)$ and $\omega$ is a form on $X$, then the following proposition gives us the fundamental formula (22.15) relating $d \omega^{u}$ and $(d \omega)^{u}$. 
Proposition 22.2. Let $\omega$ be a section of $\wedge T^{*}$ and $u$ a section of $T^{*} \otimes J_{1}(T)$ over $X$. Then

$$
d \omega^{u_{0}}-(d \omega)^{u_{0}}=\left(\nu^{-1} \circ \mathfrak{D}_{1} u\right) \pi_{u_{0}} \omega-\nu^{*} \mathcal{L}_{u_{0}}(\hat{u})\left(\nu^{*-1} \omega\right),
$$

where $u_{0}=\pi_{0} u$ and $\hat{u}$ is the section $\left(\nu^{*-1} \otimes \nu^{-1}\right) u$ of $J_{0}(T)^{*} \otimes \tilde{J}_{1}(T)$.

Proof. Let $u$ be a section of $T^{*} \otimes J_{1}(T)$ over $X$; we set $\tilde{u}_{0}=\nu^{-1} \circ u_{0}$. Since $d$ is a derivation of $\wedge \mathcal{T}^{*}$ of degree 1 and the relations (22.12) and (22.13) hold, it suffices to verify (22.15) when $\omega$ is a real-valued function $f$ or a 1-form on $X$. In the first case, for $\xi \in \mathcal{T}$ we have

$$
\begin{aligned}
\left\langle\xi, d f-(d f)^{u_{0}}\right\rangle & =\xi \cdot f-\left[\left(\mathrm{id}+\tilde{u}_{0}\right) \xi\right] \cdot f=-\tilde{u}_{0}(\xi) \cdot f \\
& =-\left\langle\xi, \nu^{*} \mathcal{L}(\hat{u}) f\right\rangle=-\left\langle\xi, \nu^{*} \mathcal{L}_{u_{0}}(\hat{u}) f\right\rangle,
\end{aligned}
$$

which reduces to (22.15) with $\omega=f$. Next, suppose that $\omega$ is a 1 -form on $X$; for $\xi, \eta \in \mathcal{T}$, we have by (22.10) and (22.14)

$$
\begin{aligned}
\left\langle\xi \wedge \eta, d \omega^{u_{0}}\right\rangle= & \xi \cdot\left\langle\eta, \omega^{u_{0}}\right\rangle-\eta \cdot\left\langle\xi, \omega^{u_{0}}\right\rangle-\left\langle[\xi, \eta], \omega^{u_{0}}\right\rangle \\
= & \xi \cdot\langle\eta, \omega\rangle-\eta \cdot\langle\xi, \omega\rangle-\langle[\xi, \eta], \omega\rangle \\
& +\xi \cdot\left\langle\tilde{u}_{0}(\eta), \omega\right\rangle-\eta \cdot\left\langle\tilde{u}_{0}(\xi), \omega\right\rangle-\left\langle\tilde{u}_{0}[\xi, \eta], \omega\right\rangle \\
= & \langle\xi \wedge \eta, d \omega\rangle+\xi \cdot\left\langle\tilde{u}_{0}(\eta), \omega\right\rangle-\eta \cdot\left\langle\tilde{u}_{0}(\xi), \omega\right\rangle \\
& -\left\langle\left[\tilde{u}_{0}(\xi), \tilde{u}_{0}(\eta)\right], \omega\right\rangle-\left\langle\left[\tilde{u}_{0}(\xi), \eta\right], \omega\right\rangle \\
& -\left\langle\left[\xi, \tilde{u}_{0}(\eta)\right], \omega\right\rangle+\left\langle\nu^{-1} \mathcal{L}\left(\nu^{-1} u(\xi)\right)\left(\left(\nu+u_{0}\right) \eta\right), \omega\right\rangle \\
& -\left\langle\nu^{-1} \mathcal{L}\left(\nu^{-1} u(\eta)\right)\left(\left(\nu+u_{0}\right) \xi\right), \omega\right\rangle \\
& +\left\langle\nu^{-1}\langle\xi \wedge \eta, \mathfrak{D} 1 u\rangle, \omega\right\rangle \\
= & \langle\xi \wedge \eta, d \omega\rangle+\xi \cdot\left\langle\tilde{u}_{0}(\eta), \omega\right\rangle-\eta \cdot\left\langle\tilde{u}_{0}(\xi), \omega\right\rangle \\
& -\left\langle\left[\tilde{u}_{0}(\xi), \tilde{u}_{0}(\eta)\right], \omega\right\rangle-\left\langle\left[\tilde{u}_{0}(\xi), \eta\right], \omega\right\rangle \\
& -\left\langle\left[\xi, \tilde{u}_{0}(\eta)\right], \omega\right\rangle+\tilde{u}_{0}(\xi) \cdot\left\langle\left(\mathrm{id}+\tilde{u}_{0}\right) \eta, \omega\right\rangle \\
& -\tilde{u}_{0}(\eta) \cdot\left\langle\left(\mathrm{id}+\tilde{u}_{0}\right) \xi, \omega\right\rangle-\left\langle\left(\nu+u_{0}\right) \eta, \mathcal{L}\left(\nu^{-1} u(\xi)\right)\left(\nu^{*-1} \omega\right)\right\rangle \\
& +\left\langle\left(\nu+u_{0}\right) \xi, \mathcal{L}\left(\nu^{-1} u(\eta)\right)\left(\nu^{*-1} \omega\right)\right\rangle \\
& +\left\langle\xi \wedge \eta,\left(\nu^{-1} \circ \mathfrak{D}_{1} u\right) \pi \omega\right\rangle \\
= & \left\langle\xi \wedge \eta,(d \omega)^{u_{0}}+\left(\nu^{-1} \circ \mathfrak{D}{ }_{1} u\right) \pi \omega-\nu^{*} \mathcal{L}_{u_{0}}(\hat{u})\left(\nu^{*-1} \omega\right)\right\rangle,
\end{aligned}
$$

from which we deduce (22.15) when $\omega$ is a section of $T^{*}$ and hence also in the general case.

Let $f: X \rightarrow X$ be a local diffeomor phism defined on an open set $U$. If $\xi$ is a vector field on $U$, we define the vector field $f_{*} \xi$ on $f(U)$ by

$$
\left(f_{*} \xi\right)(x)=f_{*}\left(\xi\left(f^{-1}(x)\right)\right)
$$


for $x \in U$. If $\omega$ is a section of $\wedge T^{*}$ over $X$, it is easily seen that

$$
\varrho(\xi) f^{*} \omega=f^{*} \varrho\left(f_{*} \xi\right) \omega .
$$

If $\xi$ is a $\rho$-projectable section of $T$ over an open set $U \subset X$ and $\eta \in \wedge \mathscr{V}$, then $\mathcal{L}(\xi) \eta \in \wedge \mathcal{V}$; if $\omega$ is a section of $\wedge V^{*}$, then $\mathcal{L}(\xi) \omega$ is the section of $\wedge V^{*}$ satisfying

$$
\langle\eta, \mathcal{L}(\xi) \omega\rangle=\mathscr{L}(\xi)\langle\eta, \omega\rangle-\langle\mathcal{L}(\xi) \eta, \omega\rangle,
$$

for all $\eta \in \wedge \mathcal{V}$. If $f: X \rightarrow X$ is a $\rho$-projectable local diffeomorphism defined on $U$, then $f_{*} \xi$ is a $\rho$-projectable vector field on $f(U)$ and formula (22.16) holds. If $g$ is a real-valued function on $Y$, then

$$
\mathcal{L}((g \circ \rho) \xi) \omega=(g \circ \rho) \mathcal{L}(\xi) \omega .
$$

If $\xi$ is section of $V$, then

$$
\mathcal{L}(\xi) \omega=d_{X / Y}(\xi \pi \omega)+\xi \pi d_{X / Y} \omega,
$$

and so

$$
\mathcal{L}(\xi) d_{X / Y} \omega=d_{X / Y} \mathcal{L}(\xi) \omega ;
$$

if $\omega$ is a section of $\wedge V^{*} \otimes_{X} E$, formula (22.17) or (22.18) defines a section $\mathcal{L}(\xi) \omega$ of $\wedge V^{*} \otimes_{X} E$. If $\tilde{\xi} \in \tilde{J}_{1}(T ; \rho)$ and $\alpha \in \wedge J_{0}(\mathscr{V})^{*}$, then $\mathcal{L}(\tilde{\xi}) \alpha \in$ $\wedge J_{0}(V)^{*}$ is determined by

$$
\langle\eta, \mathcal{L}(\tilde{\xi}) \alpha\rangle=\mathscr{L}(\tilde{\xi})\langle\eta, \alpha\rangle-\langle\mathscr{L}(\tilde{\xi}) \eta, \alpha\rangle
$$

for all $\eta \in \wedge J_{0}(\mathscr{V})$. Moreover if $\tilde{\xi} \in \tilde{J}_{1}(V), \alpha \in \wedge J_{0}(\mathscr{V})^{*} \otimes \mathcal{E}_{X}$, formula (22.19) defines $\mathcal{L}(\tilde{\xi}) \alpha \in \wedge J_{0}(V)^{*} \otimes_{X} E$.

If $u$ is a section of $V^{*} \otimes J_{0}(V)$, the operations depending on $u$ described above are easily modified to define elements $\alpha^{u}, v \pi_{u} \alpha$ of $\wedge V^{*} \otimes_{X} E$, for $\alpha \in \wedge V^{*} \otimes_{X} E, v \in \wedge V^{*} \otimes V$, and $\mathcal{L}_{u}(v) \alpha$ of $\wedge J_{0}(\mathcal{V})^{*} \otimes \mathcal{E}_{X}$, for $v \in$ $\wedge J_{0}(\mathscr{V})^{*} \otimes \tilde{J}_{1}(\mathscr{V}), \quad \alpha \in \wedge J_{0}(\mathscr{V})^{*} \otimes \mathcal{E}_{X}$. If $u \in T^{*} \otimes J_{0}(V)$ and $\alpha \in$ $\wedge V^{*} \otimes_{X} E$, we set $\alpha^{u}=\alpha^{v}$, where $v=u_{\mid V}$. According to (1.5) and the definition of the bracket on $\wedge T^{*} \otimes J_{1}(T)$, for $u \in \mathcal{T}^{*} \otimes J_{1}(\mathscr{V})$ the element $\mathfrak{D}_{1} u_{\mid \wedge{ }^{2} V}$ of $\wedge \mathfrak{V}^{*} \otimes J_{0}(\mathscr{V})$ depends only on $u_{\mid V}$; we set

$$
\mathfrak{D}_{1, X / Y}\left(u_{\mid V}\right)=\left(\mathfrak{D}_{1} u\right)_{\mid \wedge{ }^{2} V}
$$

and thus obtain an operator

$$
\mathfrak{D}_{1, X / Y}: \mathfrak{V}^{*} \otimes J_{1}(\mathfrak{V}) \rightarrow \wedge^{2 \mathfrak{V} *} \otimes J_{0}(\mathfrak{V})
$$

The following proposition is a generalization of Proposition 22.2 and gives us the fundamental formula (22.20) relating $d_{X / Y} \omega^{u}$ and $\left(d_{X / Y} \omega\right)^{u}$, where $u$ is a section of $T^{*} \otimes J_{0}(V)$ and $\omega$ is a section of $\wedge V^{*} \otimes_{X} E$ over $X$. 
Proposition 22.3. Let $\omega$ be a section of $\wedge V^{*} \otimes_{X} E$ and $u$ a section of $V^{*} \otimes J_{1}(V)$ over $X$. Then

$$
d_{X / Y} \omega^{u_{0}}-\left(d_{X / Y} \omega\right)^{u_{0}}=\left(\nu^{-1} \circ \mathfrak{D}_{1, X / Y} u\right) \pi_{u_{0}} \omega-\nu^{*} \bigodot_{u_{0}}(\hat{u})\left(\nu^{*-1} \omega\right),
$$

as sections of $\wedge V^{*} \otimes_{X} E$, where $u_{0}=\pi_{0} u$ and $\hat{u}$ is the section $\left(\nu^{*-1} \otimes \nu^{-1}\right) u$ of $J_{0}(V) * \otimes \tilde{J}_{1}(V)$.

Proof. If we restrict the forms of both sides of (22.15) to $\wedge V$, we obtain (22.20) when $\omega$ is a section of $\wedge V^{*}$. The proposition is an immediate consequence of this special case.

We now introduce a class of Lie equations determined by forms. Let $\omega$ be a section of $\wedge V^{*} \otimes_{X} E$ over $X$, and let

$$
D_{\omega}: \mathfrak{V} \rightarrow \wedge \mathcal{V}^{*} \otimes \varepsilon_{X}
$$

be the linear differential operator sending $\xi$ into $\mathcal{L}(\xi) \omega$. The kernel $J_{k}(V ; \omega)$ of $p_{k-1}\left(D_{\omega}\right)$ is a sub-bundle of $J_{k}(V)$ with possibly varying fiber. Then $\xi \in J_{1}(V)$ belongs to $J_{1}(V ; \omega)$ if and only if $\varrho(\tilde{\xi}) \nu^{*-1} \omega=0$, where $\tilde{\xi}=\nu^{-1} \xi$. It follows that if $J_{1}(V ; \omega)$ is a vector bundle, then it is a Lie equation whose $k$ th prolongation is $J_{k+1}(V ; \omega)$, and which is the Lie equation determined by the form $\omega$. Let $g_{k}(V ; \omega) \subset S^{k} J_{0}(T)^{*} \otimes J_{0}(V)$ be the kernel of $\pi_{k-1}: J_{k}(V ; \omega) \rightarrow$ $J_{k-1}(V ; \omega)$, where $J_{0}(V ; \omega)=J_{0}(V)$.

In $\$ 23$ and $\S 24$, we shall consider sections $\omega$, and the corresponding Lie equations, satisfying the condition of the following:

Proposition 22.4. Let $\omega$ be a section of $\wedge^{r} V^{*} \otimes_{X} E$ over $X$, with $r \geqslant 1$. If there are isomorphisms of vector bundles

$$
\begin{gathered}
\sigma: V \rightarrow \wedge^{r-1} V^{*} \otimes_{X} E, \\
\tau: \wedge^{r} V^{*} \otimes_{X} E \rightarrow \wedge^{r} V^{*} \otimes_{X} E
\end{gathered}
$$

over $X$ such that the diagram

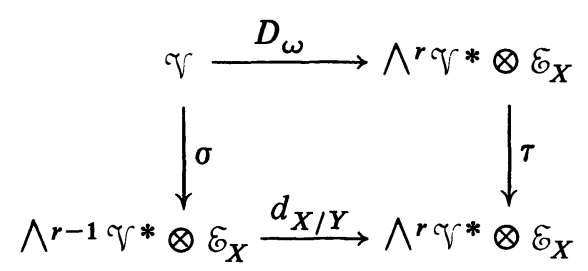

commutes, then $J_{1}(V ; \omega)$ is a formally integrable Lie equation, $g_{1}(V ; \omega)$ is involutive, $\pi_{0}: J_{1}(V ; \omega) \rightarrow J_{0}(V)$ is surjective, and

$$
H^{j}\left(J_{1}(V ; \omega)\right)=0, \text { for } j>0 .
$$

Moreover, for $x \in X$ the characteristic variety of $J_{1}(V ; \omega)$ over $\mathbf{C}$ is equal to $\rho_{x}^{*} T_{Y, \rho(x)} \otimes \mathbf{C}$ if $r=1$, or to $T_{x}^{*} \otimes \mathbf{C}$ if $r>1$. 
Proof. First if $r \geqslant 2$, since $\sigma, \tau$ are isomorphisms and the sequence (22.6) is exact, $J_{k}(V ; \omega)$ is equal to the image of the kernel of

$$
p_{k-1}\left(d_{X / Y}\right): J_{k}\left(\wedge^{r-1} V^{*} \otimes_{X} E\right) \rightarrow J_{k-1}\left(\wedge^{r} V^{*} \otimes_{X} E\right)
$$

under the isomorphism

$$
J_{k}\left(\sigma^{-1}\right): J_{k}\left(\wedge^{r-1} V^{*} \otimes_{X} E\right) \rightarrow J_{k}(V)
$$

induced by $\sigma^{-1}$. Next if $r=1$, the commutative and exact diagram

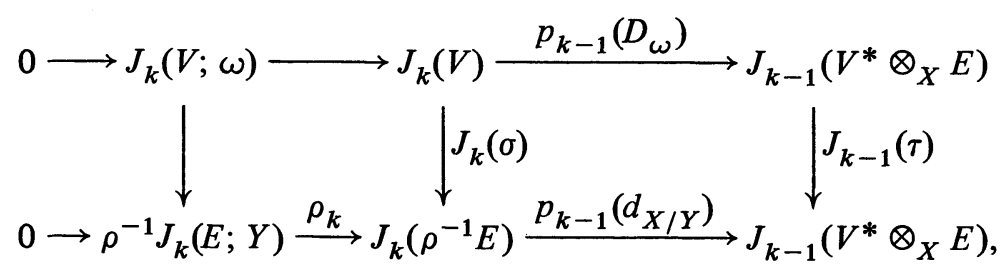

whose mappings $J_{k}(\sigma)$ and $J_{k-1}(\tau)$ are isomorphisms of vector bundles, induces an isomorphism $J_{k}(V ; \omega) \rightarrow \rho^{-1} J_{k}(E ; Y)$. The conclusions of the proposition are direct consequences of our discussion of the sequence (22.5) and the equations $\rho_{1}\left(\rho^{-1} J_{1}(E ; Y)\right)$ and $R_{1}$, and of (22.8).

A local mapping $X \rightarrow X$ over $Y$ is a $\rho$-projectable mapping inducing the identity mapping of $Y$. If $f$ is such a local mapping $X \rightarrow X$ over $Y$, let $\mathfrak{D}_{\omega} f$ denote the section $f^{*} \omega-\omega$ of $\wedge V^{*} \otimes_{X} E$. Let

$$
p_{k-1}\left(\mathfrak{D}_{\omega}\right): Q_{k}(V) \rightarrow J_{k-1}\left(\wedge V^{*} \otimes_{X} E\right)
$$

be the morphism of fibered manifolds over $X$ sending $\phi=j_{k}(f)(a)$ into $j_{k-1}\left(\mathfrak{D}_{\omega} f\right)(a)$, where $f$ is a local diffeomorphism of $X$ over $Y$ defined on a neighborhood of $a \in X$. If target $\phi=b$, we define an isomorphism

$$
\phi: J_{k-1}\left(\wedge V^{*} \otimes_{X} E\right)_{b} \rightarrow J_{k-1}\left(\wedge V^{*} \otimes_{X} E\right)_{a}
$$

by setting

$$
\phi\left(j_{k-1}(u)(b)\right)=j_{k-1}\left(f^{*} u\right)(a),
$$

where $u$ is a section of $\wedge V^{*} \otimes_{X} E$ over $X$; then the diagram

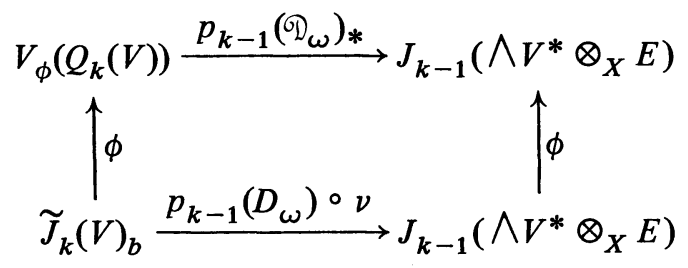


commutes. Indeed, if $g_{t}$ is a one-parameter family of local diffeomorphisms of $X$ over $Y$ defined on a neighborhood $U$ of $b$, with $g_{0}$ the identity mapping of $U$, we set $\psi(t)=j_{k}\left(g_{t}\right)(b)$ and let $\xi$ be the section $d g_{t} /\left.d t\right|_{t=0}$ of $V$ over $U$; then

$$
\tilde{j_{k}}(\xi)(b)=\left.\frac{d}{d t} \psi(t)\right|_{t=0}
$$

and

$$
\begin{aligned}
p_{k-1}\left(\mathfrak{D}_{\omega}\right)_{*} \cdot \phi\left(\tilde{j}_{k}(\xi)(b)\right) & =\left.p_{k-1}\left(\mathfrak{D}_{\omega}\right)_{*} \frac{d}{d t} \psi(t) \cdot \phi\right|_{t=0} \\
& =\left.\frac{d}{d t} p_{k-1}\left(\mathfrak{D}_{\omega}\right)(\psi(t) \cdot \phi)\right|_{t=0} \\
& =\left.\frac{d}{d t} j_{k-1}\left(\left(g_{t} \circ f\right)^{*} \omega-\omega\right)(a)\right|_{t=0} \\
& =\left.\frac{d}{d t} j_{k-1}\left(f^{*} \cdot g_{t}^{*} \omega\right)(a)\right|_{t=0} \\
& =\left.\frac{d}{d t} \phi \cdot j_{k-1}\left(g_{t}^{*} \omega\right)(b)\right|_{t=0} \\
& =\left.\phi \cdot \frac{d}{d t} j_{k-1}\left(g_{t}^{*} \omega\right)(b)\right|_{t=0} \\
& =\phi \cdot j_{k-1}\left(\left.\frac{d}{d t} g_{t}^{*} \omega\right|_{t=0}\right)(b) \\
& =\phi \cdot j_{k-1}(\mathcal{L}(\xi) \omega)(b)=\phi\left(p_{k-1}\left(D_{\omega}\right) j_{k}(\xi)(b)\right) .
\end{aligned}
$$

From the commutativity of (22.23), we see that $J_{k}(V ; \omega)$ is a vector bundle if and only if $p_{k-1}\left(\mathfrak{D}_{\omega}\right)$ has constant rank. Let

$$
J_{k}\left(\mathfrak{D}_{\omega}\right): J_{k}\left(Q_{1}(V)\right) \rightarrow J_{k}\left(\wedge V^{*} \otimes_{X} E\right)
$$

be the morphism of fibered manifolds sending $j_{k}(\phi)(a)$ into $j_{k}\left(p_{0}\left(\mathfrak{D}_{\omega}\right) \phi\right)(a)$, where $\phi$ is a section of $Q_{1}(V)$ over a neighborhood of $a \in X$; then the diagram

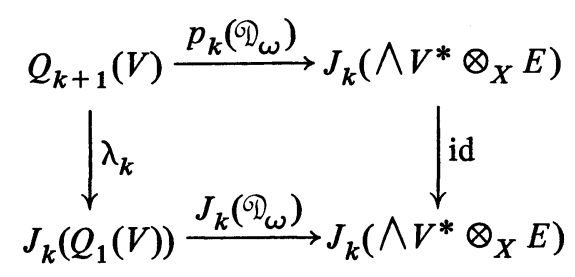

is commutative.

Let $Q_{k}(V ; \omega)$ be the sub-groupoid of $Q_{k}(V)$ consisting of the $k$-jets $\phi \in$ $Q_{k}(V)$ satisfying $p_{k-1}\left(D_{\omega}\right) \phi=0$. An element $\phi$ of $Q_{1}(V)$ belongs to $Q_{1}(V ; \omega)$ if and only if $\phi\left(\nu^{*-1} \omega\right)=\nu^{*-1} \omega$. By [9, Proposition 2.1], if $J_{k}(V ; \omega)$ is a vector 
bundle, then $Q_{k}(V ; \omega)$ is a fibered submanifold of $Q_{k}(V)$ and a differentiable groupoid which is a finite form of the Lie equation $J_{k}(V ; \omega)$, whose solutions are the local immersions $f: X \rightarrow X$ over $Y$ satisfying $f^{*} \omega=\omega$. Moreover from the commutativity of (22.24) and [9, Proposition 4.4], we deduce that if $J_{1}(V ; \omega)$ is a vector bundle, then

$$
\begin{aligned}
\lambda_{k} Q_{k+1}(V ; \omega) & =\lambda_{k} Q_{k+1}(V) \cap J_{k}\left(Q_{1}(V ; \omega)\right) \\
& =\lambda_{k} Q_{k+1} \cap J_{k}\left(Q_{1}(V ; \omega)\right),
\end{aligned}
$$

since $Q_{k+1}(V)=\left(Q_{1}(V)\right)_{+k}$; therefore if $J_{1}(V ; \omega)$ is a vector bundle, $Q_{k+1}(V ; \omega)$ is the $k$ th prolongation of $Q_{1}(V ; \omega)$. We write

$$
\tilde{\mathscr{Q}}_{k}(V ; \omega)=\tilde{\mathscr{Q}}_{k} \cap \mathscr{2}_{k}(V ; \omega) ;
$$

thus by Proposition 7.2, if $J_{1}(V ; \omega)$ is a vector bundle, we have

$$
\mathfrak{D}\left(\tilde{\mathcal{Q}}_{2}(V ; \omega)\right) \subset \mathcal{T}^{*} \otimes J_{1}(\mathfrak{V} ; \omega)
$$

The following lemma is needed for our study of $Q_{1}(V ; \omega)$ and provides us with a partial converse to Lemma 2.3:

Lemma 22.1. Let $\phi$ be a section of $\tilde{\mathcal{Q}}_{1}$, with $\pi_{0} \phi=f$, and $u$ a section of $\left(J_{0}(T)^{*} \otimes T\right)^{\wedge}$. Then the following are equivalent:

(i) $\overline{\mathfrak{D}} \phi=u$;

(ii) $\phi$ is the section $j_{1}(f)-f \circ u \circ \nu$ of $\tilde{\mathscr{Q}}_{1}$.

Proof. By Lemma 2.3 (ii), $j_{1}(f)-f \circ u \circ \nu$ is a section of $\tilde{\mathscr{Q}}_{1}$. According to Lemma 2.3 (iii), it suffices to prove the following: if $\phi, \phi_{1} \in \tilde{\mathcal{L}}_{1}$ satisfy $\pi_{0} \phi=\pi_{0} \phi_{1}$ and $\overline{\mathfrak{D}} \phi=\overline{\mathfrak{D}} \phi_{1}$, then $\phi=\phi_{1}$. In fact, by (2.41)

$$
\overline{\mathfrak{D}}\left(\phi \cdot \phi_{1}^{-1}\right)=\phi_{1}(\overline{\mathscr{D}} \phi)+\overline{\mathscr{D}} \phi_{1}^{-1}=\phi_{1}\left(\overline{\mathscr{D}} \phi_{1}\right)+\overline{\mathscr{D}} \phi_{1}^{-1}=\overline{\mathfrak{D}}\left(\phi_{1} \cdot \phi_{1}^{-1}\right)=0 \text {. }
$$

Therefore $\phi \cdot \phi_{1}^{-1}=j_{1}\left(\pi_{0}\left(\phi \cdot \phi_{1}^{-1}\right)\right)=j_{1}\left(I_{1}\right)$ and so $\phi=\phi_{1}$.

Recall that if $u \in\left(T^{*} \otimes J_{k}(T)\right)^{\wedge}$, then according to Lemma 2.2 we obtain an element $\bar{u} \in\left(J_{0}(T)^{*} \otimes \tilde{J}_{k}(T)\right)^{\wedge}$ such that

$$
\nu^{-1}-\bar{u}=(\nu+u)^{-1}: J_{k}(T) \rightarrow \tilde{J}_{k}(T) .
$$

If $k=0$, for $\alpha \in \wedge V^{*} \otimes_{X} E, \xi \in \wedge V$, we have

$$
\langle\xi, \alpha\rangle=\left\langle\left(\mathrm{id}+\nu^{-1} \circ u\right)(\mathrm{id}-\bar{u} \circ \nu) \xi, \alpha\right\rangle=\left\langle(\mathrm{id}-\bar{u} \circ \nu) \xi, \alpha^{u}\right\rangle .
$$

Proposition 22.5. Let $\omega, \alpha$ be sections of $\wedge V^{*} \otimes_{X} E$ and $u$ a section of $\left(T^{*} \otimes J_{0}(V)\right)^{\wedge}$ over $X$. Let $f$ be a local immersion $X \rightarrow X$ over $Y$. Then:

(i) $\phi=j_{1}(f)-f \circ \bar{u} \circ \nu$ is a section of $\tilde{\mathscr{Q}}_{1}(V)$ satisfying $\overline{\mathfrak{D}} \phi=\bar{u}$ and $\mathfrak{D} \phi=u$;

(ii) we have

$$
\left\langle\xi, \phi\left(\nu^{*-1} \omega\right)\right\rangle=\left\langle\left(\nu^{-1}-\bar{u}\right) \xi, f^{*} \omega\right\rangle,
$$

for $\xi \in \wedge J_{0}(V)$, and $\phi\left(\nu^{*-1} \omega\right)=\nu^{*-1} \alpha$ if and only if $f^{*} \omega=\alpha^{u}$;

(iii) $\phi$ is a section of $\tilde{\mathcal{Q}}_{1}(V ; \omega)$ if and only if $f^{*} \omega=\omega^{u}$. 
Proof. (i) By Lemmas 22.1 and 2.2 (iii), we need only show that $\phi$ is a section of $Q_{1}(V)$. By Lemma 2.3 (i), we have $\phi(\xi)=\nu\left(f\left(\nu^{-1}-\bar{u}\right) \xi\right)$, for $\xi \in J_{0}(T)$, and so $\rho \phi(\xi)=\rho \xi$. The desired result is a consequence of Proposition 6.1 (ii).

(ii) For $\xi \in \wedge J_{0}(V)^{*}$, we have

$$
\begin{aligned}
\left\langle\xi, \phi\left(\nu^{*-1} \omega\right)\right\rangle & =\left\langle\phi(\xi), \nu^{*-1} \omega\right\rangle=\left\langle\nu\left(f\left(\nu^{-1}-\bar{u}\right) \xi\right), \nu^{*-1} \omega\right\rangle \\
& =\left\langle\left(\nu^{-1}-\bar{u}\right) \xi, f^{*} \omega\right\rangle,
\end{aligned}
$$

and according to (22.26)

$$
\left\langle\xi, \nu^{*-1} \alpha\right\rangle=\left\langle\left(\nu^{-1}-\bar{u}\right) \xi, \alpha^{u}\right\rangle .
$$

Since $\nu^{-1}-\bar{u}: J_{0}(V) \rightarrow V$ is invertible, we deduce that $\phi\left(\nu^{*-1} \omega\right)=\alpha$ if and only if $f^{*} \omega=\alpha^{u}$.

(iii) is an immediate consequence of (i) and (ii), with $\alpha=\omega$.

From Lemma 22.1 and Proposition 22.5 (iii), we deduce that

$$
f^{*} \omega=\omega^{\mathscr{\phi}},
$$

for $\phi \in \tilde{\mathscr{Q}}_{1}(V ; \omega)$ with $f=\pi_{0} \phi$.

A section $u$ of $\left(T^{*} \otimes J_{1}(V ; \omega)\right)^{\wedge}$ determines a section $\omega^{u_{0}}$ of $\wedge V^{*} \otimes_{X} E$, where $u_{0}=\pi_{0} u$. In the next proposition, we investigate the relationship between the integrability condition $\mathfrak{D}_{1} u=0$ on $u$, an integrability condition (22.28) on $\omega^{u_{0}}$ and the existence of local diffeomorphisms $f: X \rightarrow X$ over $Y$ satisfying $f^{*} \omega=\omega^{u_{0}}$.

Proposition 22.6. Let $\omega$ be a section of $\wedge V^{*} \otimes_{X} E$ and $u_{0}$ a section of $\left(T^{*} \otimes J_{0}(V)\right)^{\wedge}$ over $X$. Let $f$ be a local diffeomorphism $X \rightarrow X$ over $Y$ defined on $a$ neighborhood of $a \in X$. Then of the following assertions, (i) and (ii) are equivalent while (iii) implies (iv):

(i) $f^{*} \omega=\omega^{u_{0}}$;

(ii) there is a section $\phi$ of $\tilde{\mathcal{Q}}_{1}(V ; \omega)$ over a neighborhood of a satisfying $\pi_{0} \phi=f$ and $\mathfrak{D} \phi=u_{0}$;

(iii) there is a section $u$ of $\left(T^{*} \otimes J_{1}(V ; \omega)\right)^{\wedge}$ such that $\pi_{0} u=u_{0}$ and $\mathfrak{D}_{1} u=0$ on a neighborhood $U$ of $a$;

(iv) we have

$$
d_{X / Y} \omega^{u_{0}}=\left(d_{X / Y} \omega\right)^{u_{0}}
$$

on a neighborhood $U$ of $a$.

Moreover, if $J_{1}(V ; \omega)$ is a vector bundle and the finite form $Q_{1}(V ; \omega)$ of $J_{1}(V ; \omega)$ is formally integrable, assertion (i) or (ii) implies (iii). 
Proof. The equivalence of (i) and (ii) is a consequence of Lemma 22.1 and Proposition 22.5. Next assume that (iii) holds. If we write $\tilde{J}_{1}(V ; \omega)=$ $\nu^{-1} J_{1}(V ; \omega)$, then $\hat{u}=\left(\nu^{*-1} \otimes \nu^{-1}\right)\left(u_{\mid V}\right)$ is a section of $J_{0}(V)^{*} \otimes \tilde{J}_{1}(V ; \omega)$, and so

$$
\mathcal{L}_{v}(\hat{u})\left(\nu^{*-1} \omega\right)=0
$$

where $v=u_{0 \mid V}$. Since $\mathfrak{D}_{1, X / Y}\left(u_{\mid V}\right)=0$, we apply Proposition 22.3 to $\omega$ and $u_{\mid V}$ to obtain (iv). Finally, assume that $J_{1}(V ; \omega)$ is a vector bundle and that $Q_{1}(V ; \omega)$ is formally integrable, and that (ii) holds. There is a section $\psi$ of $\tilde{\mathcal{Q}}_{2}(V ; \omega)$ over a neighborhood of $a$ such that $\pi_{1} \psi=\phi$; then by $(22.25), u=\mathfrak{D} \psi$ is a section of $\left(T^{*} \otimes J_{1}(V ; \omega)\right)^{\wedge}$ satisfying $\pi_{0} u=u_{0}$ and $\mathfrak{D}_{1} u=0$.

The proof of part (i) of the following proposition resembles the argument used to prove Proposition 7.6 (see also the end of the proof of Theorem 8.1).

Proposition 22.7. Let $R_{k} \subset J_{k}(T)$ be a formally integrable Lie equation. Let $P_{k} \subset Q_{k}$ be a finite form of $R_{k}$ whose lth prolongation we denote by $P_{k+l}$. Assume that $\pi_{k}: P_{k+1} \rightarrow P_{k}$ is surjective.

(i) If $u \in \bar{Z}^{1}\left(R_{k}\right)_{a}$, with $a \in X$, and $\hat{u}$ is the image of $u$ in $\hat{Z}^{1}\left(R_{k}\right)_{a}$ under the mapping (7.13) and $\phi \in \tilde{\mathscr{P}}_{k, a}$, then the following assertions are equivalent:

(a) $\overline{\mathfrak{D}} \phi=\pi_{k-1} u$;

(b) $\hat{\mathfrak{D}} \phi=\hat{u}$;

(c) there is an element $\phi_{1} \in \tilde{\mathscr{\rho}}_{k+1}$ satisfying

$$
\pi_{k} \phi_{1}=\phi, \quad \overline{\mathfrak{D}} \phi_{1}=u \text {. }
$$

(ii) Suppose that $P_{k}$ is a formally integrable and integrable finite form of $R_{k}$. If $u \in \bar{Z}^{1}\left(R_{k}\right)_{a}$ and $\hat{u}$ is the image of $u$ in $\hat{Z}^{1}\left(R_{k}\right)_{a}$, then the following assertions are equivalent:

(a) the cohomology class of $u$ in $H^{1}\left(P_{k}\right)_{k, a}$ vanishes;

(b) the cohomology class of $\hat{u}$ in $\hat{H}^{1}\left(P_{k}\right)_{k, a}$ vanishes;

(c) there exists $\phi \in \tilde{\mathscr{P}}_{k, a}$ satisfying $\overline{\mathfrak{D}}_{\phi}=\pi_{k-1} u$.

(iii) Suppose that $P_{k}$ is a formally integrable and integrable finite form of $R_{k}$. If for all $u \in Z^{1}\left(R_{k}\right)_{a}$, with $a \in X$, we can solve the equation $\overline{\mathfrak{D}} \phi=\pi_{k-1} u$, for $\phi \in \tilde{\mathscr{P}}_{k, a}$, then $\bar{H}^{1}\left(P_{k}\right)_{m, a}=0$, for all $m \geqslant k, a \in X$.

Proof. (i) Clearly (c) $\Rightarrow$ (b) $\Rightarrow$ (a). We now verify that (a) $\Rightarrow$ (c). Suppose $u \in \bar{Z}^{1}\left(R_{k}\right)_{a}$ and $\phi \in \tilde{\mathscr{P}}_{k, a}$ satisfy (a) with $\left(\pi_{0} \phi\right)(a)=b$. Choose $\psi_{1} \in \tilde{\mathscr{P}}_{k+1, a}$ with $\pi_{k} \psi_{1}=\phi$. Since $\left(\pi_{k-1} u\right)^{\phi^{-1}}=0$, we have

$$
u^{\psi_{1}^{-1}} \in\left(J_{0}(\tau) * \otimes g_{k}\right)_{b}
$$

and (see §1)

$$
\bar{\delta} u^{\psi_{1}^{-1}}=-\bar{D} u^{\psi_{1}^{-1}}=-\frac{1}{2} \pi_{k}\left[u^{\psi_{1}^{-1}}, u^{\psi_{1}^{-1}}\right]=0
$$


Since $g_{k}$ is 1-acyclic, there exists $v \in g_{k+1}$ such that $\bar{\delta} v=u^{\psi_{1}^{-1}}$. According to [9, Proposition 7.1], $\pi_{k}: P_{k+1} \rightarrow P_{k}$ is an affine sub-bundle of $\pi_{k}: Q_{k+1 \mid P_{k}} \rightarrow P_{k}$, and so the mapping (7.4), with $l=0$, namely

$$
\text { ว: } Q_{k+1}^{k} \cap P_{k+1} \rightarrow g_{k+1},
$$

is an isomorphism of Lie groups over $X$ and thus $\psi_{2}=\partial^{-1} v$ belongs to $2_{k+1}^{k} \cap \mathscr{P}_{k+1}$. By (2.38),

$$
\overline{\mathfrak{D}} \psi_{2}=-\bar{\delta} v=-u^{\psi_{1}^{-1}}
$$

and $\psi_{1}^{-1} \cdot \psi_{2} \in \tilde{\mathscr{P}}_{k+1, b}$, with $\pi_{0}\left(\psi_{1}^{-1} \cdot \psi_{2}\right)(b)=a$; then

$$
u^{\psi_{1}^{-1} \cdot \psi_{2}}=u^{\psi_{1}^{-1}}+\overline{\mathfrak{D}} \psi_{2}=0
$$

Hence $\phi_{1}=\psi_{2}^{-1} \cdot \psi_{1} \in \tilde{\mathscr{P}}_{k+1, a}$ satisfies $\pi_{k} \phi_{1}=\phi$ and $\overline{\mathfrak{D}} \phi_{1}=u$.

(ii) Clearly (a) $\Rightarrow$ (b) $\Rightarrow$ (c). We now verify that (c) $\Rightarrow$ (a). If $u \in \bar{Z}^{1}\left(R_{k}\right)_{a}$ and $\phi \in \tilde{\mathscr{P}}_{k, a}$ satisfy $\overline{\mathfrak{D}} \phi=\pi_{k-1} u$, then according to (i) there exists $\phi_{1} \in \tilde{\mathscr{P}}_{k+1, a}$, with $\pi_{k} \phi_{1}=\phi$, such that $\bar{D} \phi_{1}=u$. Since $P_{k}$ is integrable, there exists $f \in$ $\operatorname{Sol}\left(P_{k}\right)_{a}$ such that $j_{k+1}(f)(a)=\phi_{1}(a)$. Then $j_{k+1}\left(f^{-1}\right) \cdot \phi_{1}$ belongs to $\tilde{\mathscr{P}}_{k+1, a}^{\cdot}$, and by (2.41)

$$
\overline{\mathfrak{D}}\left(j_{k+1}\left(f^{-1}\right) \cdot \phi_{1}\right)=\overline{\mathfrak{D}} \phi_{1}=u .
$$

(iii) is a consequence of (ii) and Proposition 7.8.

From Propositions 22.5 and 22.7 (iii), we obtain directly:

Proposition 22.8. Let $\omega$ be a section of $\wedge V^{*} \otimes_{X} E$. Assume that $J_{1}(V ; \omega)$ is a formally integrable Lie equation and that $Q_{1}(V ; \omega)$ is a formally integrable and integrable finite form of $J_{1}(V ; \omega)$. If for all $u \in\left(\mathcal{T}^{*} \otimes J_{1}(\mathcal{V} ; \omega)\right)_{a}^{\wedge}$, with $a \in X$, satisfying $\mathfrak{D}_{1} u=0$, there is a local diffeomorphism $f: X \rightarrow X$ over $Y$ defined on a neighborhood of a satisfying $f^{*} \omega=\omega^{u_{0}}$, where $u_{0}=\pi_{0} u$, then

$$
\bar{H}^{1}\left(Q_{1}(V ; \omega)\right)_{m, a}=0, \quad \tilde{H}^{1}\left(J_{1}(V ; \omega)\right)_{a}=0,
$$

for all $m \geqslant 1, a \in X$.

The sub-bundle $Q_{1}(V)$ of $Q_{1}$ is a formally integrable and integrable finite form of the formally integrable Lie equation $J_{1}(V)$, and its $k$ th prolongation is $Q_{k+1}(V)$. The following proposition is a special case of Theorem 8.1 and also follows directly from Proposition 22.8 with $\omega=0$, taking $f$ to be the identity mapping of $X$.

Proposition 22.9. For all $m \geqslant 1, a \in X$, we have

$$
\bar{H}^{1}\left(Q_{1}(V)\right)_{m, a}=0, \quad \tilde{H}^{1}\left(J_{1}(V)\right)_{a}=0 .
$$

By the exactness of (1.3), we also have

$$
H^{j}\left(J_{1}(V)\right)=0, \text { for } j>0 .
$$


If $x \in X$, there are structures of analytic manifolds on a neighborhood $U$ of $x$ in $X$ and on $\rho(U)$ compatible with the structures of differentiable manifolds on $X$ and $Y$ such that $\rho_{\mid U}$ is analytic. Then $J_{1}(T ; \rho)$ is a formally transitive and formally integrable analytic Lie equation; since $J_{1}(V) \subset J_{1}(T ; \rho)$ is a formally integrable Lie equation satisfying (6.3), for $x \in X$ and $j>0$ we have

$$
\begin{aligned}
& H^{j}\left(J_{\infty}(T ; \rho)_{x}, J_{\infty}(V)_{x}\right)=H^{j}\left(J_{1}(V)\right)_{x}=0, \\
& \tilde{H}^{1}\left(J_{\infty}(T ; \rho)_{x}, J_{\infty}(V)_{x}\right)=\tilde{H}^{1}\left(J_{1}(V)\right)_{x}=0,
\end{aligned}
$$

by Proposition 22.9 .

Let $Z$ be a manifold and assume that $\rho: X \rightarrow Y$ is the fibered manifold $\mathrm{pr}_{1}$ : $Y \times Z \rightarrow Y$. If $x=(y, z) \in X$, from the properties of the isomorphism (21.4) of transitive Lie algebras given in $\S 21$ and (22.30), we deduce:

Proposition 22.10. If $Z$ is a manifold, then for $z \in Z$ and $y \in Y$, we have

$$
H^{j}\left(\left(J_{\infty}\left(T_{Z} ; Z\right)_{z} \hat{\otimes} F_{y}\right) \oplus J_{\infty}\left(T_{Y} ; Y\right)_{y}, J_{\infty}\left(T_{Z} ; Z\right)_{z} \hat{\otimes} F_{y}\right)=0
$$

for $j>0$, and

$$
\tilde{H}^{1}\left(\left(J_{\infty}\left(T_{Z} ; Z\right)_{z} \hat{\otimes} F_{y}\right) \oplus J_{\infty}\left(T_{Y} ; Y\right)_{y}, J_{\infty}\left(T_{Z} ; Z\right)_{z} \hat{\otimes} F_{y}\right)=0 .
$$

Finally, we assume again that $\rho: X \rightarrow Y$ is an arbitrary surjective submersion, and further that $E$ is a trivial vector bundle over $Y$. If $\xi$ is a $\rho$-projectable vector field on $X$, formula (22.17) defines a section $\mathcal{L}(\xi) \omega$ of $\wedge V^{*} \otimes_{X} E$, while formula (22.18) determines an element $\mathcal{L}(\tilde{\xi}) \alpha$ of $\wedge J_{0}(V)^{*} \otimes_{X} E$, for all $\tilde{\xi} \in \tilde{J}_{1}(T ; \rho), \alpha \in \wedge J_{0}(\mathscr{V})^{*} \otimes \mathcal{E}_{X}$. The differential operator

$$
D_{\omega}: \mathcal{T}_{\rho} \rightarrow \wedge \mathcal{V}^{*} \otimes \mathcal{E}_{X},
$$

sending $\xi$ into $\mathcal{L}(\xi) \omega$, extends the differential operator considered above; the morphism of vector bundles

$$
p_{k-1}\left(D_{\omega}\right): J_{k}(T ; \rho) \rightarrow J_{k-1}\left(\wedge V^{*} \otimes_{X} E\right)
$$

determined by this operator $D_{\omega}$, sending $j_{k}(\xi)(x)$, with $\xi \in \mathcal{T}_{\rho, x}$, into $j_{k-1}\left(D_{\omega} \xi\right)(x)$, is an extension of the morphism $p_{k-1}\left(D_{\omega}\right)$ considered above. The kernel $J_{k}(T ; \omega)$ of $(22.31)$ is a sub-bundle of $J_{k}(T ; \rho)$ with possibly varying fiber and satisfies

$$
J_{k}(V ; \omega)=J_{k}(V) \cap J_{k}(T ; \omega) .
$$

An element $\xi$ of $J_{1}(T ; \rho)$ belongs to $J_{1}(T ; \omega)$ if and only if $\mathcal{L}(\tilde{\xi}) \nu^{*-1} \omega=0$, where $\tilde{\xi}=\nu^{-1} \xi$. It follows that if $J_{1}(T ; \omega)$ is a vector bundle, it is a Lie equation whose $k$ th prolongation is $J_{k+1}(T ; \omega)$. Moreover, we have the exact sequence

$$
0 \rightarrow J_{k}(V ; \omega)_{x} \rightarrow J_{k}(T ; \omega)_{x} \stackrel{\rho}{\rightarrow} J_{k}\left(T_{Y} ; Y\right)_{\rho(x)}
$$


for $x \in X$, and

$$
\begin{aligned}
& {\left[J_{k+1}(T ; \omega), J_{k+1}(T ; \omega)\right] \subset J_{k}(T ; \omega),} \\
& {\left[J_{k+1}(T ; \omega), J_{k+1}(V ; \omega)\right] \subset J_{k}(V ; \omega),}
\end{aligned}
$$

for $k \geqslant 1$. For $x \in X$, we write

$$
\begin{aligned}
& J_{\infty}(T ; \omega)_{x}=\lim _{\leftarrow} J_{k}(T ; \omega)_{x}, \\
& J_{\infty}(V ; \omega)_{x}=\underset{\leftarrow}{\lim } J_{k}(V ; \omega)_{x} ;
\end{aligned}
$$

by $(22.32), J_{\infty}(V ; \omega)_{x}$ is a closed ideal of the linearly compact Lie algebra $J_{\infty}(T ; \omega)_{x}$.

Let $Z$ be a manifold and assume that $\rho: X \rightarrow Y$ is the fibered manifold $\operatorname{pr}_{1}$ : $Y \times Z \rightarrow Y$. Denote by $V^{\prime}$ the integrable sub-bundle of $T$ consisting of vectors tangent to the fibers of $\mathrm{pr}_{2}: Y \times Z \rightarrow Z$. We have the decomposition

$$
T=V \oplus V^{\prime} \text {, }
$$

and $\rho$ induces an isomorphism

$$
\rho: V^{\prime} \rightarrow \rho^{-1} T_{Y} .
$$

If $\zeta \in \Gamma\left(Y, T_{Y}\right)$, then $\xi=\rho^{-1} \zeta$ is the unique $\rho$-projectable section of $V^{\prime}$ satisfying $\rho \xi=\zeta$. Clearly $J_{1}\left(V^{\prime} ; \rho\right)$ is a formally integrable Lie equation whose $k$ th prolongation is $J_{k+1}\left(V^{\prime} ; \rho\right)$ and $\rho$ induces an isomorphism

$$
\rho: J_{k}\left(V^{\prime} ; \rho\right) \rightarrow \rho^{-1} J_{k}\left(T_{Y} ; Y\right),
$$

whose inverse we denote by

$$
\sigma: \rho^{-1} J_{k}\left(T_{Y} ; Y\right) \rightarrow J_{k}\left(V^{\prime} ; \rho\right) .
$$

For $x \in X$, with $y=\rho(x)$, and $\zeta \in \Gamma\left(Y, T_{Y}\right)$, the mapping (22.33) sends $\left(x, j_{k}(\zeta)(y)\right)$ into

$$
\sigma_{x}\left(j_{k}(\zeta)(y)\right)=j_{k}\left(\rho^{-1} \zeta\right)(x)
$$

and we have

$$
\left[\sigma_{x} \xi, \sigma_{x} \eta\right]
$$

for $\xi, \eta \in J_{k}\left(T_{Y} ; Y\right)_{y}$. The mapping

$$
\sigma_{x}: J_{\infty}\left(T_{Y} ; Y\right)_{y} \rightarrow J_{\infty}(T ; \rho)_{x}
$$

determined by (22.33) with $k=\infty$ is equal to the mapping (21.2) and its image is $J_{\infty}\left(V^{\prime} ; \rho\right)_{x}$. Moreover, we have the decomposition

$$
J_{k}(T ; \rho)=J_{k}(V) \oplus J_{k}\left(V^{\prime} ; \rho\right) ;
$$

for $k=\infty$, this gives us a decomposition of $J_{\infty}(T ; \rho)_{x}$ as the semi-direct product of its closed subalgebra $J_{\infty}\left(V^{\prime} ; \rho\right)_{x}$ and its closed ideal $J_{\infty}(V)_{x}$. 
Let $\omega_{Z}$ be a form on $Z$ with values in the vector space $E$. We assume now that the vector bundle $E$ is the trivial vector bundle over $Y$ whose fiber is the vector space $E$ and that $\omega$ is the section $\tilde{\omega}_{Z}$ of $\wedge V^{*} \otimes_{X} E$ determined by the form $\omega_{Z}$. The $\rho$-projectable vector field $\rho^{-1} \xi$ on $X$ determined by a vector field $\xi$ on $Y$ clearly satisfies the relation $\mathcal{L}\left(\rho^{-1} \xi\right) \omega=0$. Hence we have the decomposition

$$
J_{k}(T ; \omega)=J_{k}(V ; \omega) \oplus J_{k}\left(V^{\prime} ; \rho\right) .
$$

Moreover for $x=(y, z) \in Y \times Z$, the image $J_{\infty}\left(V^{\prime} ; \rho\right)_{x}$ of the morphism of Lie algebras $(21.2)$ is contained in $J_{\infty}(T ; \omega)_{x}$ and $J_{\infty}(V ; \omega)_{x}$ is a $J_{\infty}\left(T_{Y} ; Y\right)_{y^{-}}$ submodule of $J_{\infty}(V)_{x}$, and $J_{\infty}(T ; \omega)_{x}$ is the semi-direct product of its closed subalgebra $J_{\infty}\left(V^{\prime} ; \rho\right)_{x}$ and its closed ideal $J_{\infty}(V ; \omega)_{x}$. Therefore the sequence

$$
0 \rightarrow J_{k}(V ; \omega)_{x} \rightarrow J_{k}(T ; \omega)_{x} \stackrel{\rho}{\rightarrow} J_{k}\left(T_{Y} ; Y\right)_{\rho(x)} \rightarrow 0
$$

is exact for $1 \leqslant k \leqslant \infty$. It is easily seen that the image of the semi-direct product

$$
\left(J_{\infty}\left(T_{Z} ; \omega_{Z}\right)_{z} \hat{\otimes} F_{y}\right) \oplus J_{\infty}\left(T_{Y} ; Y\right)_{y}
$$

under the isomorphism (21.4) is equal to $J_{\infty}(T ; \omega)_{x}$ and that the restriction

$$
\lambda: J_{\infty}\left(T_{Z} ; \omega_{Z}\right)_{z} \hat{\otimes} F_{y} \rightarrow J_{\infty}(V ; \omega)_{x}
$$

of the mapping (21.3) is an isomorphism of $J_{\infty}\left(T_{Y} ; Y\right)_{y}$-modules.

\section{Some simple Lie algebras with parameters}

Let $q$ be the rank of $V$ and assume throughout this section that $q \geqslant 1$. Let $E$ be a vector bundle over $Y$ and $r \geqslant 1$. A section $\omega$ of $\wedge^{r} V^{*} \otimes_{X} E$ over $X$ is said to be non-singular if the mapping

$$
\omega: V \rightarrow \wedge^{r-1} V^{*} \otimes_{X} E
$$

sending $\xi$ into $\xi \pi \omega$, is an isomorphism of vector bundles over $X$.

Proposition 23.1. Let $\omega$ be a non-singular section of $\wedge^{r} V^{*} \otimes_{X} E$ over $X$.

(i) If $u$ is a section of $T^{*} \otimes J_{0}(V)$ over $X$, then $u$ is a section of $\left(T^{*} \otimes J_{0}(V)\right)^{\wedge}$ if and only if $\omega^{u}$ is a non-singular section of $\wedge^{r} V^{*} \otimes_{X} E$ over $X$.

(ii) If $f$ is a local mapping $X \rightarrow X$ over $Y$ defined on an open set $U$, then $f$ is an immersion of $U$ if and only if $f^{*} \omega$ is a non-singular section of $\wedge^{r} V^{*} \otimes_{X} E$ over $U$. 
Proof. (i) If $u \in T^{*} \otimes J_{0}(V)$ and $v=\left(\mathrm{id}+\nu^{-1} \circ u\right)_{\mid V}$, the diagram

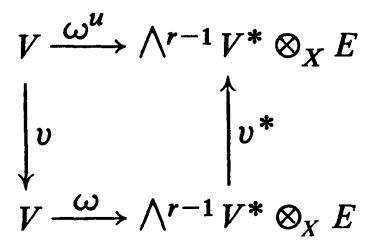

commutes; the mapping $\omega^{u}$ is an isomorphism if and only if the mapping $v$ is an isomorphism, or equivalently if $u \in\left(T^{*} \otimes J_{0}(V)\right)^{\wedge}$.

(ii) For $x \in U$, the diagram

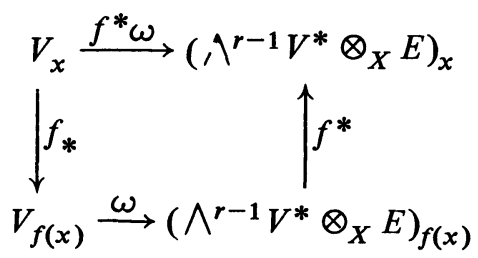

is commutative; thus the mapping $f^{*} \omega$ of the diagram is an isomorphism if and only if $f_{*}: V_{x} \rightarrow V_{f(x)}$ is an isomorphism, or equivalently if and only if $f$ is an immersion on a neighborhood of $x$.

In this section and the next, we shall examine three types of non-singular sections of the vector bundles $\wedge^{r} V^{*} \otimes_{X} E$ and the corresponding Lie equations. For the remainder of this section, we assume that $E=1$ and that $r=q$ or $r=2$.

A section of $\wedge{ }^{q} V^{*}$ is non-singular if and only if it does not vanish at any point of $X$. A non-singular section $\omega$ of $\wedge^{q} V^{*}$ is called a volume form along the fibers of $\rho$; the restriction $\omega_{X_{y}}$ of $\omega$ to a fiber $X_{y}=\rho^{-1}(y)$, with $y \in Y$, is a volume form on $X_{y}$. If $\omega$ is a non-singular section of $\wedge^{2} V^{*}$, then the rank of $V$ is even and equal to $q=2 p$, with $p \geqslant 1$; if $q$ is even and equal to $2 p$, a section $\omega$ of $\wedge^{2} V^{*}$ is non-singular if and only if the section $\omega^{p}$ of $\wedge^{q} V^{*}$ does not vanish at any point of $X$. A non-singular section $\omega$ of $\wedge^{2} V^{*}$ is called a symplectic form along the fibers of $\rho$ if $d_{X / Y} \omega=0$; the restriction $\omega_{X_{y}}$ of such a symplectic form $\omega$ to a fiber $X_{y}$, with $y \in Y$, is a symplectic form on $X_{y}$. If $X$ is the product $Y \times Z$ of $Y$ with a manifold $Z$ and $\rho: X \rightarrow Y$ is the projection onto the first factor, and if $\omega_{Z}$ is a volume or symplectic form on $Z$, the section $\tilde{\omega}_{Z}$ of $\wedge^{r} V^{*}$ determined by $\omega_{Z}$ is a volume or symplectic form along the fibers of $\rho$. 
Proposition 23.2. Let $\omega, \omega^{\prime}$ be two non-singular sections of $\wedge^{r} V^{*}$. If $r=2$ or $r=q$, then for all $a \in X$, there is a section $u_{0}$ of $\left(T^{*} \otimes J_{0}(V)\right)^{\wedge}$ such that $\omega^{\prime}=\omega^{u_{0}}$ on a neighborhood of $a$.

Proof. There are frames $\left\{\alpha_{1}, \cdots, \alpha_{q}\right\},\left\{\alpha_{1}^{\prime}, \cdots, \alpha_{q}^{\prime}\right\}$ for $V^{*}$ over a neighborhood $U$ of $a \in X$ such that

$$
\omega=\alpha_{1} \wedge \cdots \wedge \alpha_{q}, \quad \omega^{\prime}=\alpha_{1}^{\prime} \wedge \cdots \wedge \alpha_{q}^{\prime},
$$

when $r=q$, or such that

$$
\omega=\sum_{j=1}^{p} \alpha_{2 j-1} \wedge \alpha_{2 j}, \quad \omega^{\prime}=\sum_{j=1}^{p} \alpha_{2 j-1}^{\prime} \wedge \alpha_{2 j}^{\prime},
$$

when $r=2$, according to Cartan's lemma. Let $v$ be the section of $V^{*} \otimes V$ over $U$ satisfying $v^{*}\left(\alpha_{j}\right)=\alpha_{j}^{\prime}$, for $1 \leqslant j \leqslant q$, and let $u_{0}$ be a section of $T^{*} \otimes J_{0}(V)$ over $U$ satisfying (id $\left.+\nu^{-1} \circ u_{0}\right)_{\mid V}=v$. Then $\omega^{\prime}=\omega^{u_{0}}$, and $u_{0}$ is a section of $\left(T^{*} \otimes J_{0}(V)\right)^{\wedge}$ by Proposition 23.1 (i).

Let $\omega$ be a non-singular section of $\wedge^{r} V^{*}$. Assume that either $r=q$, or that $r=2$ and $\omega$ is symplectic. Since $d_{X / Y} \omega=0$, by (22.18) the hypotheses of Proposition 22.4 are satisfied with $\sigma$ equal to the mapping (23.1) and $\tau$ to the identity mapping of $\wedge^{r} V^{*}$. Therefore $J_{1}(V ; \omega)$ is a formally integrable Lie equation whose $k$ th prolongation is $J_{k+1}(V ; \omega)$, and $Q_{1}(V ; \omega)$ is a finite form of $J_{1}(V ; \omega)$ whose $k$ th prolongation is $Q_{k+1}(V ; \omega)$; moreover $\pi_{0}\left(J_{1}(V ; \omega)\right)=$ $J_{0}(V)$ and (22.21) holds.

Let $(w, y)$ be a local coordinate for $X$ on an open neighborhood $U$ of $a \in X$ compatible with $\rho$, that is, $w=\left(w^{1}, \cdots, w^{q}\right)$ is a coordinate along the fibers of $\rho$ and $y=\left(y^{1}, \cdots, y^{m}\right)$ is a local coordinate for $Y$ on $\rho(U)$. First, suppose that $\omega$ is a volume form along the fibers of $\rho$; then $\omega$ is the restriction to $\wedge^{q} V$ of the $q$-form

$$
h d w^{1} \wedge \cdots \wedge d w^{q},
$$

where $h$ is a real-valued function on $U$. We solve the equation $\partial g / \partial w^{1}=h$ for a real-valued function $g$ on a neighborhood of $a$. If $z^{1}=g, z^{j}=w^{j}$ for $j \geqslant 2$, then $\omega$ is the restriction to $\wedge{ }^{q} V$ of the $q$-form

$$
d z^{1} \wedge d z^{2} \wedge \cdots \wedge d z^{q},
$$

and $\left(z^{1}, \cdots, z^{q}, y^{1}, \cdots, y^{m}\right)$ is a local coordinate on a neighborhood $U^{\prime}$ of $a \in X$ compatible with $\rho$. A vertical vector field $\xi=\sum_{j=1}^{q} \xi^{j} \partial / \partial z^{j}$ on $U^{\prime}$ is a solution of $J_{1}(V ; \omega)$ if and only if

$$
\sum_{j=1}^{q} \frac{\partial \xi^{j}}{\partial z^{j}}=0
$$


Next, if $\omega$ is a symplectic form along the fibers of $\rho$, according to Darboux's theorem with parameters, there are functions $z^{1}, \cdots, z^{q}$ on a neighborhood of $a$ such that $\omega$ is the restriction to $\wedge^{2} V$ of the 2-form

$$
\sum_{j=1}^{p} d z^{2 j-1} \wedge d z^{2 j}
$$

Since $\omega$ is non-singular, $\left(z^{1}, \cdots, z^{q}, y^{1}, \cdots, y^{m}\right)$ is a local coordinate on a neighborhood $U^{\prime}$ of $a$ compatible with $\rho$. In either case, the vertical vector field

$$
\xi_{0}=\sum_{j=1}^{q} z^{j} \frac{\partial}{\partial z^{j}}
$$

on $U^{\prime}$ satisfies $\mathcal{L}\left(\xi_{0}\right)=r \omega$ and $\mathcal{L}\left(\rho^{*} f \cdot \xi_{0}\right)=r \rho^{*} f \cdot \omega$, if $f$ is a real-valued function on $\rho\left(U^{\prime}\right)$. Clearly we may assume that $z^{j}(a)=0$ for $1 \leqslant j \leqslant q$; then we have $\xi_{0}(a)=0$.

Proposition 23.3. (i) Let $\omega, \omega^{\prime}$ be two volume or two symplectic forms along the fibers of $\rho$. If $a, b \in X$, with $\rho(a)=\rho(b)$, there is a local diffeomorphism $f: X \rightarrow X$ over $Y$ defined on $a$ neighborhood of $a$ such that $f(a)=b$ and $f^{*} \omega=\omega^{\prime}$

(ii) If $\omega$ is a volume or a symplectic form along the fibers of $\rho$, then $Q_{1}(V ; \omega)$ is a formally integrable and integrable finite form of the formally integrable Lie equation $J_{1}(V ; \omega)$, whose kth prolongation is $Q_{k+1}(V ; \omega)$.

Proof. (i) There are local coordinates $(z, y)$ on a neighborhood $U$ of $a$ and $\left(z^{\prime}, y\right)$ on a neighborhood $U^{\prime}$ of $b$ compatible with $\rho$, where $y=\left(y^{1}, \cdots, y^{m}\right)$ is a local coordinate for $Y$ on a neighborhood of $\rho(a)$. From the above discussion, if $\omega$ and $\omega^{\prime}$ are volume forms, we may assume that $\omega$ is the restriction to $\wedge{ }^{q} V$ of the $q$-form $d z^{1} \wedge \cdots \wedge d z^{q}$ on $U$ and that $\omega^{\prime}$ is the restriction to $\wedge{ }^{q} V$ of the $q$-form $d z^{\prime 1} \wedge \cdots \wedge d z^{\prime q}$ on $U^{\prime}$; similarly, if $\omega$ and $\omega^{\prime}$ are symplectic forms, we may suppose that $\omega$ is the restriction to $\wedge^{2} V$ of the 2-form $\sum_{j=1}^{p} d z^{2 j-1} \wedge d z^{2 j}$ on $U$ and that $\omega^{\prime}$ is the restriction to $\wedge^{2} V$ of the 2-form $\sum_{j=1}^{p} d z^{2 j-1} \wedge d z^{\prime 2 j}$ on $U^{\prime}$. We may also suppose that $z(a)=z^{\prime}(b)$. The mapping $f$ defined on a neighborhood of $a$ sending the point of $U$ with coordinate $(z, y)$ into the point of $U^{\prime}$ whose $\left(z^{\prime}, y\right)$-coordinate is equal to $(z, y)$ is a diffeomorphism over $Y$ and satisfies $f(a)=b$ and $f^{*} \omega=\omega^{\prime}$, since $z^{\prime} \circ f=z$.

(ii) We begin by proving that for $\phi \in Q_{1}(V ; \omega)$, with source $\phi=a$, there is a local diffeomorphism $f: X \rightarrow X$ over $Y$ defined on a neighborhood of $a$ such that $j_{1}(f)(a)=\phi$ and $f^{*} \omega=\omega$. If we take $\omega^{\prime}=\omega$ in (i), we see that it suffices to verify this assertion when target $\phi$ is also equal to $a$. Assume that $(z, y)$ is a local coordinate compatible with $\rho$ on a neighborhood $U$ of $a$, with $z(a)=0$ and $y(\rho(a))=0$, satisfying the conditions with respect to $\omega$ described in the 
proof of (i). Let $\phi \in Q_{1}(V)$ with source $\phi=\operatorname{target} \phi=a$. If $\phi: J_{0}(T)_{a} \rightarrow J_{0}(T)_{a}$ sends $\nu\left(\partial / \partial z^{j}\right)$ into $\sum_{l=1}^{q} \alpha_{j}^{l} \nu\left(\partial / \partial z^{l}\right)$, for $1 \leqslant j \leqslant q$, and $\nu\left(\partial / \partial y^{i}\right)$ into $\nu\left(\partial / \partial y^{i}\right.$ $\left.+\sum_{l=1}^{m} \beta_{i}^{l} \partial / \partial z^{l}\right)$, for $1 \leqslant i \leqslant m$, then the mapping $f$ defined by

$$
f(z, y)=\left(f^{1}(z, y), \cdots, f^{q}(z, y), y^{1}, \cdots, y^{m}\right),
$$

with

$$
f^{j}(z, y)=\sum_{l=1}^{q} \alpha_{l}^{j} z^{l}+\sum_{i=1}^{m} \beta_{i}^{j} y^{i},
$$

is a local diffeomorphism of $X$ over $Y$ on a neighborhood of $a$ which satisfies $j_{1}(f)(a)=\phi$; moreover $f^{*} \omega=\omega$ if and only if $\phi\left(\nu^{*-1} \omega\right)=\nu^{*-1} \omega$, or if $\phi \in$ $Q_{1}(V ; \omega)$. Thus we have verified our assertion, which implies that $\pi_{1}: Q_{2}(V ; \omega)$ $\rightarrow Q_{1}(V ; \omega)$ is surjective. Since $g_{1}(V ; \omega)$ is 2-acyclic and $g_{k}(V ; \omega)$ is a vector bundle for $k \geqslant 1$ by Proposition 22.4, we apply [9, Theorem 8.1] to $Q_{1}(V ; \omega)$ and deduce that it is a formally integrable finite form of $J_{1}(V ; \omega)$. With respect to the analytic structure on $U$ determined by the coordinate $(z, y)$, the form $\omega$ is analytic and the open sub-bundle of $Q_{1}(V ; \omega)_{\mid U}$ consisting of all elements $\phi \in Q_{1}(V ; \omega)$ with source and target belonging to $U$ is an analytic and formally integrable differential equation on $U$, and therefore integrable. That $Q_{1}(V ; \omega)$ is integrable now follows from (i) with $\omega=\omega^{\prime}$.

The equivalence of assertions (i) and (ii) of the following proposition shows that the mapping $T^{*} \otimes J_{1}(V) \rightarrow \wedge^{r} V^{*}$, sending $u$ into $\omega^{u_{0}}$, with $u_{0}=\pi_{0} u$, induces a surjective mapping from $Z^{1}\left(J_{1}(V ; \omega)\right)_{a}$ to the set of germs at $a \in X$ of volume or symplectic forms along the fibers of $\rho$.

Proposition 23.4. Let $\omega$ be a volume (resp. symplectic) form along the fibers of $\rho$ and let $a \in X$. If $\omega^{\prime}$ is a section of $\wedge^{q} V^{*}$ (resp. $\left.\wedge^{2} V^{*}\right)$ over a neighborhood of $a$, the following assertions are equivalent:

(i) $\omega^{\prime}$ is a volume (resp. symplectic) form along the fibers of $\rho$ on a neighborhood of $a$;

(ii) there is a section $u$ of $\left(T^{*} \otimes J_{1}(V ; \omega)\right)^{\wedge}$ such that $\mathscr{D}_{1} u=0$ and $\omega^{\prime}=\omega^{u_{0}}$, with $u_{0}=\pi_{0} u$, on a neighborhood of $a$;

(iii) there is a section $\phi$ of $\tilde{\mathscr{Q}}_{1}(V ; \omega)$ over a neighborhood of a such that $\omega^{\prime}=\omega^{u_{0}}$, with $u_{0}=\mathscr{D} \phi$.

(iv) there is a local diffeomorphism $f: X \rightarrow X$ over $Y$ defined on a neighborhood of $a$ such that $f(a)=a$ and $f^{*} \omega=\omega^{\prime}$.

Proof. (ii) $\Rightarrow$ (i): Proposition 23.1 (i) shows that $\omega^{u_{0}}$ is non-singular; the implication (iii) $\Rightarrow$ (iv) of Proposition 22.6 gives us the relation (22.29), and so $d_{X / Y} \omega^{u_{0}}=0$.

(i) $\Rightarrow$ (iv) is given by Proposition 23.3 (i). 
(iv) $\Rightarrow$ (iii): According to Proposition 23.2, there is a section $u_{0}$ of $\left(T^{*} \otimes\right.$ $\left.J_{0}(V)\right)^{\wedge}$ such that $\omega^{\prime}=\omega^{u_{0}}$ over a neighborhood of $a$; assertion (iii) is given by the implication (i) $\Rightarrow$ (ii) of Proposition 22.6.

(iii) $\Rightarrow$ (ii): Since $Q_{1}(V ; \omega)$ is a formally integrable finite form of $J_{1}(V ; \omega)$ by Proposition 23.3 (ii), the implication (ii) $\Rightarrow$ (iii) of Proposition 22.6 tells us that assertion (ii) holds.

The proof of the implication (ii) $\Rightarrow$ (iii) of the above proposition gives us the more precise statement:

Corollary 23.1. Let $\omega$ be a volume (resp. symplectic) form along the fibers of $\rho$ and let $a \in X$. If $u$ is a section of $\left(T^{*} \otimes J_{1}(V ; \omega)\right)^{\wedge}$ satisfying $\mathfrak{D}_{1} u=0$, there exists a section $\phi$ of $\tilde{\mathscr{Q}}_{1}(V ; \omega)$ over a neighborhood of a such that $\mathfrak{D} \phi=\pi_{0} u$.

Since $Q_{1}(V ; \omega)$ is a formally integrable and integrable finite form of the Lie equation $J_{1}(V ; \omega)$ by Proposition 23.3 (ii), Corollary 23.1 together with Proposition 22.7 (iii), or the implication (ii) $\Rightarrow$ (iv) of Proposition 23.4 together with Proposition 22.8 gives us:

Theorem 23.1. Let $\omega$ be a volume or a symplectic form along the fibers of $\rho: X \rightarrow Y$. Then for all $m \geqslant 1, a \in X$, we have

$$
\bar{H}^{1}\left(Q_{1}(V ; \omega)\right)_{m, a}=0, \quad \tilde{H}^{1}\left(J_{1}(V ; \omega)\right)_{a}=0 .
$$

The existence of the local coordinates for $X$ satisfying the conditions with respect to $\omega$ described in the proof of Proposition 23.3 (i) shows that the sequence (22.36) is exact for $x \in X$ and $1 \leqslant k \leqslant \infty$; therefore $J_{1}(T ; \omega)$ is a formally transitive and formally integrable $\rho$-projectable Lie equation whose $k$ th prolongation is $J_{k+1}(T ; \omega)$. From $(22.32)$, we deduce that $J_{\infty}(V ; \omega)_{x}$ is a closed ideal of $J_{\infty}(T ; \omega)_{x}$, for $x \in X$.

Let $\{\omega\}$ be the sub-bundle of $\wedge^{r} V^{*}$ generated by the section $\omega$ and let $\varphi: \wedge^{r} V^{*} \rightarrow\{\omega\}$ be a projection of $\wedge^{r} V^{*}$ onto this sub-bundle. Let $E^{\prime}$ denote the quotient bundle $\wedge^{r} V^{*} /\{\omega\}$ and $\psi: \wedge^{r} V^{*} \rightarrow E^{\prime}$ be the natural projection. For $k \geqslant 2$, let

$$
\Phi_{k}(\omega): J_{k}(T ; \rho) \rightarrow J_{k-1}\left(E^{\prime}\right) \oplus J_{k-2}\left(V^{*}\right)
$$

be the morphism of vector bundles sending $j_{k}(\xi)(x)$, with $\xi \in \mathcal{T}_{\rho, x}$, into $\left(j_{k-1}\left(\psi D_{\omega} \xi\right)(x), j_{k-1}\left(d_{X / Y} f\right)(x)\right)$, where $f$ is the unique element of $\theta_{X, x}$ satisfying $\varphi D_{\omega} \xi=f \omega$. The kernel $J_{k}^{\prime}(T ; \omega)$ of $\Phi_{k}(\omega)$ consists of all $k$-jets $j_{k}(\xi)(x)$, with $\xi \in \mathcal{T}_{\rho, x}$, for which there is a real-valued function $f$ on $Y$ such that

$$
j_{k-1}\left(\mathcal{L}(\xi) \omega-\rho^{*} f \cdot \omega\right)(x)=0 ;
$$

then

$$
J_{k}(T ; \omega) \subset J_{k}^{\prime}(T ; \omega)
$$


We set

$$
J_{k}^{\prime}(V ; \omega)=J_{k}(V) \cap J_{k}^{\prime}(T ; \omega),
$$

for $k \geqslant 2$. By means of the morphisms $\Phi_{k}(\omega)$, we see that, if $J_{2}^{\prime}(T ; \omega)$ (resp. $\left.J_{2}^{\prime}(V ; \omega)\right)$ is a vector bundle, then its $k$ th prolongation is $J_{k+2}^{\prime}(T ; \omega)$ (resp. $\left.J_{k+2}^{\prime}(V ; \omega)\right)$. If $\xi_{0}$ is one of the vertical vector fields (23.2) and $f$ is a real-valued function on $Y$, then $j_{k}\left(\rho^{*} f \cdot \xi_{0}\right)$ is a section of $J_{k}^{\prime}(V ; \omega)$, for $k \geqslant 2$. Using (23.3), it is easily verified that

$$
\begin{aligned}
& {\left[J_{k+1}^{\prime}(T ; \omega), J_{k+1}^{\prime}(T ; \omega)\right] \subset J_{k}^{\prime}(T ; \omega),} \\
& {\left[J_{k+1}^{\prime}(T ; \omega), J_{k+1}^{\prime}(V ; \omega)\right] \subset J_{k}(V ; \omega),}
\end{aligned}
$$

for $k \geqslant 2$. For $x \in X$ and $k \geqslant 2$, the mapping

$$
J_{k}^{\prime}(V ; \omega)_{x} \rightarrow J_{k-1}(1 ; Y)_{\rho(x)},
$$

sending $j_{k}(\xi)(x)$, with $\xi \in \mathscr{V}_{x}$, into $j_{k-1}(f)(\rho(x))$, where $f$ is a real-valued function on $Y$ such that (23.3) holds, is easily seen to be well-defined. Because of the existence of the vector fields (23.2), the sequence

$$
0 \rightarrow J_{k}(V ; \omega) \rightarrow J_{k}^{\prime}(V ; \omega) \rightarrow \rho^{-1} J_{k-1}(1 ; Y) \rightarrow 0,
$$

induced by these mappings, is exact. We immediately deduce from this fact and (23.4) that $J_{2}^{\prime}(V ; \omega)$ is a formally integrable Lie equation whose $k$ th prolongation is $J_{k+2}^{\prime}(V ; \omega)$. For $x \in X$, we consider $J_{\infty}(1 ; Y)_{\rho(x)}$ as an abelian Lie algebra, and we write

$$
J_{\infty}^{\prime 0}(V ; \omega)_{x}=J_{\infty}^{0}(V)_{x} \cap J_{\infty}^{\prime}(V ; \omega)_{x} .
$$

If $\xi_{0}$ is one of the vector fields (23.2) defined on a neighborhood of $x$ satisfying $\xi_{0}(x)=0$, the morphism of Lie algebras

$$
J_{\infty}(1 ; Y)_{\rho(x)} \rightarrow J_{\infty}^{\prime 0}(V ; \omega)_{x}
$$

sending $j_{\infty}(f)(\rho(x))$ into $j_{\infty}\left(\rho^{*} f \cdot \xi_{0}\right)(x)$, where $f \in \Theta_{Y, \rho(x)}$, is a splitting of the exact sequence of Lie algebras

$$
0 \rightarrow J_{\infty}(V ; \omega)_{x} \rightarrow J_{\infty}^{\prime}(V ; \omega)_{x} \rightarrow J_{\infty}(1 ; Y)_{\rho(x)} \rightarrow 0 ;
$$

clearly the image of this mapping is a closed abelian subalgebra of $J_{\infty}^{\prime 0}(V ; \omega)_{x}$ which is a complement of $J_{\infty}(V ; \omega)_{x}$ in $J_{\infty}^{\prime}(V ; \omega)_{x}$, and so the quotient $J_{\infty}^{\prime}(V ; \omega)_{x} / J_{\infty}(V ; \omega)_{x}$ is abelian. Moreover the existence of the local coordinates for $X$ satisfying the conditions with respect to $\omega$ described in the proof of Proposition 23.3 (i) shows that the sequence

$$
0 \rightarrow J_{k}^{\prime}(V ; \omega)_{x} \rightarrow J_{k}^{\prime}(T ; \omega)_{x} \stackrel{\rho}{\rightarrow} J_{k}\left(T_{Y} ; Y\right)_{\rho(x)} \rightarrow 0
$$


is exact, for $x \in X$ and $2 \leqslant k \leqslant \infty$; therefore by (23.4) we see that $J_{2}^{\prime}(T ; \omega)$ is a formally transitive and formally integrable Lie equation whose $k$ th prolongation is $J_{k+2}^{\prime}(T ; \omega)$. From (23.5), we deduce that

$$
\left[\tilde{J}_{2}^{\prime}(\mathscr{T} ; \omega), J_{1}(\mathfrak{V} ; \omega)\right] \subset J_{1}(\mathscr{V} ; \omega),
$$

if $\tilde{J}_{2}^{\prime}(T ; \omega)=\nu^{-1} J_{2}^{\prime}(T ; \omega)$, and that $J_{\infty}^{\prime}(V ; \omega)_{x}$ and $J_{\infty}(V ; \omega)_{x}$ are closed ideals of the transitive Lie algebra $J_{\infty}^{\prime}(T ; \omega)_{x}$, for $x \in X$; moreover $J_{\infty}(T ; \omega)_{x}$ is a closed transitive subalgebra of $J_{\infty}^{\prime}(T ; \omega)_{x}$. With respect to the structure of analytic manifold on an open set $U \subset X$ determined by a local coordinate on $U$ satisfying the conditions with respect to $\omega$ described in the proof of Proposition 23.3 (i), the section $\omega$ is analytic and the Lie equations $J_{1}(V ; \omega)$ and $J_{2}^{\prime}(T ; \omega)$ are analytic and satisfy (23.6); hence for $x \in X$ and $j>0$, we have

$$
\begin{aligned}
& H^{j}\left(J_{\infty}^{\prime}(T ; \omega)_{x}, J_{\infty}(V ; \omega)_{x}\right)=H^{j}\left(J_{1}(V ; \omega)\right)_{x}=0, \\
& \tilde{H}^{1}\left(J_{\infty}^{\prime}(T ; \omega)_{x}, J_{\infty}(V ; \omega)_{x}\right)=\tilde{H}^{1}\left(J_{1}(V ; \omega)\right)_{x}=0,
\end{aligned}
$$

by Proposition 22.4 and Theorem 23.1. According to Proposition 22.4, the characteristic variety $\widetilde{V}_{x}\left(J_{1}(V ; \omega), \mathbf{C}\right)$ of $J_{1}(V ; \omega)$ is equal to $T_{x}^{*} \otimes \mathbf{C}$ if $q>1$, and to $\rho_{x}^{*} T_{Y, \rho(x)} \otimes \mathbf{C}$ if $q=1$ and $\omega$ is a volume form. Hence by Theorem 16.4 (i), the characteristic variety $\mathcal{V}\left(J_{\infty}^{\prime}(T ; \omega)_{x}, J_{\infty}(V ; \omega)_{x}, \mathbf{C}\right)$ is equal to the image of the injective mapping

$$
\pi_{0}^{*} \circ \nu^{*-1} \otimes \mathrm{id}: T_{x}^{*} \otimes \mathbf{C} \rightarrow J_{\infty}^{\prime}(T ; \omega)_{x}^{*} \otimes \mathbf{C}
$$

if $q>1$, or to the image of $\rho_{x}^{*} T_{Y, \rho(x)}^{*} \otimes \mathbf{C}$ under this mapping if $q=1$.

If the manifold $Y$ consists of just one point, then $V=T$ and $\omega$ is either a volume or a symplectic form on $X$. Applying the above results to $\omega$, we see that $J_{1}(T ; \omega)$ and $J_{2}^{\prime}(T ; \omega)$ are formally transitive and formally integrable Lie equations on $X$, and that $J_{\infty}(T ; \omega)_{x}$ is a closed ideal of $J_{\infty}^{\prime}(T ; \omega)_{x}$ of codimension one, for $x \in X$; moreover there is a closed abelian subalgebra of $J_{\infty}^{\prime 0}(T ; \omega)_{x}$ of dimension one which is a complement to $J_{\infty}(T ; \omega)_{x}$ in $J_{\infty}^{\prime}(T ; \omega)_{x}$. If $\omega$ is a volume form on $X$, the solutions of $J_{1}(T ; \omega)$ (resp. $J_{2}^{\prime}(T ; \omega)$ ) are the vector fields with zero-divergence (resp. with locally constant divergence), $J_{\infty}(T ; \omega)_{x}$ is the Lie algebra of formal vector fields with zero-divergence, and the local diffeomorphisms solutions of $Q_{1}(T ; \omega)$ are the volume preserving diffeomorphisms of $X$. If $\omega$ is a symplectic form on $X$, the solutions of $J_{1}(T ; \omega)$ (resp. $\left.J_{2}^{\prime}(T ; \omega)\right)$ are the symplectic vector fields (resp. the vector fields $\xi$ satisfying $\mathcal{L}(\xi) \omega=c \omega$, where $c$ is a locally constant function depending on $\xi), J_{\infty}(T ; \omega)_{x}$ is the Lie algebra of formal symplectic vector fields, and the local diffeomorphisms solutions of $Q_{1}(T ; \omega)$ are the symplectic diffeomorphisms of $X$. If $n \geqslant 2$, in either case the transitive Lie algebra $J_{\infty}(T ; \omega)_{x}$ is non-abelian, simple and infinite-dimensional; moreover if $\operatorname{Der}\left(J_{\infty}(T ; \omega)_{x}\right)$ denotes the Lie algebra 
of derivations of $J_{\infty}(T ; \omega)_{x}$, according to [7] the mapping

$$
J_{\infty}^{\prime}(T ; \omega)_{x} \rightarrow \operatorname{Der}\left(J_{\infty}(T ; \omega)_{x}\right),
$$

sending $\xi$ into the derivation $\operatorname{ad}(\xi)$ of $J_{\infty}(T ; \omega)_{x}$, is an isomorphism of Lie algebras (see [6, Corollary 2.2]).

We no longer assume that $Y$ is a point. Let $b \in Y$ and let $Z$ be the submanifold $X_{b}$ of $X$. We consider the volume or symplectic form $\omega_{Z}$, restriction of $\omega$ to $Z$. If $a \in Z$, a local coordinate on a neighborhood of $a$ satisfying the conditions with respect to $\omega$ described in the proof of Proposition 23.3 (i) determines a local isomorphism $\varphi: Y \times Z \rightarrow X$ of fibered manifolds over $Y$ defined on a neighborhood of $(b, a)$ such that $\varphi(b, a)=a$ and $\tilde{\omega}_{Z}=\varphi^{*}(\omega)$. If $y \in Y, z \in Z$ with $\varphi(y, z)=x$, the mapping $\varphi$ induces an isomorphism (21.4) of transitive Lie algebras; under this isomorphism, it is easily seen that the image of the semi-direct product

$$
M^{\prime}=\left(J_{\infty}^{\prime}\left(T_{Z} ; \omega_{Z}\right)_{z} \hat{\otimes} F_{y}\right) \oplus J_{\infty}\left(T_{Y} ; Y\right)_{y}
$$

is the transitive subalgebra $J_{\infty}^{\prime}(T ; \omega)_{x}$ of $J_{\infty}(T ; \rho)_{x}$, that the images of the closed ideals $J_{\infty}^{\prime}\left(T_{z} ; \omega_{z}\right)_{z} \hat{\otimes} F_{y}$ and $J_{\infty}\left(T_{z} ; \omega_{z}\right)_{z} \hat{\otimes} F_{y}$ of $M^{\prime}$ are the closed ideals $J_{\infty}^{\prime}(V ; \omega)_{x}$ and $J_{\infty}(V ; \omega)_{x}$ of $J_{\infty}^{\prime}(T ; \omega)_{x}$ respectively, and that the image of the closed subalgebra

$$
M=\left(J_{\infty}\left(T_{Z} ; \omega_{Z}\right)_{z} \hat{\otimes} F_{y}\right) \oplus J_{\infty}\left(T_{Y} ; Y\right)_{y}
$$

of $M^{\prime}$ is the closed subalgebra $J_{\infty}(T ; \omega)_{x}$ of $J_{\infty}^{\prime}(T ; \omega)_{x}$. If the dimension $q$ of $Z$ is $>1$, since $J_{\infty}\left(T_{Z} ; \omega_{Z}\right)_{z}$ is a non-abelian and simple Lie algebra, $J_{\infty}\left(T_{Z} ; \omega_{Z}\right)_{z} \hat{\otimes} F_{y}$ is a non-abelian minimal closed ideal of $M^{\prime}$ or of $M$; therefore $J_{\infty}(V ; \omega)_{x}$ is a non-abelian minimal closed ideal of $J_{\infty}^{\prime}(T ; \omega)_{x}$ or of $J_{\infty}(T ; \omega)_{x}$ whenever $q>1$. If $\omega_{Z}$ is a volume form on a one-dimensional manifold $Z$, then $J_{\infty}\left(T_{Z} ; \omega_{Z}\right)_{z}$ is abelian, and therefore so are the ideals $J_{\infty}\left(T_{Z} ; \omega_{Z}\right)_{z} \hat{\otimes} F_{y}$ and $J_{\infty}(V ; \omega)_{x}$. Thus

$$
\begin{gathered}
M^{\prime} \supset J_{\infty}^{\prime}\left(T_{z} ; \omega_{Z}\right)_{z} \hat{\otimes} F_{y} \supset J_{\infty}\left(T_{Z} ; \omega_{Z}\right)_{z} \hat{\otimes} F_{y} \supset 0, \\
M \supset J_{\infty}\left(T_{Z} ; \omega_{z}\right)_{z} \hat{\otimes} F_{y} \supset 0, \\
J_{\infty}^{\prime}(T ; \omega)_{x} \supset J_{\infty}^{\prime}(V ; \omega)_{x} \supset J_{\infty}(V ; \omega)_{x} \supset 0, \\
J_{\infty}(T ; \omega)_{x} \supset J_{\infty}(V ; \omega)_{x} \supset 0
\end{gathered}
$$

are Jordan-Hölder sequences for the transitive Lie algebras $M^{\prime}, M$, $J_{\infty}^{\prime}(T ; \omega)_{x}, J_{\infty}(T ; \omega)_{x}$. If $S_{z}$ is a closed abelian subalgebra of $J_{\infty}^{\prime 0}\left(T_{Z} ; \omega_{Z}\right)_{z}$ which is a complement to $J_{\infty}\left(T_{Z} ; \omega_{Z}\right)_{z}$ in $J_{\infty}^{\prime}\left(T_{Z} ; \omega_{Z}\right)_{z}$, then

$$
\left(S_{z} \otimes F_{y}\right) \oplus J_{\infty}\left(T_{Y} ; Y\right)_{y}
$$


is a closed subalgebra of $M^{\prime}$ which is a complement to $J_{\infty}\left(T_{Z} ; \omega_{Z}\right)_{z} \hat{\otimes} F_{y}$ in $M^{\prime}$. From the commutativity of diagram (21.6), we deduce that the characteristic variety $\mathscr{V}\left(M^{\prime}, J_{\infty}\left(T_{Z} ; \omega_{Z}\right)_{z} \hat{\otimes} F_{y}, \mathrm{C}\right)$ of the closed ideal $J_{\infty}\left(T_{Z} ; \omega_{Z}\right)_{z} \hat{\otimes} F_{y}$ of $M^{\prime}$ over $\mathrm{C}$ is equal to the image of the injective mapping (21.7), with $L=$ $J_{\infty}^{\prime}\left(T_{Z} ; \omega_{Z}\right)_{z}$, if the dimension $q$ of $Z$ is $>1$, or to the image of $T_{Y, y}^{*} \otimes \mathbf{C}$ under this mapping if $q=1$ and $\omega_{Z}$ is a volume form.

We restate some of these results in

Proposition 23.5. Let $\omega$ be a volume or a symplectic form along the fibers of $\rho: X \rightarrow Y$. Let $x \in X$ with $y=\rho(x)$, and $Z=\rho^{-1}(x)$. If $\omega_{Z}$ is the volume or symplectic form on $Z$, restriction of $\omega$ to $Z$, then $J_{\infty}^{\prime}\left(T_{Z} ; \omega_{Z}\right)_{x}$ and $J_{\infty}\left(T_{Z} ; \omega_{Z}\right)_{x}$ are transitive subalgebras of $J_{\infty}\left(T_{Z} ; Z\right)_{x}$, and $J_{\infty}\left(T_{Z} ; \omega_{Z}\right)_{x}$ is a closed ideal of codimension one of $J_{\infty}^{\prime}\left(T_{Z} ; \omega_{Z}\right)_{x}$. Moreover, there is an isomorphism of transitive Lie algebras

$$
\left(J_{\infty}^{\prime}\left(T_{Z} ; \omega_{Z}\right)_{x} \hat{\otimes} F_{y}\right) \oplus J_{\infty}\left(T_{Y} ; Y\right)_{y} \rightarrow J_{\infty}^{\prime}(T ; \omega)_{x}
$$

mapping the closed ideals $J_{\infty}^{\prime}\left(T_{z} ; \omega_{z}\right)_{x} \hat{\otimes} F_{y}$ and $J_{\infty}\left(T_{z} ; \omega_{Z}\right) \hat{\otimes} F_{y}$ of the semidirect product onto the closed ideals $J_{\infty}^{\prime}(V ; \omega)_{x}$ and $J_{\infty}(V ; \omega)_{x}$ of $J_{\infty}^{\prime}(T ; \omega)_{x}$, and the closed subalgebra

$$
\left(J_{\infty}\left(T_{Z} ; \omega_{Z}\right)_{x} \hat{\otimes} F_{y}\right) \oplus J_{\infty}\left(T_{Y} ; Y\right)_{y}
$$

of the semi-direct product onto the closed subalgebra $J_{\infty}(T ; \omega)_{x}$ of $J_{\infty}^{\prime}(T ; \omega)_{x}$.

Let $\omega_{Z}$ be a volume or a symplectic form on a manifold $Z$. Let $\rho: X \rightarrow Y$ be the fibered manifold $\mathrm{pr}_{1}: Y \times Z \rightarrow Y$ and $\omega$ be the volume or symplectic form $\tilde{\omega}_{Z}$ along the fibers of $\rho$ determined by $\omega_{Z}$. We have the decompositions (22.35) and

$$
J_{k}^{\prime}(T ; \omega)=J_{k}^{\prime}(V ; \omega) \oplus J_{k}\left(V^{\prime} ; \rho\right)
$$

and

$$
\begin{aligned}
& J_{\infty}^{\prime}(T ; \omega)_{x}=J_{\infty}^{\prime}(V ; \omega)_{x} \oplus \sigma_{x}\left(J_{\infty}\left(T_{Y} ; Y\right)_{y}\right), \\
& J_{\infty}(T ; \omega)_{x}=J_{\infty}(V ; \omega)_{x} \oplus \sigma_{x}\left(J_{\infty}\left(T_{Y} ; Y\right)_{y}\right),
\end{aligned}
$$

for $x=(y, z) \in Y \times Z$. Therefore $J_{\infty}^{\prime}(V ; \omega)_{x}$ and $J_{\infty}(V ; \omega)_{x}$ are $J_{\infty}\left(T_{Y} ; Y\right)_{y}{ }^{-}$ submodules of $J_{\infty}(V)_{x}$, and the restrictions

$$
\begin{aligned}
& \lambda: J_{\infty}^{\prime}\left(T_{z} ; \omega_{Z}\right)_{z} \hat{\otimes} F_{y} \rightarrow J_{\infty}^{\prime}(V ; \omega)_{x}, \\
& \lambda: J_{\infty}\left(T_{z} ; \omega_{z}\right)_{z} \hat{\otimes} F_{y} \rightarrow J_{\infty}(V ; \omega)_{x}
\end{aligned}
$$

of the mapping (21.3) are isomorphisms of $J_{\infty}\left(T_{Y} ; Y\right)_{y}$-modules. 
From (23.7) and Proposition 23.5, we deduce the following:

Theorem 23.2. If $\omega_{Z}$ is a volume or a symplectic form on a manifold $Z$, then for $z \in Z$ and $y \in Y$, we have

$$
H^{j}\left(\left(J_{\infty}^{\prime}\left(T_{Z} ; \omega_{Z}\right)_{z} \hat{\otimes} F_{y}\right) \oplus J_{\infty}\left(T_{Y} ; Y\right)_{y}, J_{\infty}\left(T_{Z} ; \omega_{Z}\right)_{z} \hat{\otimes} F_{y}\right)=0
$$

for $j>0$, and

$$
\tilde{H}^{1}\left(\left(J_{\infty}^{\prime}\left(T_{Z} ; \omega_{Z}\right)_{z} \hat{\otimes} F_{y}\right) \oplus J_{\infty}\left(T_{Y} ; Y\right)_{y}, J_{\infty}\left(T_{Z} ; \omega_{Z}\right)_{z} \hat{\otimes} F_{y}\right)=0 .
$$

If we take $Y$ to be a point and $Z=X$ in Theorem 23.2, we obtain:

Corollary 23.2. If $\omega$ is a volume or a symplectic form on $X$, then for $x \in X$ and $j>0$, we have

$$
H^{j}\left(J_{\infty}(T ; \omega)_{x}\right)=0, \quad \tilde{H}^{1}\left(J_{\infty}(T ; \omega)_{x}\right)=0 .
$$

\section{Finite-dimensional Lie algebras with parameters}

In this section, we consider a third type of non-singular sections of the vector bundles $\wedge^{r} V^{*} \otimes_{X} E$ and the corresponding Lie equations.

Assume that $\rho: X \rightarrow Y$ is a bundle $G$ of Lie groups over $Y$. The Lie algebra $g$ of $G$ is the vector bundle over $Y$ whose fiber $\mathfrak{g}_{y}$ at $y \in Y$ is $V_{I(y)}(G)$, where $I(y)$ is the identity element of $G_{y}$. We consider the bracket on $g$ as a morphism of vector bundles

$$
\mathfrak{g} \otimes \mathfrak{g} \rightarrow \mathfrak{g}
$$

which, when restricted to the fiber $\mathfrak{g}_{y}$, is the usual bracket defined in terms of right-invariant vector fields on $G_{y}$. The Maurer-Cartan forms of $G$

$$
\omega: V \rightarrow \mathfrak{g}, \quad \sigma: V \rightarrow \mathfrak{g}
$$

are defined by

$$
\langle\xi, \omega\rangle=g^{-1} \cdot \xi, \quad\langle\xi, \sigma\rangle=\xi \cdot g^{-1},
$$

for $\xi \in V_{g}$; if $g \in G$ and $\rho(g)=y$, the restrictions of $\omega$ and $\sigma$ to $V_{g}=T_{g}\left(G_{y}\right)$ are respectively the left-invariant and right-invariant Maurer-Cartan forms of the Lie group $G_{y}$. They define isomorphisms of vector bundles

$$
\omega: V \rightarrow \rho^{-1} \mathfrak{g}, \quad \sigma: V \rightarrow \rho^{-1} \mathfrak{g}
$$


over $Y$, and we consider $\omega$ and $\sigma$ as non-singular sections of $V^{*} \otimes_{X} \mathfrak{g}$. Moreover $\omega$ satisfies the equation

$$
d_{X / Y} \omega-\frac{1}{2}[\omega, \omega]=0,
$$

where the bracket is given by (4.1) with $g$ endowed with the above bracket.

The bracket on $g$ induces a structure of Lie algebra on $\Gamma(Y, \mathfrak{g})$. Let

$$
\iota: \Gamma(Y, \mathfrak{g}) \rightarrow \Gamma(X, V)
$$

be the homomorphism of Lie algebras sending $\xi$ into the vertical vector field $\iota(\xi)$ on $X$ whose restriction to $X_{y}$ is the right-invariant vector field on $G_{y}$ whose value at $I(y)$ is $\xi(y)$, for all $y \in Y$. Then

$$
\langle\iota(\xi), \sigma\rangle(g)=\xi(\rho(g)),
$$

for $g \in G$. Let

$$
\text { Ad: } V^{*} \otimes_{X} \mathfrak{g} \rightarrow V^{*} \otimes_{X} \mathfrak{g}
$$

be the isomorphism of vector bundles over $X$ sending $u \in V_{g}^{*} \otimes \mathfrak{g}_{y}$, with $g \in G$ and $\rho(g)=y$, into (id $\otimes \operatorname{Ad} g$ ) $u$. Then

$$
\sigma=\operatorname{Ad}(\omega) \text {. }
$$

Lemma 24.1. If $\eta$ is a vertical vector field on $X$ whose restriction to $X_{y}$ is a left-invariant vector field on $G_{y}$ for all $y \in Y$, and $\xi$ is an arbitrary vertical vector field on $X$, then we have

$$
\left\langle\eta, d_{X / Y}\langle\xi, \sigma\rangle\right\rangle=\langle[\eta, \xi], \sigma\rangle,
$$

as sections of $\rho^{-1} \mathrm{~g}$ over $X$, and

$$
\operatorname{Ad}(\mathcal{L}(\xi) \omega)=d_{X / Y}\langle\xi, \sigma\rangle .
$$

Proof. There is a one-parameter family of sections $g_{t}$ of $G$ over $Y$ such that $g_{t}(y)$ is a one-parameter subgroup of $G_{y}$ for all $y \in Y$, and

$$
\eta(g)=\left.\frac{d}{d t} g \cdot g_{t}(y)\right|_{t=0},
$$

for $g \in G_{y}, y \in Y$. If $\varphi_{t}: G \rightarrow G$ is the diffeomorphism sending $g \in G_{y}$ into $g \cdot g_{t}(y)$, with $y \in Y$, then $\eta=d \varphi_{t} /\left.d t\right|_{t=0}$, and if $\xi$ is a vertical vector field on $X$,

$$
\begin{aligned}
{[\eta, \xi](g) } & =-\left.\frac{d}{d t} \varphi_{t *} \xi\left(\varphi_{t}^{-1}(g)\right)\right|_{t=0} \\
& =-\left.\frac{d}{d t} \xi\left(g \cdot g_{t}^{-1}(y)\right) \cdot g_{t}(y)\right|_{t=0}
\end{aligned}
$$


for $g \in G_{y}$. Thus

$$
\begin{aligned}
\langle[\eta, \xi], \sigma\rangle(g) & =-\left.\left(\frac{d}{d t} \xi\left(g \cdot g_{t}^{-1}(y)\right) \cdot g_{t}(y)\right)\right|_{t=0} \cdot g^{-1} \\
& =-\left.\frac{d}{d t} \xi\left(g \cdot g_{t}^{-1}(y)\right) \cdot\left(g_{t}(y) \cdot g^{-1}\right)\right|_{t=0} \\
& =-\left.\frac{d}{d t}\langle\xi, \sigma\rangle\left(g \cdot g_{t}^{-1}(y)\right)\right|_{t=0} \\
& =-\left.\frac{d}{d t}\langle\xi, \sigma\rangle\left(g \cdot g_{-t}(y)\right)\right|_{t=0} \\
& =\left.\frac{d}{d t}\langle\xi, \sigma\rangle\left(g \cdot g_{t}(y)\right)\right|_{t=0} \\
& =\left\langle\eta, d_{X / Y}\langle\xi, \sigma\rangle\right\rangle(g)
\end{aligned}
$$

and so (24.3) holds. On the other hand, $\langle\eta, \omega\rangle$ is a constant function on each fiber of $X$, and hence

$$
\langle\eta, \mathcal{L}(\xi) \omega\rangle=\mathcal{L}(\xi)\langle\eta, \omega\rangle+\langle[\eta, \xi], \omega\rangle=\langle[\eta, \xi], \omega\rangle .
$$

According to (24.2) and (24.3), we thus obtain

$$
\begin{aligned}
\langle\eta, \operatorname{Ad}(\mathcal{L}(\xi) \omega)\rangle & =\langle[\eta, \xi], \operatorname{Ad}(\omega)\rangle=\langle[\eta, \xi], \sigma\rangle \\
& =\left\langle\eta, d_{X / Y}\langle\xi, \sigma\rangle\right\rangle,
\end{aligned}
$$

from which we deduce (24.4).

We also write $E$ for $\mathfrak{g}$ and consider the differential operator $D_{\omega}: \mathfrak{V} \rightarrow$ $\mathcal{V}^{*} \otimes \mathcal{E}_{X}$ sending $\xi$ into $\mathcal{L}(\xi) \omega$. According to (24.1) and (24.4), the diagram

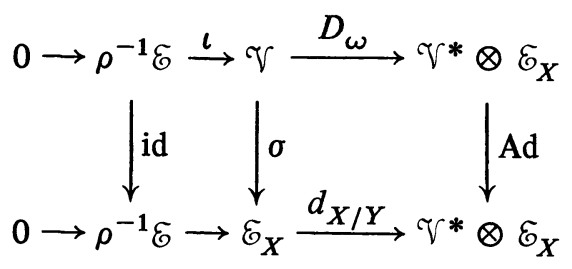

is commutative; since its vertical arrows are induced by isomorphisms of vector bundles and (22.5) is exact, its rows are exact. If $\xi$ is a vertical vector field on $X$, from (24.5) it follows that $\mathcal{L}(\xi) \omega=0$ if and only if $[\xi, \eta]=0$ for all vector fields $\eta$ satisfying the left-invariance condition of Lemma 24.1. It is easy to verify that this holds if and only if $\xi$ belongs to the image of $\iota$. We thus obtain a direct proof of the exactness of the top row of diagram (24.6). The hypotheses of Proposition 22.4 are satisfied with $r=1$ and $\tau=$ Ad. Therefore $J_{1}(V ; \omega)$ is a formally integrable Lie equation whose $k$ th prolongation is $J_{k+1}(V ; \omega)$, and $Q_{1}(V ; \omega)$ is a finite form of $J_{1}(V ; \omega)$ whose $k$ th prolongation 
is the fibered manifold $Q_{k+1}(V ; \omega)$; moreover $\pi_{0}\left(J_{1}(V ; \omega)\right)=J_{0}(V)$ and (22.21) holds. The solutions of $J_{1}(V ; \omega)$ over open subsets of $X$ whose fibers over $Y$ are connected are the vector fields of the form $\iota(\xi)$, with $\xi$ a section of $g$ over an open subset of $Y$. Furthermore, diagram (22.22) is commutative and exact, and $J_{k}\left(\sigma^{-1}\right)$ induces an isomorphism of vector bundles

$$
\iota_{k}: \rho^{-1} J_{k}(g ; Y) \rightarrow J_{k}(V ; \omega)
$$

over $X$; in fact, by (24.1) we see that $\iota_{k}$ sends $\left(g, j_{k}(\xi)(y)\right)$ into $j_{k}(\iota(\xi))(g)$, with $g \in G$ satisfying $\rho(g)=y$ and $\xi \in \Gamma(Y, \mathfrak{g})$. The bracket of $\mathfrak{g}$ induces a structure of linearly compact Lie algebra on $J_{\infty}(\mathrm{g} ; Y)_{y}$, for $y \in Y$, by setting

$$
\left[j_{\infty}(\xi)(y), j_{\infty}(\eta)(y)\right]=j_{\infty}([\xi, \eta])(y),
$$

for $\xi, \eta \in \Gamma(Y, \mathfrak{g})$. The mappings $\iota_{k}$ give rise to an isomorphism of linearly compact Lie algebras

$$
\iota_{g}: J_{\infty}(g ; Y)_{y} \rightarrow J_{\infty}(V ; \omega)_{g},
$$

for $g \in G$, with $\rho(g)=y$.

The bundle $\rho^{-1} G$ over $X$ is equal to $G \times{ }_{Y} G$ considered as a bundle over $X$ via the projection onto the first factor, and the sheaf of sections of $\rho^{-1} G$ over $X$ is $\mathcal{G}_{X}$. Sections of $\rho^{-1} G$ are precisely the graphs of local mappings $G \rightarrow G$ over $Y$. We identify a mapping $f: G \rightarrow G$ over $Y$ defined on an open set $U \subset G$ with its graph $\tilde{f}: U \rightarrow \rho^{-1} G$, and the $k$-jet $j_{k}(f)(x)$ with $j_{k}(\tilde{f})(x)$, for $x \in U$. We thus consider $Q_{k}(V)$ as an open fibered manifold of $J_{k}\left(\rho^{-1} G\right)$. If $f$ is a local mapping $G \rightarrow G$ over $Y$ defined on an open set $U$, let $\alpha(f): U \rightarrow G, \beta(f)$ : $U \rightarrow G$ be the mappings over $Y$ defined by

$$
\begin{aligned}
& \alpha(f)(a)=f(a) \cdot a^{-1}, \\
& \beta(f)(a)=f(a) \cdot a,
\end{aligned}
$$

for $a \in U$. Then

$$
\alpha(\beta(f))=f, \quad \beta(\alpha(f))=f,
$$

and so $\alpha, \beta$ determine isomorphisms of fibered manifolds over $U$

$$
\begin{aligned}
J_{k}(\alpha): J_{k}\left(\rho^{-1} G\right) & \rightarrow J_{k}\left(\rho^{-1} G\right), \\
J_{k}(\beta): J_{k}\left(\rho^{-1} G\right) & \rightarrow J_{k}\left(\rho^{-1} G\right),
\end{aligned}
$$

sending $j_{k}(f)(a)$ into $j_{k}(\alpha(f))(a)$ and $j_{k}(\beta(f))(a)$ respectively and satisfying

$$
J_{k}(\beta)=J_{k}(\alpha)^{-1} \text {. }
$$

Lemma 24.2. If $f: G \rightarrow G$ is a local mapping over $Y$, then

$$
\operatorname{Ad}\left(f^{*} \omega-\omega\right)=\alpha(f) * \omega
$$

as sections of $V^{*} \otimes_{X} \mathfrak{g}$. 
Proof. Let $\xi \in V_{a}$, with $a \in G$ and $\rho(a)=y$, and choose a one-parameter family $a_{t}$ of elements of $G_{y}$ such that $a_{0}=a$ and $d a_{t} /\left.d t\right|_{t=0}=\xi$. Then

$$
\left.\frac{d a_{t}^{-1}}{d t}\right|_{t=0}=-a^{-1} \cdot \xi \cdot a^{-1}
$$

and

$$
\begin{aligned}
\alpha(f)_{*} \xi & =\left.\frac{d}{d t} f\left(a_{t}\right) \cdot a_{t}^{-1}\right|_{t=0} \\
& =\left.\frac{d}{d t} f\left(a_{t}\right)\right|_{t=0} \cdot a^{-1}+\left.f(a) \cdot \frac{d a_{t}^{-1}}{d t}\right|_{t=0} \\
& =\left(f_{*} \xi\right) \cdot a^{-1}-f(a) \cdot a^{-1} \cdot \xi \cdot a^{-1}
\end{aligned}
$$

Thus

$$
\begin{aligned}
\left\langle\xi, \alpha(f)^{*} \omega\right\rangle & =\left\langle\alpha(f)_{*} \xi, \omega\right\rangle=\left(f(a) \cdot a^{-1}\right)^{-1} \cdot \alpha(f)_{*} \xi \\
& =a \cdot f(a)^{-1} \cdot \alpha(f)_{*} \xi \\
& =a \cdot f(a)^{-1} \cdot\left(f_{*} \xi\right) \cdot a^{-1}-\xi \cdot a^{-1} \\
& =\operatorname{Ad} a\left(\left\langle f_{*} \xi, \omega\right\rangle-\langle\xi, \omega\rangle\right) \\
& =\operatorname{Ad} a \cdot\left\langle\xi, f^{*} \omega-\omega\right\rangle,
\end{aligned}
$$

and so (24.7) holds.

If $g$ is a section of $G$ over an open set $U \subset Y$, let $\iota(g)$ be the mapping $G \rightarrow G$ defined on $\rho^{-1}(U)$ by

$$
\iota(g)(a)=g(\rho(a)) \cdot a,
$$

for $a \in \rho^{-1}(U)$. Then $\iota(g)$ is a local diffeomorphism of $G$ over $Y$ satisfying

$$
\alpha(\iota(g))=g \circ \rho ;
$$

the left-invariance of $\omega$ means that

$$
\iota(g) * \omega=\omega .
$$

Thus $\iota$ determines a morphism of fibered manifolds

$$
\iota_{k}: \rho^{-1} J_{k}(G ; Y) \rightarrow Q_{k}(V ; \omega)
$$

over $X$ sending $\left(a, j_{k}(g)(\rho(a))\right)$ into $j_{k}(\iota(g))(a)$, with $a \in \rho^{-1}(U)$.

We consider the sequence (4.6) (with $X=G$ ), where

$$
\begin{array}{ll}
\mathfrak{D}_{X / Y} f=f^{*} \omega, & \text { for } f \in \mathcal{G}_{X}, \\
\mathfrak{D}_{1, X / Y} v=d_{X / Y} v-\frac{1}{2}[v, v], & \text { for } v \in \mathcal{V}^{*} \otimes \mathcal{E}_{X}
\end{array}
$$


the bracket is given by (4.1) with $g$ endowed with the bracket considered above. From (24.8) and Lemma 24.2, we obtain the commutative diagram

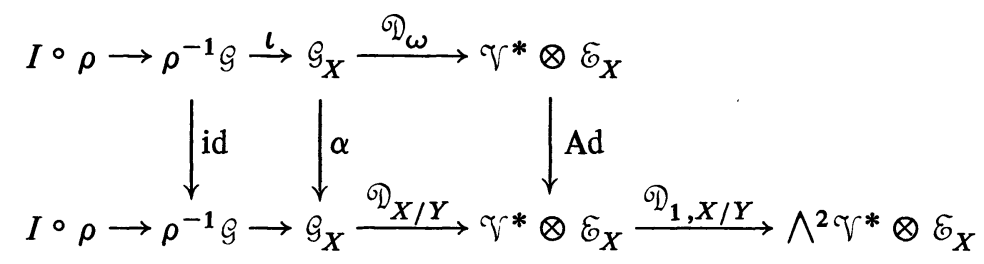

which is the finite form of diagram (24.6). Since its vertical arrows are bijective and its bottom row is exact by Proposition 4.1, its top row is also exact. Therefore the solutions of $Q_{1}(V ; \omega)$ over open subsets of $X$ whose fibers over $Y$ are connected are the local diffeomorphisms of $G$ of the form $\iota(g)$, with $g$ a section of $G$ over an open subset of $Y$.

If $E^{\prime}$ is a vector bundle over $G$, let $E^{\prime} \otimes_{\rho^{-1} G} V$ be the vector bundle whose fiber over $(a, b) \in G \times{ }_{Y} G$ is $E_{a}^{\prime} \otimes V_{b}$. If $a, b \in X$, with $\rho(a)=\rho(b)$, and if $u \in T_{a}^{*} \otimes V_{b}$, we denote by $u \pi \omega$ the element of $\left(V^{*} \otimes_{X} \mathfrak{g}\right)_{a}$ equal to the composition

$$
V_{a} \stackrel{u}{\rightarrow} V_{b} \stackrel{\omega}{\rightarrow} \mathfrak{g}_{\rho(b)},
$$

and by

$$
\tau(\omega): T^{*} \otimes_{\rho^{-1} G} V \rightarrow V^{*} \otimes_{X} \mathfrak{g}
$$

the mapping sending $u$ into $u \pi \omega$. Moreover, if

$$
\Delta_{k-1,1}: S^{k} T^{*} \rightarrow S^{k-1} T^{*} \otimes T^{*}
$$

is the morphism of vector bundles determined by

$$
\Delta_{k-1,1}\left(\xi_{1} \cdot \ldots \cdot \xi_{k}\right)=\sum_{j=1}^{k}\left(\xi \cdot \ldots \cdot \hat{\xi}_{j} \cdot \ldots \cdot \xi_{k}\right) \otimes \xi_{j},
$$

for $\xi_{1}, \ldots, \xi_{k} \in T^{*}$, where $\hat{\xi}_{j}$ indicates that $\xi_{j}$ is to be omitted, we denote by $\tau_{k-1}(\omega)$ the composition

$$
S^{k} T^{*} \otimes_{\rho^{-1} G} V \stackrel{\Delta_{k-1,1} \otimes \text { id }}{\longrightarrow} S^{k-1} T^{*} \otimes T^{*} \otimes_{\rho^{-1} G} V \stackrel{\text { id } \otimes \tau(\omega)}{\longrightarrow} S^{k-1} T^{*} \otimes V^{*} \otimes_{X} \mathrm{~g} ;
$$

then $\tau_{0}(\omega)=\tau(\omega)$. Since $\omega$ is a non-singular section of $V^{*} \otimes_{X} \mathfrak{g}$, from the exactness of (22.7) we deduce that the sequence

$$
0 \rightarrow S^{k} T_{Y, \rho(a)}^{*} \otimes V_{b} \stackrel{\rho_{a}^{*} \otimes \mathrm{id}}{\longrightarrow} S^{k} T_{a}^{*} \otimes V_{b} \stackrel{\tau_{k-1}(\omega)}{\longrightarrow}\left(S^{k-1} T^{*} \otimes V^{*} \otimes_{X} \mathfrak{g}\right)_{a}
$$

is exact, for $a, b \in X$ with $\rho(a)=\rho(b)$. 
We now study the morphisms

$$
\begin{aligned}
p_{k}\left(\mathfrak{D}_{X / Y}\right): J_{k+1}\left(\rho^{-1} G\right) & \rightarrow J_{k}\left(V^{*} \otimes_{X} \mathfrak{g}\right), \\
p_{k}\left(\mathfrak{D}_{\omega}\right): J_{k+1}\left(\rho^{-1} G\right) & \rightarrow J_{k}\left(V^{*} \otimes_{X} \mathfrak{g}\right)
\end{aligned}
$$

of fibered manifolds over $X$ determined by the first-order differential operators $\mathfrak{D}_{X / Y}$ and $\mathfrak{D}_{\omega}$ from $\mathcal{G}_{X}$ to $\mathscr{T} * \otimes \mathcal{E}_{X}$. We let $p_{-1}\left(\mathfrak{D}_{X / Y}\right)$ and $p_{-1}\left(\mathfrak{D}_{\omega}\right)$ be the projection $\mathrm{pr}_{1}: \rho^{-1} G \rightarrow X$ onto the first factor.

Proposition 24.1. For $k \geqslant 0$, the mapping $p_{k}\left(\mathfrak{D}_{X / Y}\right)\left(\right.$ resp. $\left.p_{k}\left(\mathfrak{D}_{\omega}\right)\right)$ is a morphism of affine bundles over $p_{k-1}\left(\mathfrak{D}_{X / Y}\right)\left(\right.$ resp. $\left.p_{k-1}\left(\mathfrak{D}_{\omega}\right)\right)$ whose associated morphism of vector bundles is induced by $\tau_{k}(\omega)$.

Proof. It suffices to show that

$$
p_{k}\left(\mathfrak{D}_{X / Y}\right)(\phi+u)=p_{k}\left(\mathfrak{D}_{X / Y}\right) \phi+\tau_{k}(\omega) u,
$$

for $\phi \in J_{k+1}\left(\rho^{-1} G\right), u \in S^{k} T^{*} \otimes_{\rho^{-1} G} V$ and $k \geqslant 0$, where the sum on the right-hand side is that of two elements of the vector bundle $J_{k}\left(V^{*} \otimes_{X} \mathfrak{g}\right)$. According to [9, Propositions 5.6 and 5.3], we need only verify this for $k=0$. Indeed, if $f, f^{\prime} \in \mathcal{G}_{X, a}, a \in X$, with $f(a)=f^{\prime}(a)=b$, and if $u \in T_{a}^{*} \otimes V_{b}$ satisfy

$$
j_{1}\left(f^{\prime}\right)(a)=j_{1}(f)(a)+u,
$$

then $f_{*}^{\prime}: V_{a} \rightarrow V_{b}$ is equal to $f_{*}+u_{\mid V_{a}}$. Hence we obtain

$$
\begin{aligned}
p_{0}\left(\mathfrak{D}_{X / Y}\right)\left(j_{1}\left(f^{\prime}\right)(a)\right) & =\left(f^{*} \omega\right)(a)=\omega \circ\left(f_{*}+u_{\mid V_{a}}\right) \\
& =\omega \circ f_{*}+u \pi \omega \\
& =p_{0}\left(\mathfrak{D}_{X / Y}\right)\left(j_{1}(f)(a)\right)+\tau_{0}(\omega) u,
\end{aligned}
$$

which gives us (24.12) for $k=0$.

From the commutativity of diagram (24.10), we deduce that of the diagram

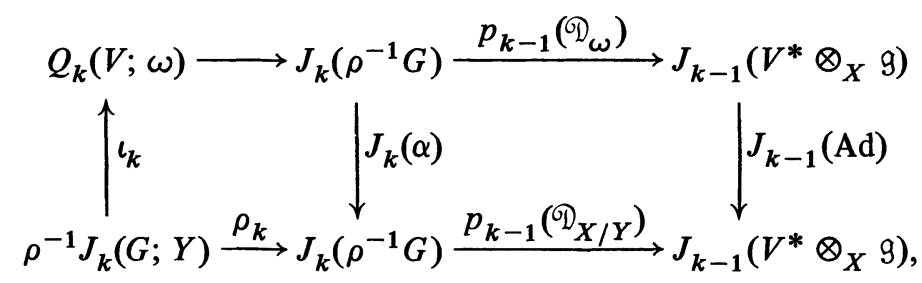

whose rows are complexes and whose mappings $J_{k}(\alpha)$ and $J_{k-1}(\mathrm{Ad})$ are the isomorphisms of fibered manifolds over $X$ determined by $\alpha$ and Ad respectively. We now verify that its bottom row is exact. 
Proposition 24.2. The set of elements $\phi$ of $J_{k}\left(\rho^{-1} G\right)$ satisfying $p_{k-1}\left(\mathfrak{D}_{X / Y}\right) \phi$ $=0$ is equal to $\rho_{k}\left(\rho^{-1} J_{k}(G ; Y)\right)$.

Proof. We proceed by induction on $k$. For $k=0$, the result is trivial with $J_{-1}\left(V^{*} \otimes_{X} \mathrm{~g}\right)=0$. Assume that it holds for $k-1$, with $k \geqslant 1$, and let $\phi=$ $j_{k}(f)(a)$ be an element of $J_{k}\left(\rho^{-1} G\right)$, where $f \in \mathcal{G}_{X, a}, a \in X$, satisfying $p_{k-1}\left(\mathfrak{D}_{X / Y}\right) \phi=0$. Then by our induction hypothesis, we may write $j_{k-1}(f)(a)=j_{k-1}(g \circ \rho)(a)$, for some section $g$ of $G$ over a neighborhood of $\rho(a)$. Since $\pi_{k-1} j_{k}(f)(a)=\pi_{k-1} j_{k}(g \circ \rho)(a)$, by [9, Proposition 5.1] there is an element $u \in S^{k} T_{a}^{*} \otimes V_{b}$, where $b=f(a)$, such that

$$
j_{k}(f)(a)=j_{k}(g \circ \rho)(a)+u \text {. }
$$

As

$$
p_{k-1}\left(\mathfrak{D}_{X / Y}\right) j_{k}(f)(a)=p_{k-1}\left(\mathfrak{D}_{X / Y}\right) j_{k}(g \circ \rho)(a)=0,
$$

we deduce from (24.14) and Proposition 24.1 that $\tau_{k-1}(\omega) u=0$. Since the sequence (24.11) is exact, there is an element $v \in S^{k} T_{Y, \rho(a)}^{*} \otimes V_{b}$ such that $\left(\rho_{a}^{*} \otimes \mathrm{id}\right) v=u$. By Proposition 22.1 and (24.14), we have

$$
\rho_{k}\left(a, j_{k}(g)(\rho(a))+v\right)=j_{k}(f)(a),
$$

concluding our proof.

Since $\rho_{k}$ is injective, by Proposition 24.2 and the commutativity of diagram (24.13), we see that (24.9) is an isomorphism of fibered manifolds and deduce the following:

Proposition 24.3. If $X$ is a bundle of Lie groups $G$ over $Y$ and $\omega$ is the Maurer-Cartan form of $G$ considered as a section of $V^{*} \otimes_{X} \mathfrak{g}$, then $Q_{1}(V ; \omega)$ is a formally integrable and integrable finite form of the formally integrable Lie equation $J_{1}(V ; \omega)$, whose kth prolongation is $Q_{k+1}(V ; \omega)$; moreover $(24.9)$ is an isomorphism of fibered manifolds over $X$.

Let

$$
\begin{aligned}
\gamma_{1}: T^{*} \otimes J_{0}(V) & \rightarrow V^{*} \otimes_{X} \mathfrak{g}, \\
\gamma_{2}: \wedge^{2} T^{*} \otimes J_{0}(V) & \rightarrow \wedge^{2} V^{*} \otimes_{X} \mathfrak{g}
\end{aligned}
$$

be the mappings sending $u$ and $v$ into $\omega^{u}$ and $\omega \circ \nu^{-1} \circ v_{\mid \wedge{ }^{2} V}=\left(\nu^{-1} \circ v_{\mid \wedge}{ }^{2} V\right) \pi \omega$ respectively. We have the identities

$$
\begin{gathered}
\gamma_{1} \mathfrak{D} \phi=\mathfrak{D}_{X / Y} \pi_{0} \phi, \quad \text { for } \phi \in \tilde{\mathcal{Q}}_{1}(V ; \omega), \\
\gamma_{2} \mathfrak{D}_{1} u=\mathfrak{D}_{1, X / Y}\left(\gamma_{1} \pi_{0} u\right), \quad \text { for } u \in \mathcal{T}^{*} \otimes J_{1}(\mathfrak{V} ; \omega) .
\end{gathered}
$$

Indeed, for $\phi \in \tilde{\mathcal{Q}}_{1}(V ; \omega)$, we have $f=\pi_{0} \phi \in \mathcal{G}_{X}$ and (24.15) is the relation (22.28). If $u \in \mathcal{T}^{*} \otimes J_{1}(\mathscr{V} ; \omega)$, then $\hat{u}=\left(\nu^{*-1} \otimes \nu^{-1}\right)\left(u_{\mid V}\right)$ is an element of 
$J_{0}(\mathscr{V})^{*} \otimes \tilde{J}_{1}(\mathfrak{V} ; \omega)$ and so

$$
\varrho_{v}(\hat{u})\left(\nu^{*-1} \omega\right)=0,
$$

where $u_{0}=\pi_{0} u$ and $v=u_{0 \mid V}$. Hence if we apply Proposition 22.3 to $\omega$ and $u_{\mid V}$, we obtain

$$
\begin{aligned}
\mathfrak{D}_{1, X / Y} \omega^{u_{0}} & =d_{X / Y} \omega^{u_{0}}-\frac{1}{2}\left[\omega^{u_{0}}, \omega^{u_{0}}\right] \\
& =\left(d_{X / Y}\right)^{u_{0}}+\left(\nu^{-1} \circ\left(\mathfrak{D}_{1} u\right)_{\mid \wedge{ }^{2} V}\right) \pi_{u_{0}} \omega-\frac{1}{2}([\omega, \omega])^{u_{0}} \\
& =\left(\mathfrak{D}_{1, X / Y} \omega\right)^{u_{0}}+\left(\nu^{-1} \circ\left(\mathfrak{D}_{1} u\right)_{\mid \wedge{ }^{2} V}\right) \pi \omega \\
& =\gamma_{2} \mathfrak{D}_{1} u,
\end{aligned}
$$

since $\mathfrak{D}_{1, X / Y} \omega=0$. These two identities imply that the diagram

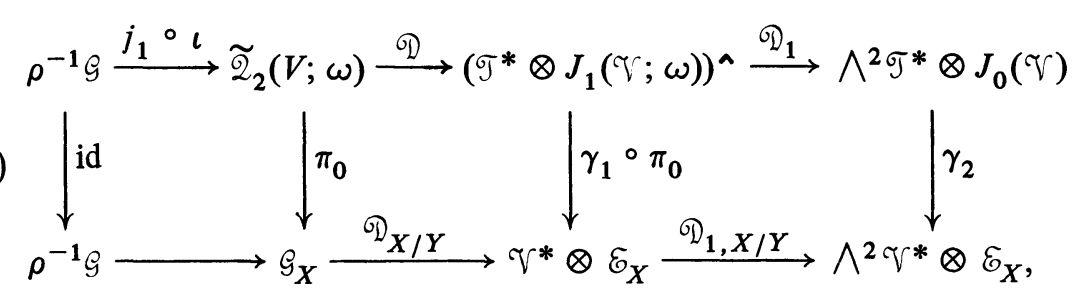

whose top row is the non-linear Spencer complex of $J_{1}(V ; \omega)$ and whose bottom row is the exact sequence (4.6) (see Proposition 4.1), is commutative.

The following proposition is the analogue of Propositions 23.2 and 23.4 for Maurer-Cartan forms. The equivalence of assertions (i) and (ii) of the following proposition shows that the mapping $\gamma_{1} \circ \pi_{0}: T^{*} \otimes J_{1}(V) \rightarrow V^{*} \otimes_{X} \mathrm{~g}$ induces a surjective mapping from $Z^{1}\left(J_{1}(V ; \omega)\right)_{a}$ to the set of germs at $a \in X$ of non-singular sections $v$ of $V^{*} \otimes_{X} \mathfrak{g}$ satisfying $\mathfrak{D}_{1, X / Y} v=0$.

Proposition 24.4. Let $\omega^{\prime}$ be a section of $V^{*} \otimes_{X} \mathfrak{g}$ over $X$. Then there is a section $u_{0}$ of $T^{*} \otimes J_{0}(V)$ over $X$ such that $\omega^{\prime}=\omega^{u_{0}}$. If $a \in X$, the following assertions are equivalent:

(i) $\omega^{\prime}$ is non-singular and satisfies the equation

$$
\mathfrak{D}_{1, X / Y} \omega^{\prime}=d_{X / Y} \omega^{\prime}-\frac{1}{2}\left[\omega^{\prime}, \omega^{\prime}\right]=0
$$

on a neighborhood of $a$;

(ii) there is a section $u$ of $\left(T^{*} \otimes J_{1}(V ; \omega)\right)^{\wedge}$ such that $\pi_{0} u=u_{0}$ and $\mathscr{D}_{1} u=0$ on a neighborhood of $a$;

(iii) there is a section $\phi$ of $\tilde{\mathscr{L}}_{1}(V ; \omega)$ over a neighborhood of a satisfying $\mathscr{D} \phi=u_{0}$

(iv) there is a local diffeomorphism $f: X \rightarrow X$ over $Y$ defined on a neighborhood of $a$ such that $f(a)=a$ and $f^{*} \omega=\omega^{\prime}$. 
Proof. Let $v$ be the section of $V^{*} \otimes V$ over $X$ equal to the composition

$$
V \stackrel{\omega^{\prime}}{\rightarrow} \rho^{-1} \mathfrak{g} \stackrel{\omega^{-1}}{\rightarrow} V,
$$

and let $u_{0}$ be a section of $T^{*} \otimes J_{0}(V)$ satisfying (id $\left.+\nu^{-1} \circ u_{0}\right)_{\mid V}=v$; then $\omega^{\prime}=\omega^{u_{0}}$.

(ii) $\Rightarrow$ (i): If $u$ is a section of $\left(T^{*} \otimes J_{1}(V ; \omega)\right)^{\wedge}$ satisfying $\pi_{0} u=u_{0}$ and $\mathfrak{D}_{1} u=0$, Proposition 23.1 (i) shows that $\omega^{u_{0}}$ is a non-singular section of $V^{*} \otimes_{X} \mathfrak{g}$. The relation (25.16) tells us that $\mathfrak{D}_{1, X / Y} \omega^{u_{0}}=0$; this fact can also be obtained as a consequence of the implication (iii) $\Rightarrow$ (ii) of Proposition 22.6.

(i) $\Rightarrow$ (iv): Taking $X=G$ in Proposition 4.1, we obtain a local mapping $f: X \rightarrow X$ over $Y$ defined on a neighborhood of $a$ satisfying $f(a)=a$ and $f^{*} \omega=\omega^{\prime}$. From Proposition 23.1 (ii), it follows that $f$ is $a$ local diffeomorphism on a neighborhood of $a$.

(iv) $\Rightarrow$ (iii) is given by the implication (i) $\Rightarrow$ (ii) of Proposition 22.6.

(iii) $\Rightarrow$ (ii): Since $Q_{1}(V ; \omega)$ is a formally integrable finite form of $J_{1}(V ; \omega)$ by Proposition 24.3, the implication (ii) $\Rightarrow$ (iii) of Proposition 22.6 tells us that assertion (ii) holds.

Since $Q_{1}(V ; \omega)$ is a formally integrable and integrable finite form of the Lie equation $J_{1}(V ; \omega)$ by Proposition 24.3 , the implication (ii) $\Rightarrow$ (iii) of Proposition 24.4 together with Proposition 22.7 (iii), or the implication (ii) $\Rightarrow$ (iv) of Proposition 24.4 together with Proposition 22.8 gives us:

Theorem 24.1. If $X$ is a bundle of Lie groups $G$ over $Y$ and $\omega$ is the Maurer-Cartan form of $G$ considered as a section of $V^{*} \otimes_{X} \mathfrak{g}$, then for all $m \geqslant 1$ and $a \in X$ we have

$$
\bar{H}^{1}\left(Q_{1}(V ; \omega)\right)_{m, a}=0, \quad \tilde{H}^{1}\left(J_{1}(V ; \omega)\right)_{a}=0 .
$$

If the manifold $Y$ consists of just one point, then $G$ is a Lie group, $V=T$ and $\omega$ is the left-invariant Maurer-Cartan form of $G$. The Lie algebra $\mathfrak{g}$ of $G$ endowed with the discrete topology is a transitive Lie algebra. Applying the above results to $\omega$, we see that $J_{1}(T ; \omega)$ is a formally transitive and formally integrable Lie equation on $X=G$, and that for $g \in G$ the image of the monomorphism of transitive Lie algebras

$$
\iota_{g}: \mathfrak{g} \rightarrow J_{\infty}(T)_{g}
$$

sending $\xi \in \mathfrak{g}$ into $j_{\infty}(\iota(\xi))(g)$, where $\iota(\xi)$ is the right-invariant vector field on $G$ whose value at the identity element of $G$ is $\xi$, is the transitive subalgebra $J_{\infty}(T ; \omega)_{g}$ of $J_{\infty}(T)_{g}$. The solutions of $J_{1}(T ; \omega)$ (resp. $\left.Q_{1}(T ; \omega)\right)$ over connected subsets of $X$ are the restrictions of right-invariant vector fields on $G$ (resp. right-translations of $G$ ). 
We no longer suppose that $Y$ is a point, and assume now that $G$ is the trivial bundle of Lie groups $\mathrm{pr}_{1}: Y \times G_{0} \rightarrow Y$, where $G_{0}$ is a Lie group. If $\mathfrak{g}_{0}$ is the Lie algebra of $G_{0}$ endowed with the discrete topology, then $\mathrm{g}$ is the trivial vector bundle $Y \times g_{0}$ of Lie algebras, and for $y \in Y$ we have the isomorphism of linearly compact Lie algebras

$$
\mathrm{g}_{0} \otimes F_{y} \rightarrow J_{\infty}(\mathrm{g} ; Y)_{y}
$$

sending $\xi \otimes j_{\infty}(f)(y)$ into $j_{\infty}(s)(y)$, where $f$ is a real-valued function on $Y$ and $s$ is the section of $g$ over $Y$ defined by $s(b)=f(b) \xi$, for $b \in Y$. Moreover, the left-invariant Maurer-Cartan form $\omega_{0}: T_{G_{0}} \rightarrow \mathrm{g}_{0}$ of the Lie group $G_{0}$ determines a section of $V^{*} \otimes_{X} \mathrm{~g}$ over $X$, which is in fact equal to the MaurerCartan form $\omega$ of the bundle $G$ of Lie groups. According to the discussion at the end of $\$ 22$, the sequence (22.36) is exact for $x \in X$ and $1 \leqslant k \leqslant \infty$; therefore $J_{1}(T ; \omega)$ is a formally transitive and formally integrable $\rho$-projectable Lie equation whose $k$ th prolongation is $J_{k+1}(T ; \omega)$. From (22.32), we deduce that

$$
\left[\tilde{J}_{2}(\widetilde{T} ; \omega), J_{1}(\mathscr{V} ; \omega)\right] \subset J_{1}(\mathscr{V} ; \omega)
$$

if $\tilde{J}_{2}(T ; \omega)=\nu^{-1} J_{2}(T ; \omega)$, and that $J_{\infty}(V ; \omega)_{x}$ is a closed ideal of the transitive Lie algebra $J_{\infty}(T ; \omega)_{x}$, for $x \in X$. With respect to a structure of analytic manifold on $Y$ compatible with the structure of differentiable manifold of $Y$ and the structure of analytic Lie group of $G_{0}$, the Lie equations $J_{1}(V ; \omega)$ and $J_{1}(T ; \omega)$ are analytic and satisfy (24.19). Hence for $x \in X$ and $j>0$, we have

$$
\begin{aligned}
& H^{j}\left(J_{\infty}(T ; \omega)_{x}, J_{\infty}(V ; \omega)_{x}\right)=H^{j}\left(J_{1}(V ; \omega)\right)_{x}=0 \\
& \tilde{H}^{1}\left(J_{\infty}(T ; \omega)_{x}, J_{\infty}(V ; \omega)_{x}\right)=\tilde{H}^{1}\left(J_{1}(V ; \omega)\right)_{x}=0
\end{aligned}
$$

by Proposition 22.4 and Theorem 24.1; moreover by [16, Corollary 13.1] and Corollary 10.1, we obtain

$$
\begin{aligned}
H^{j}\left(J_{\infty}(T ; \omega)_{x}\right) & =H^{j}\left(J_{1}(T ; \omega)\right)_{x}=0, \\
\tilde{H}^{1}\left(J_{\infty}(T ; \omega)_{x}\right) & =\tilde{H}^{1}\left(J_{1}(T ; \omega)\right)_{x}=0,
\end{aligned}
$$

for all $x \in X$ and $j>0$. According to Proposition 22.4, the characteristic variety $\mathcal{V}_{x}\left(J_{1}(V ; \omega), \mathbf{C}\right)$ of $J_{1}(V ; \omega)$ is equal to $\rho_{x}^{*} T_{Y, \rho(x)} \otimes \mathbf{C}$. Hence by Theorem 16.4 (i), the characteristic variety $\mathcal{V}\left(J_{\infty}(T ; \omega)_{x}, J_{\infty}(V ; \omega)_{x}, \mathbf{C}\right)$ is equal to the image of $\rho_{x}^{*} T_{Y, \rho(x)} \otimes \mathrm{C}$ under the injective mapping

$$
\pi_{0}^{*} \circ \nu^{*-1} \otimes \mathrm{id}: T_{x}^{*} \otimes \mathbf{C} \rightarrow J_{\infty}(T ; \omega)_{x}^{*} \otimes \mathbf{C} .
$$


If $x=(y, g) \in Y \times G_{0}$, setting $Z=G_{0}, z=g$ and $\omega_{Z}=\omega_{0}$, according to the discussion at the end of $\S 22$, we see that under the isomorphism (21.4) the image of the semi-direct product

$$
M=\left(J_{\infty}\left(T_{G_{0}} ; \omega_{0}\right)_{g} \otimes F_{y}\right) \oplus J_{\infty}\left(T_{Y} ; Y\right)_{y}
$$

is the transitive subalgebra $J_{\infty}(T ; \omega)_{x}$ of $J_{\infty}(T ; \rho)_{x}$ and that the image of the closed ideal $J_{\infty}\left(T_{G_{0}} ; \omega_{0}\right)_{g} \otimes F_{y}$ of $M$ is the closed ideal $J_{\infty}(V ; \omega)_{x}$ of $J_{\infty}(T ; \omega)_{x}$. In fact, the diagram

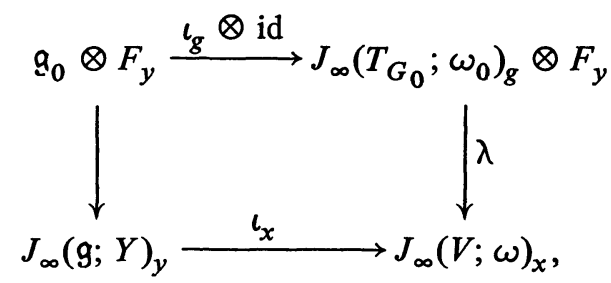

whose vertical arrows are the isomorphism (24.18) and the restriction of the isomorphism (21.3) and where $\iota_{g}: g_{0} \rightarrow J_{\infty}\left(T_{G_{0}} ; \omega_{0}\right)$ and $\iota_{x}$ are isomorphisms, is commutative. If $g_{0}$ is a non-abelian and simple Lie algebra, then $J_{\infty}\left(T_{G_{0}} ; \omega_{0}\right)_{g} \otimes F_{y}$ and $J_{\infty}(V ; \omega)_{x}$ are non-abelian minimal closed ideals of $M$ and $J_{\infty}(T ; \omega)_{x}$ respectively; moreover

$$
\begin{gathered}
M \supset J_{\infty}\left(T_{G_{0}} ; G_{0}\right)_{g} \otimes F_{y} \supset 0, \\
J_{\infty}(T ; \omega)_{x} \supset J_{\infty}(V ; \omega)_{x} \supset 0
\end{gathered}
$$

are Jordan-Hölder sequences for the transitive Lie algebras $M$ and $J_{\infty}(T ; \omega)_{x}$ respectively. From the commutativity of diagram (21.6), we deduce that the characteristic variety $\mathscr{V}\left(M, J_{\infty}\left(T_{G_{0}} ; \omega_{0}\right)_{g} \otimes F_{y}, \mathrm{C}\right)$ of the closed ideal $J_{\infty}\left(T_{G_{0}} ; \omega_{0}\right)_{g} \otimes F_{y}$ of $M$ over $\mathbf{C}$ is equal to the image of $T_{Y, y}^{*} \otimes \mathbf{C}$ under the injective mapping (21.7), with $L=J_{\infty}\left(T_{G_{0}} ; \omega_{0}\right)_{g}$.

From the above discussion, we obtain:

Proposition 24.5. Let $G_{0}$ be a Lie group and $\mathrm{g}_{0}$ the Lie algebra of $G_{0}$ endowed with the discrete topology. If $\omega_{0}$ is the Maurer-Cartan form of $G_{0}$, then for $g \in G$ we have an isomorphism

$$
\iota_{g}: g_{0} \rightarrow J_{\infty}\left(T_{G_{0}} ; \omega_{0}\right)_{g} .
$$

If $\omega$ is the Maurer-Cartan form of the bundle of Lie groups $X=Y \times G_{0}$, for $x \in X$ with $\rho(x)=y$, there is an isomorphism of transitive Lie algebras

$$
\left(g_{0} \otimes F_{y}\right) \oplus J_{\infty}\left(T_{Y} ; Y\right)_{y} \rightarrow J_{\infty}(T ; \omega)_{x}
$$

mapping the closed ideal $\mathrm{g}_{0} \otimes F_{y}$ of the semi-direct product onto the closed ideal $J_{\infty}(V ; \omega)_{x}$ of $J_{\infty}(T ; \omega)_{x}$. 
Let $g$ be a finite-dimensional real Lie algebra; then $g$ endowed with the discrete topology is a transitive Lie algebra. Let $G_{0}$ be a Lie group whose Lie algebra $g_{0}$ is isomorphic to $\mathrm{g}$. We deduce from (24.20) and Proposition 24.5 the following:

Theorem 24.2. Let $\mathrm{g}$ be a finite-dimensional real Lie algebra. Then for $y \in Y$, we have

$$
H^{j}\left(\left(\mathfrak{g} \otimes F_{y}\right) \oplus J_{\infty}\left(T_{Y} ; Y\right)_{y}, \mathfrak{g} \otimes F_{y}\right)=0
$$

for $j>0$, and

$$
\tilde{H}^{1}\left(\left(\mathfrak{g} \otimes F_{y}\right) \oplus J_{\infty}\left(T_{Y} ; Y\right)_{y}, \mathfrak{g} \otimes F_{y}\right)=0
$$

If we take $Y$ to be a point in Theorem 24.2, we obtain:

Corollary 24.1. Let $\mathrm{g}$ be a finite-dimensional real Lie algebra. Then for $j>0$, we have

$$
H^{j}(\mathfrak{g})=0, \quad \tilde{H}^{1}(\mathfrak{g})=0
$$

\section{The contact algebra with parameters}

Assume throughout this section that the rank $q$ of $V$ is $>1$. Let $\omega$ be a nowhere vanishing section of $V$ and let $W$ be the sub-bundle of $V$ of codimension one consisting of all vectors $\xi \in V$ satisfying $\langle\xi, \omega\rangle=0$. We say that $\omega$ is a contact form along the fibers of $\rho$ if the mapping

$$
W \rightarrow W^{*}
$$

sending $\xi$ into $\left(\xi \pi d_{X / Y} \omega\right)_{\mid W}$ is an isomorphism of vector bundles. This condition is equivalent to the fact that the rank of $V$ is odd and equal to $q=2 p+1$, with $p \geqslant 1$, and that the section $\omega \wedge\left(d_{X / Y} \omega\right)^{p}$ of $\wedge{ }^{q} V^{*}$ does not vanish at any point of $X$. We assume throughout this section that $\omega$ is a contact form along the fibers of $\rho$. The restriction $\omega_{X_{y}}$ of $\omega$ to a fiber $X_{y}$, with $y \in Y$, is a contact form on the odd-dimensional manifold $X_{y}$. If $X$ is the product $Y \times Z$ of $Y$ with a manifold $Z$ and $\rho: X \rightarrow Y$ is the projection onto the first factor, and if $\omega_{Z}$ is a contact form on $Z$, the section $\tilde{\omega}_{Z}$ of $V^{*}$ determined by $\omega_{Z}$ is a contact form along the fibers of $\rho$.

Let $(z, y)$ be a local coordinate for $X$ on an open neighborhood of $a \in X$ compatible with $\rho$, where $y=\left(y^{1}, \cdots, y^{m}\right)$ is a local coordinate for $Y$ on a neighborhood of $\rho(a)$. According to Darboux's theorem with parameters, there are functions $t, v^{1}, \cdots, v^{p}, w^{1}, \cdots, w^{p}$ on a neighborhood of $a$ such that $\omega$ is the restriction to $V$ of the 1 -form

$$
d t+\sum_{j=1}^{p}\left(w^{j} d v^{j}-v^{j} d w^{j}\right)
$$


and $t(a)=v^{j}(a)=w^{j}(a)=0$, for $1 \leqslant j \leqslant p$. From the properties of $\omega$, we see that

$$
\left(t, v^{1}, \cdots, v^{p}, w^{1}, \cdots, w^{p}, y^{1}, \cdots, y^{m}\right)
$$

is a local coordinate on a neighborhood of $a \in X$ compatible with $\rho$.

Proposition 25.1. Let $\omega^{\prime}, \omega^{\prime}$ be two contact forms along the fibers of $\rho$. If $a, b \in X$, there is a $\rho$-projectable local diffeomorphism $f: X \rightarrow X$ defined on $a$ neighborhood of a such that $f(a)=b$ and $f^{*} \omega=\omega^{\prime}$; moreover if $\rho(a)=\rho(b)$, there is such a diffeomorphism inducing the identity mapping of $Y$.

Proof. There are local coordinates $(z, y)$ and $\left(z^{\prime}, y^{\prime}\right)$ on neighborhoods $U$ of $a$ and $U^{\prime}$ of $b$ respectively compatible with $\rho$, where $y=\left(y^{1}, \cdots, y^{m}\right)$, $y^{\prime}=\left(y^{\prime 1}, \cdots, y^{\prime m}\right)$ are local coordinates for $Y$ on $\rho(U)$ and $\rho\left(U^{\prime}\right)$ respectively. If $\rho(a)=\rho(b)$, we may assume that $y=y^{\prime}$. From the above discussion, we may also suppose that $\omega$ is the restriction to $V$ of the form (25.2) on $U$, with $t=z^{1}, v^{j}=z^{j+1}$ and $w^{j}=z^{j+p+1}$, for $1 \leqslant j \leqslant p$, that $\omega^{\prime}$ is the restriction to $V$ of the form (25.2) on $U^{\prime}$, with $t=z^{\prime 1}, v^{j}=z^{j+1}$ and $w^{j}=z^{j+p+1}$, for $1 \leqslant j \leqslant p$, and that $z(a)=z^{\prime}(b)$. Let $\bar{f}$ be a local diffeomorphism $Y \rightarrow Y$ defined on a neighborhood of $\rho(a)$ with $\bar{f}(\rho(a))=\rho(b)$; if $\rho(a)=\rho(b)$, we suppose that $\bar{f}$ is the identity mapping of $Y$. The mapping $f$ defined on a neighborhood of $a$ sending the point $x$ of $U$ with coordinate $(z, y)$ into the point of $U^{\prime}$ whose $\left(z^{\prime}, y^{\prime}\right)$ coordinate is equal to $\left(z, y^{\prime}(\bar{f}(\rho(x)))\right)$ is a $\rho$-projectable diffeomorphism and satisfies $f(a)=b$ and $f^{*} \omega^{\prime}=\omega$, since $z^{\prime} \circ f=z$.

If $\{\omega\}$ is the sub-bundle of $V$ generated by the section $\omega$ and $\varphi: V^{*} \rightarrow W^{*}$ is the canonical projection, we have the exact sequence

$$
0 \rightarrow\{\omega\} \rightarrow V^{*} \stackrel{\varphi}{\rightarrow} W^{*} \rightarrow 0 .
$$

Let $P: \mathcal{V} \rightarrow \mathcal{Q}^{*}$ be the first-order linear differential operator sending $\xi$ into $\varphi \mathscr{L}(\xi) \omega$. We denote by $E$ the vector bundle over $X$ which is the quotient of $V^{*} \oplus \wedge{ }^{2} W^{*}$ by its sub-bundle generated by the section $\left(\omega,\left(d_{X / Y} \omega\right)_{\mid \wedge}{ }^{2} W\right)$, and we let $\psi: V^{*} \oplus \wedge^{2} W^{*} \rightarrow E$ be the canonical projection. Let $Q: \mathcal{\sigma}_{\rho} \rightarrow \mathcal{E}$ be the differential operator sending $\xi$ into $\psi\left(\mathcal{L}(\xi) \omega,\left(\mathcal{L}(\xi) d_{X / Y} \omega\right)_{\mid \wedge}{ }^{2} W\right)$, and

$$
p_{k-1}(Q): J_{k}(T ; \rho) \rightarrow J_{k-1}(E)
$$

be the morphism of vector bundles sending $j_{k}(\xi)(x)$ into $j_{k-1}(Q \xi)(x)$, where $\xi \in \widetilde{J}_{\rho, x}$.

Let $f: X \rightarrow X$ be a $\rho$-projectable local diffeomorphism defined on a neighborhood $U$ of $a \in X$, with $f(a)=b$, and $\xi$ a $\rho$-projectable vector field on $U$; then $f_{*} \xi$ is a $\rho$-projectable vector field on $f(U)$. If $\phi=j_{k+1}(f)(a)$, the isomorphism

$$
\phi: J_{k}(T ; \rho)_{a} \rightarrow J_{k}(T ; \rho)_{b}
$$


sends $j_{k}(\xi)(a)$ into $j_{k}\left(f_{*} \xi\right)(b)$ and $J_{k}(V)_{a}$ onto $J_{k}(V)_{b}$. Let

$$
\phi: J_{k-1}\left(\wedge V^{*}\right)_{b} \rightarrow J_{k-1}\left(\wedge V^{*}\right)_{a}
$$

be the isomorphism sending $j_{k-1}(\alpha)(b)$ into $j_{k-1}\left(f^{*} \alpha\right)(a)$, where $\alpha \in \wedge \mathcal{V}_{b}^{*}$. If $f^{*} \omega=\omega$, since $f^{*} d_{X / Y} \omega=d_{X / Y} \omega$, by passage to the quotient the mapping (25.3) determines isomorphisms

$$
\begin{gathered}
\phi: J_{k-1}\left(W^{*}\right)_{b} \rightarrow J_{k-1}\left(W^{*}\right)_{a}, \\
\phi: J_{k-1}(E)_{b} \rightarrow J_{k-1}(E)_{a} .
\end{gathered}
$$

From relation (22.16), it follows that the diagrams

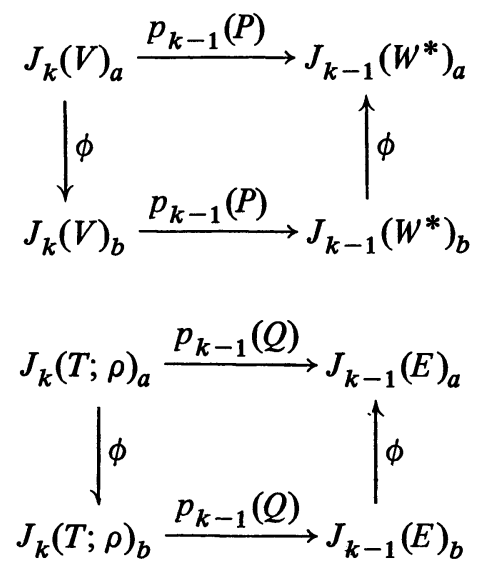

are commutative. From Proposition 25.1, with $\omega^{\prime}=\omega$, we deduce that $p_{k-1}(P)$, $p_{k-1}(Q)$ and the restriction of $p_{k-1}(Q)$ to $J_{k}(V)$ are morphisms of vector bundles of constant rank.

Let $J_{k}(V ; W), R_{k}(V ; \omega)$ and $R_{k}(\omega)=R_{k}(T ; \omega)$ be the kernels of the morphisms

$$
\begin{gathered}
p_{k-1}(P): J_{k}(V) \rightarrow J_{k-1}\left(W^{*}\right), \\
p_{k-1}(Q): J_{k}(V) \rightarrow J_{k-1}(E), \\
p_{k-1}(Q): J_{k}(T ; \rho) \rightarrow J_{k-1}(E),
\end{gathered}
$$

respectively. From the above discussion, we see that $J_{k}(V ; W), R_{k}(V ; W)$ and $R_{k}(\omega)$ are vector bundles and that $\pi_{k}: J_{k+l}(V ; W) \rightarrow J_{k}(V ; W)$ is of constant rank for all $k, l \geqslant 1$; moreover the $k$ th prolongations of $J_{1}(V ; W), R_{1}(V ; \omega)$ 
and $R_{1}(\omega)$ are $J_{k+1}(V ; W), R_{k+1}(V ; \omega)$ and $R_{k+1}(\omega)$ respectively. The subbundle $J_{k}(V ; W)$ of $J_{k}(V)$ depends only on $V$ and $W$, and is equal to

$$
\left\{\xi \in J_{k}(V) \mid\left[\xi, J_{k}(W)\right] \subset J_{k-1}(W)\right\},
$$

or to

$$
\left\{\xi \in J_{k}(V) \mid \mathcal{L}(\tilde{\xi}) J_{k}(\mathscr{W}) \subset J_{k}(W), \text { with } \tilde{\xi}=\nu^{-1} \xi\right\} .
$$

Indeed, if $\xi=j_{k}(\zeta)(x)$, with $\zeta \in \mathscr{V}_{x}, x \in X$, then $\xi$ belongs to the kernel of $p_{k-1}(P)$ if and only if

$$
j_{k-1}(\langle\eta, \mathcal{L}(\zeta) \omega\rangle)(x)=-j_{k-1}(\langle[\zeta, \eta], \omega\rangle)(x)=0,
$$

for all $\eta \in \mho_{x}$, or equivalently if and only if $j_{k-1}([\zeta, \eta])(x)$ is an element of $J_{k-1}(W)$ for all $\eta \in \mathcal{Q} \int_{x}$. Since

$$
\left[\xi, j_{k}(\eta)(x)\right]=\mathcal{L}(\tilde{\xi}) j_{k-1}(\eta)=j_{k-1}([\zeta, \eta])(x),
$$

where $\tilde{\xi}=\nu^{-1} \xi, \eta \in \mathcal{W}$, we obtain the above descriptions of the kernel of $p_{k-1}(P)$. Moreover $j_{k}(\zeta)(x)$, with $\zeta \in \mathcal{V}_{x}$, belongs to $J_{k}(V ; W)$ if and only if there is an element $f \in \mathcal{O}_{X, x}$ such that

$$
j_{k-1}(\mathcal{L}(\zeta) \omega-f \omega)(x)=0
$$

We denote by $\bar{\omega}$ the section $\nu^{*-1} \omega$ of $J_{0}(V)^{*}$. An element $\xi \in J_{1}(V)$ belongs to $J_{1}(V ; W)$ if and only if $\mathcal{L}(\tilde{\xi}) \bar{\omega}=c \bar{\omega}$ for some $c \in \mathbf{R}$, where $\tilde{\xi}=\nu^{-1} \xi$, or equivalently if and only if $(\mathcal{L}(\tilde{\xi}) \bar{\omega}) \wedge \bar{\omega}=0$. It follows that $J_{1}(V ; W)$ is a Lie equation whose solutions are the vertical vector fields $\xi$ satisfying $\mathcal{L}(\xi) \omega=f \omega$, for some real-valued function $f$ on $X$. If $\xi$ is a solution of $J_{1}(V ; W)$ over an open set $U$ and $g$ is a real-valued function on $\rho(U)$, then $(g \circ \rho) \xi$ is a solution of $J_{1}(V ; W)$ over $U$. By (22.18), the restriction of $P$ to $\mho$ is the mapping ข $\rightarrow$ ข ${ }^{*}$ induced by the isomorphism of vector bundles (25.1). Therefore the sequences

$$
\begin{aligned}
0 \rightarrow J_{k}(V ; W) \rightarrow & J_{k}(V) \stackrel{p_{k-1}(P)}{\longrightarrow} J_{k-1}\left(W^{*}\right) \rightarrow 0, \\
& \mathcal{V}^{P} \rightarrow \mathscr{P}^{*} \rightarrow 0
\end{aligned}
$$

are exact. From [10, Theorem 3], we infer that

$$
H^{j}\left(J_{1}(V ; W)\right)=0, \quad \text { for } j>0 .
$$

From the definition of the operator $Q$, we see that $j_{k}(\zeta)(x)$, with $\zeta \in \mathcal{V}_{x}$ (resp. $\zeta \in \mathcal{T}_{\rho, x}$ ), belongs to $R_{k}(V ; \omega)$ (resp. $R_{k}(\omega)$ ) if and only if there are elements $f \in \mathcal{O}_{X, x}$ and $\alpha \in \mathcal{V}_{x}^{*}$ such that

$$
\begin{gathered}
j_{k-1}(\mathcal{L}(\zeta) \omega-f \omega)(x)=0, \\
j_{k-1}\left(\mathcal{L}(\zeta) d_{X / Y} \omega-f d_{X / Y} \omega-\alpha \wedge \omega\right)(x)=0 .
\end{gathered}
$$


Therefore

$$
\begin{gathered}
{\left[R_{k+1}(\omega), R_{k+1}(V ; \omega)\right] \subset R_{k}(V ; \omega),} \\
{\left[R_{k+1}(\omega), R_{k+1}(\omega)\right] \subset R_{k}(\omega),} \\
R_{k}(V ; \omega) \subset J_{k}(V ; W) .
\end{gathered}
$$

We denote by $\bar{\Omega}$ the section $\nu^{*-1} d_{X / Y} \omega$ of $\wedge^{2} J_{0}(V)^{*}$. An element $\xi \in J_{1}(V)$ (resp. $J_{1}(T ; \rho)$ ) belongs to $R_{1}(V ; \omega)$ (resp. $R_{1}(\omega)$ ) if and only if there are elements $c \in \mathbf{R}$ and $\alpha \in J_{0}(V)^{*}$ such that

$$
\mathcal{L}(\tilde{\xi}) \bar{\omega}=c \bar{\omega}, \quad \mathcal{L}(\tilde{\xi}) \bar{\Omega}=c \bar{\Omega}+\alpha \wedge \bar{\omega} .
$$

If $j_{k+1}(\zeta)(x)$, with $\zeta \in \mathscr{V}_{x}$, belongs to $J_{k+1}(V ; W)$ and $f$ is an element of $\vartheta_{X, x}$ such that $j_{k}(\ell(\zeta) \omega-f \omega)(x)=0$, then

$$
j_{k-1}\left(d_{X / Y}(\varrho(\zeta) \omega-f \omega)\right)(x)=0,
$$

or equivalently

$$
j_{k-1}\left(\varrho(\zeta) d_{X / Y} \omega-f d_{X / Y} \omega-d_{X / Y} f \wedge \omega\right)(x)=0 ;
$$

thus $j_{k}(\zeta)(x) \in R_{k}(V ; \omega)$ and so

$$
\pi_{k} J_{k+1}(V ; W) \subset R_{k}(V ; \omega) .
$$

From (25.8) and (25.10), it follows that

$$
\begin{aligned}
R_{\infty}(V ; \omega) & =J_{\infty}(V ; W), \\
\operatorname{Sol}\left(R_{1}(V ; \omega)\right) & =\operatorname{Sol}\left(J_{1}(V ; W)\right) .
\end{aligned}
$$

The morphism of vector bundles

$$
\psi_{k-1}: J_{k}(V ; W) \rightarrow J_{k-1}\left(\wedge^{2} W^{*}\right),
$$

sending $j_{k}(\zeta)(x)$, with $\zeta \in \mathscr{V}_{x}$, into

$$
j_{k-1}\left(\left(\varrho(\zeta) d_{X / Y} \omega-f d_{X / Y} \omega\right)_{\mid \wedge{ }^{2} W}\right)(x),
$$

where $f$ is an element of $\vartheta_{X, x}$ satisfying

$$
j_{k-1}(\mathcal{L}(\zeta) \omega-f \omega)(x)=0,
$$

is well-defined, and by (25.5) its kernel is equal to $R_{k}(V ; \omega)$. We set $R_{0}(V ; \omega)$ $=J_{0}(V)$; if

$$
g_{k} \subset S^{k} J_{0}(T)^{*} \otimes J_{0}(V), \quad h_{k} \subset S^{k} J_{0}(T)^{*} \otimes J_{0}(V)
$$

are the kernels of the mappings

$$
\begin{aligned}
\pi_{k-1}: R_{k}(V ; \omega) & \rightarrow J_{k-1}(V), \\
\pi_{k-1}: \pi_{k} R_{\infty}(V ; \omega) & \rightarrow J_{k-1}(V)
\end{aligned}
$$


respectively, where $J_{-1}(V)=0$, we have

$$
h_{k} \subset g_{k},
$$

for $k \geqslant 0$, and $g_{0}=J_{0}(V)$.

If $U$ is a finite-dimensional vector space, for $u \in U^{*} \otimes U, \alpha \in \wedge U^{*}$, we obtain an element $u \pi \alpha$ of $\wedge U^{*}$. If $\alpha \in U^{*}$, then

$$
\langle\xi, u \pi \alpha\rangle=\langle u(\xi), \alpha\rangle
$$

for $\xi \in U$; if $\alpha \in \wedge^{2} U^{*}$, then

$$
\langle\xi \wedge \eta, u \pi \alpha\rangle=\langle u(\xi) \wedge \eta, \alpha\rangle+\langle\xi \wedge u(\eta), \alpha\rangle,
$$

for $\xi, \eta \in U$. If $u \in J_{0}(T)^{*} \otimes J_{0}(V), \alpha \in \wedge J_{0}(V)^{*}$, we also write $u \pi \alpha$ for the element $v \pi \alpha$ of $\wedge J_{0}(V)^{*}$, where $v=\theta(u)$ is the restriction of $u$ to $J_{0}(V)$. From the description (25.9) of $R_{1}(V ; \omega)$, we see that $u \in J_{0}(T)^{*} \otimes J_{0}(V)$ belongs to $g_{1}$ if and only if there are elements $c \in \mathbf{R}$ and $\alpha \in J_{0}(V)^{*}$ such that

$$
u \pi \bar{\omega}=c \bar{\omega}, \quad u \pi \bar{\Omega}=c \bar{\Omega}+\alpha \wedge \bar{\omega} .
$$

The sub-bundle $\rho\left(J_{0}\left(T_{Y}\right)^{*}\right)$ of $J_{0}(T)^{*}$, whose fiber over $x \in X$ is $\nu^{*-1}\left(\rho^{*} T_{Y, \rho(x)}\right)$, is equal to the annihilator of $J_{0}(V)$; hence $\rho\left(J_{0}\left(T_{Y}\right)^{*}\right) \otimes J_{0}(V)$ is a sub-bundle of $g_{1}$ and the sequence

$$
0 \rightarrow \rho\left(J_{0}\left(T_{Y}\right)^{*}\right) \otimes J_{0}(V) \rightarrow g_{1} \stackrel{\theta}{\rightarrow} J_{0}(V)^{*} \otimes J_{0}(V)
$$

is exact. The image $\bar{g}_{1}=\theta\left(g_{1}\right)$ of $g_{1}$ consists of all $u \in J_{0}(V)^{*} \otimes J_{0}(V)$ satisfying (25.15) for some $c \in \mathbf{R}$ and $\alpha \in J_{0}(V)^{*}$. If $\{\bar{\omega}\}$ is the sub-bundle of $J_{0}(V)^{*}$ generated by the section $\bar{\omega}$ and $\Omega$ denotes the restriction of $\bar{\Omega}$ to $\wedge^{2} J_{0}(W)$, the kernel of the mapping

$$
\bar{g}_{1} \rightarrow\{\bar{\omega}\}
$$

sending $u \in \bar{g}_{1}$ into $u \pi \bar{\omega}$, consists of all $u \in J_{0}(V)^{*} \otimes J_{0}(W)$ satisfying $v \pi \Omega$ $=0$, where $v$ is the restriction of $u$ to $J_{0}(W)$, and contains the subbundle $\{\bar{\omega}\} \otimes J_{0}(W)$ of $J_{0}(V)^{*} \otimes J_{0}(V)$. Indeed, if $u \in J_{0}(V)^{*} \otimes J_{0}(V)$, by (25.13) we see that $u \pi \bar{\omega}=0$ if and only if $u$ belongs to $J_{0}(V)^{*} \otimes J_{0}(W)$; for $u \in J_{0}(V)^{*} \otimes J_{0}(W)$, by (25.14) the restriction of $u \pi \bar{\Omega}$ to $\wedge^{2} J_{0}(W)$ is equal to $v \pi \Omega$. Let $a \in X$ and choose an element $\xi^{0}$ of $J_{0}(V)_{a}$ not belonging to $J_{0}(W)_{a}$, i.e., satisfying $\left\langle\xi^{0}, \bar{\omega}\right\rangle \neq 0$. The unique element $u_{0}$ of $\left(J_{0}(V)^{*} \otimes J_{0}(V)\right)_{a}$, whose restriction to $J_{0}(W)_{a}$ is the identity mapping of $J_{0}(W)_{a}$ and which verifies $u_{0}\left(\xi^{0}\right)=2 \xi^{0}$, belongs to $\bar{g}_{1, a}$ and satisfies $u_{0} \pi \bar{\omega}=2 \bar{\omega}$, according to (25.13) and (25.14); therefore the mapping (25.17) is surjective. If $\mathfrak{h}$ is the subspace of $\bar{g}_{1, a}$ consisting of the elements $u$ of $\left(J_{0}(V)^{*} \otimes J_{0}(W)\right)_{a}$ satisfying $u\left(\xi^{0}\right)=0$ 
and $v \pi \Omega=0$, where $v$ is the restriction of $u$ to $J_{0}(W)_{a}$, then we easily see that the fiber of the kernel of (25.17) over $a$ is equal to

$$
\mathfrak{h} \oplus\left(\{\bar{\omega}\} \otimes J_{0}(W)\right)_{a} .
$$

Hence if $\left\{u_{0}\right\}$ is the subspace of $\bar{g}_{1, a}$ generated by $u_{0}$, we obtain the decomposition

$$
\bar{g}_{1, a}=\mathfrak{h} \oplus\left(\{\bar{\omega}\} \otimes J_{0}(W)\right)_{a} \oplus\left\{u_{0}\right\},
$$

which depends only on the choice of the element $\xi^{0}$ of $J_{0}(V)_{a}$ satisfying $\left\langle\xi^{0}, \bar{\omega}\right\rangle \neq 0$ (see [37, §1.14]).

The proof of the following proposition is based in part on [23, §7].

Proposition 25.2. If $\omega$ is a contact form along the fibers of $\rho$, then $R_{1}(V ; \omega)$ is a formally integrable and integrable Lie equation, with

$$
\begin{aligned}
R_{k}(V ; \omega) & =\pi_{k} J_{k+1}(V ; W), \\
\pi_{0} R_{1}(V ; \omega) & =J_{0}(V) .
\end{aligned}
$$

Moreover $g_{1}$ is involutive and

$$
H^{j}\left(R_{k}(V ; \omega)\right)=0, \text { for } j>0 .
$$

For $a \in X$, the mapping

$$
\operatorname{Sol}\left(R_{1}(V ; \omega)\right)_{a} \rightarrow \vartheta_{X, a},
$$

sending $\xi$ into $\xi \pi \omega$ is an isomorphism, and the characteristic variety of $R_{1}(V ; \omega)$ over $\mathbf{C}$ is equal to $T_{a}^{*} \otimes \mathbf{C}$.

Proof. Let $a \in X$. If $\xi$ is a vertical vector field on a neighborhood of $a$, we write $\sigma_{0}(\xi)=\nu \xi(a)$ and, whenever $j_{k-1}(\xi)(a)=0$, with $k \geqslant 1$, we denote by $\sigma_{k}(\xi)$ the element $j_{k}(\xi)(a)$ of $\left(S^{k} J_{0}(T)^{*} \otimes J_{0}(V)\right)_{a}$, which belongs to $h_{k, a}$ if $\xi$ is a solution of $R_{1}(V ; \omega)$. Let $(t, v, w, y)$ be a local coordinate on a neighborhood $U$ of $a$ compatible with $\rho$, where $v=\left(v^{1}, \cdots, v^{p}\right), w=\left(w^{1}, \cdots, w^{p}\right)$ and $y=\left(y^{1}, \cdots, y^{m}\right)$ is a local coordinate for $Y$ on $\rho(U)$. We write $z=\left(z^{1}, \cdots, z^{q}\right)$ for $(t, v, w)$. Assume that $\omega$ is the restriction to $V$ of the form (25.2) on $U$, and that

$$
t(a)=0, \quad v(a)=w(a)=0, \quad y(\rho(a))=0 ;
$$

then $d_{X / Y} \omega$ is the restriction to $\wedge^{2} V$ of the 2-form

$$
2 \sum_{j=1}^{p} d w^{j} \wedge d v^{j}
$$

on $U$. 
Since $\omega(a)=(d t)_{a}$, the subspace $W_{a}$ of $V_{a}$ is spanned by the elements $\left(\partial / \partial v^{j}\right)_{a},\left(\partial / \partial w^{j}\right)_{a}$, with $1 \leqslant j \leqslant p$. We may therefore identify the quotient $W_{a}^{*}$ of $T_{a}^{*}$ with the subspace

$$
\left\{\xi \in T_{a}^{*} \mid\left\langle\frac{\partial}{\partial t}, \xi\right\rangle=\left\langle\frac{\partial}{\partial y^{i}}, \xi\right\rangle=0, \text { for } i=1, \cdots, m\right\}
$$

of $T_{a}^{*}$, and hence also the quotients $S^{k} W_{a}^{*}$ of $S^{k} T_{a}^{*}$ and $S^{k} J_{0}(W)_{a}^{*}$ of $S^{k} J_{0}(T)_{a}^{*}$ with subspaces of $S^{k} T_{a}^{*}$ and $S^{k} J_{0}(T)_{a}^{*}$ respectively. In fact, $\varepsilon\left(S^{k} W_{a}^{*}\right)$ is equal to the space of $k$-jets

$$
\left\{\begin{array}{l|l}
j_{k}(f)(a) & \begin{array}{l}
f=f(v, w) \text { is a homogeneous } \\
\text { polynomial of degree } k \text { in }(v, w)
\end{array}
\end{array}\right\} .
$$

If $\eta \in J_{k+1}(T)$, let

$$
\bar{\delta}_{\pi_{0} \eta}=\bar{\delta}_{\eta}: S^{k+1} J_{0}(T) * \otimes J_{0}(V) \rightarrow S^{k} J_{0}(T)^{*} \otimes J_{0}(V)
$$

be the mapping sending $u$ into

$$
\overline{\delta_{\eta}} u=\pi_{0} \eta \pi \bar{\delta} u=[\eta, u] .
$$

If $\eta=j_{k+1}(\partial / \partial t)(a)$, we write $\bar{\delta}_{t}=\bar{\delta}_{\eta}$; moreover if $\eta=j_{k+1}\left(\partial / \partial y^{i}\right)(a)$, we set $\overline{\delta_{i}}=\bar{\delta}_{\eta}$ for $1 \leqslant i \leqslant m$. Then $\left(S^{k} J_{0}(W)^{*} \otimes J_{0}(V)\right)_{a}$ is equal to the subspace

$$
\left(S^{k} J_{0}(T)^{*} \otimes J_{0}(V)\right)_{a} \cap \operatorname{ker} \bar{\delta}_{t} \cap \bigcap_{i=1}^{m} \operatorname{ker} \bar{\delta}_{i}
$$

of $\left(S^{k} J_{0}(T)^{*} \otimes J_{0}(V)\right)_{a}$.

If $\zeta=j_{k}(\xi)(a)$, where $\xi$ is a vertical vector field on a neighborhood of $a$, for any multi-index $\alpha=\left(\alpha_{1}, \cdots, \alpha_{m}\right)$ we set

$$
y^{\alpha} \cdot \zeta=j_{k+|\alpha|}\left(y^{\alpha} \cdot \xi\right)(a)
$$

If $\xi$ belongs to $\left(S^{k} J_{0}(T)^{*} \otimes J_{0}(V)\right)_{a}$, then

$$
y^{\alpha} \cdot \zeta=\sigma_{k}\left(y^{\alpha} \xi\right) \in\left(S^{k+|\alpha|} J_{0}(T)^{*} \otimes J_{0}(V)\right)_{a} .
$$

If $\zeta$ is the vertical vector field

$$
\xi=\sum_{0 \leqslant|\alpha| \leqslant k} y^{\alpha} \xi_{\alpha}
$$

with

$$
\xi_{\alpha}=\sum_{l=1}^{q} \xi_{\alpha}^{l}(z) \frac{\partial}{\partial z^{l}}, \quad 0 \leqslant|\alpha| \leqslant k,
$$

on a neighborhood of $a$, since $\omega$ is the restriction to $V$ of the form (25.2) on $U$, we see that $j_{k}(\xi)(a)$ belongs to $R_{k}(V ; \omega)_{a}$ if and only if $j_{k}\left(\xi_{\alpha}\right)(a)$ is an element 
of $R_{k-|\alpha|}(V ; \omega)_{a}$ for all $|\alpha| \leqslant k$; we infer that $\zeta=j_{k}(\xi)(a)$ belongs to $g_{k, a}$ if and only if $\zeta_{\alpha}=j_{k-|\alpha|}\left(\xi_{\alpha}\right)(a)$ is an element of $g_{k-|\alpha|, a}$ for all $|\alpha| \leqslant k$. For $k \geqslant 1$, we set

$$
g_{k}=g_{k, a} \cap\left(S^{k} J_{0}(W)^{*} \otimes J_{0}(V)\right)_{a}
$$

and $g_{0}=J_{0}(V)_{a}$. Since $g_{k+1}$ is the $k$ th prolongation of $g_{1}$, it is easily verified that

$$
\mathrm{g}_{k+1}=\left(\mathrm{g}_{1}\right)_{+k}
$$

for $k \geqslant 0$, where $\left(g_{1}\right)_{+k}$ is the $k$ th prolongation of $g_{1}$ considered as a subspace of $\left(J_{0}(T)^{*} \otimes J_{0}(V)\right)_{a}$ or of $\left(J_{0}(W)^{*} \otimes J_{0}(V)\right)_{a}$ (see [9, §6]). From the above discussion, we infer that $\zeta=j_{k}(\xi)(a)$ belongs to $g_{k, a}$ and $\bar{\delta}_{t} \zeta=0$ if and only if we may choose $\xi$ to be a vector field (25.22) with

$$
\xi_{\alpha}=\sum_{l=1}^{q} \xi_{\alpha}^{l}(v, w) \frac{\partial}{\partial z^{l}}
$$

on a neighborhood of $a$, and $j_{k-|\alpha|}\left(\xi_{\alpha}\right) \in \mathfrak{g}_{k-|\alpha|}$ for all $|\alpha| \leqslant k$. We thus have the decomposition

$$
g_{k, a} \cap \operatorname{ker} \bar{\delta}_{t}=\bigoplus_{0 \leqslant|\alpha| \leqslant k} y^{\alpha} \cdot g_{k-|\alpha|} .
$$

If $f$ is a real-valued function on $U$, the vertical vector field

$$
\begin{aligned}
\xi_{f}= & \left(\sum_{j=1}^{p}\left(v^{j} \frac{\partial f}{\partial v^{j}}+w^{j} \frac{\partial f}{\partial w^{j}}\right)-2 f\right) \frac{\partial}{\partial t} \\
& -\sum_{j=1}^{p}\left(\frac{\partial f}{\partial w^{j}}+v^{j} \frac{\partial f}{\partial t}\right) \frac{\partial}{\partial v^{j}}+\sum_{j=1}^{p}\left(\frac{\partial f}{\partial v^{j}}-w^{j} \frac{\partial f}{\partial t}\right) \frac{\partial}{\partial w^{j}}
\end{aligned}
$$

on $U$ satisfies

$$
\begin{gathered}
\left\langle\xi_{f}, \omega\right\rangle=-2 f, \\
\mathcal{L}\left(\xi_{f}\right) \omega=-2 \frac{\partial f}{\partial t} \omega ;
\end{gathered}
$$

moreover

$$
\left[\frac{\partial}{\partial t}, \xi_{f}\right]=\xi_{\frac{\partial f}{\partial t}}
$$

and

$$
(g \circ \rho) \xi_{f}=\xi_{(g \circ \rho) f},
$$

if $g$ is a real-valued function on $\rho(U)$. Thus by (25.11), $\xi_{f}$ is a solution of $R_{1}(V ; \omega)$, and the mapping (25.21) is surjective. If $\xi$ is a solution of $R_{1}(V ; \omega)$ 
over a neighborhood $U^{\prime} \subset U$ of $a$ satisfying $\xi \pi \omega=0$, then by (22.18)

$$
\mathcal{L}(\xi) \omega=\xi \pi d_{X / Y} \omega=g \omega,
$$

for some function $g$ on $U^{\prime}$. Comparing coefficients of $d t$ in the above equality, we conclude that

$$
\xi \pi d_{X / Y} \omega=0, \quad \xi \pi \omega=0 .
$$

Since $\omega$ is a contact form, we infer that $\xi=0$, and so (25.21) is an isomorphism. This argument also shows that $R_{\infty}(V ; \omega)_{a}$ consists of all jets $j_{\infty}\left(\xi_{f}\right)(a)$, with $f$ a real-valued function on $U$; therefore $h_{k, a}$ is equal to the space of all elements $\sigma_{k}(\xi)$ of $\left(S^{k} J_{0}(T)^{*} \otimes J_{0}(V)\right)_{a}$, where $\xi$ is a solution of $R_{1}(V ; \omega)$ over $U$ satisfying $j_{k-1}(\xi)(a)=0$ if $k \geqslant 1$. For $k \geqslant 0$, it follows that the space $h_{k}^{\prime}$ consisting of all elements $\sigma_{k}\left(\xi_{f}\right)=\sigma_{k}\left(\eta_{f}\right)$, where $\eta_{f}$ is the vertical vector field given by

$$
\eta_{f}=\sum_{j=1}^{p}\left(\frac{\partial f}{\partial v^{j}} \frac{\partial}{\partial w^{j}}-\frac{\partial f}{\partial w^{j}} \frac{\partial}{\partial v^{j}}\right),
$$

and $f$ is a real-valued function on $U$ satisfying $j_{k}(f)(a)=0$, and the space $h_{k}^{\prime \prime}$ consisting of all elements

$$
\sigma_{k}\left(\xi_{f}\right)=-\sigma_{k}\left(2 f \frac{\partial}{\partial t}+\frac{\partial f}{\partial t}\left(\sum_{j=1}^{p}\left(v^{j} \frac{\partial}{\partial v^{j}}+w^{j} \frac{\partial}{\partial w^{j}}\right)\right),\right.
$$

where $f=f(t, y)$ is a function on $U$ satisfying $j_{k-1}(f)(a)=0$ if $k \geqslant 1$, are subspaces of $h_{k, a}$, and that

$$
h_{k, a}=h_{k}^{\prime} \oplus h_{k}^{\prime \prime}, \quad \text { for } k \geqslant 0 .
$$

We now show by induction on $k$ that

$$
g_{k, a}=h_{k, a},
$$

for $k \geqslant 0$. First, we consider the case $k=0$. As

$$
\sigma_{0}\left(\frac{\partial}{\partial v^{j}}\right)=\sigma_{0}\left(\xi_{-w^{j}}\right), \quad \sigma_{0}\left(\frac{\partial}{\partial w^{j}}\right)=\sigma_{0}\left(\xi_{v^{j}}\right),
$$

for $1 \leqslant j \leqslant p$, and

$$
\sigma_{0}\left(\frac{\partial}{\partial t}\right)=\sigma_{0}\left(\xi_{-\frac{1}{2}}\right)
$$

we have the equality $h_{0}^{\prime}=J_{0}(W)_{a}$, and $h_{0}^{\prime \prime}$ is the subspace generated by $\nu(\partial / \partial t)_{a}$. Hence

$$
h_{0, a}=J_{0}(V)_{a}=g_{0, a} \text {, }
$$


and the mapping $\pi_{0}: R_{\infty}(V ; \omega) \rightarrow J_{0}(V)$ is surjective. Next, for $k=1$, the space $h_{1}^{\prime}$ admits a decomposition

$$
h_{1}^{\prime}=\mathfrak{a}_{1} \oplus \mathfrak{a}_{2} \oplus \mathfrak{a}_{3},
$$

where $a_{1}$ is the subspace consisting of all elements $\sigma_{1}\left(\eta_{f}\right)$, with $f=f(v, w)$ a homogeneous polynomial of degree 2 in $(v, w)$, and where $a_{2}$ (resp. $\left.a_{3}\right)$ is the subspace generated by the elements

$$
\sigma_{1}\left(t \frac{\partial}{\partial v^{j}}\right)=\sigma_{1}\left(\xi_{-t w^{j}}\right), \quad \sigma_{1}\left(t \frac{\partial}{\partial w^{j}}\right)=\sigma_{1}\left(\xi_{t v^{j}}\right),
$$

with $1 \leqslant j \leqslant p$ (resp. by the elements

$$
\sigma_{1}\left(y^{i} \frac{\partial}{\partial v^{j}}\right)=\sigma_{1}\left(\xi_{-y^{i} w^{j}}\right), \quad \sigma_{1}\left(y^{i} \frac{\partial}{\partial w^{j}}\right)=\sigma_{1}\left(\xi_{y^{i} v^{j}}\right),
$$

with $1 \leqslant i \leqslant m, 1 \leqslant j \leqslant p$ ). On the other hand, the space $h_{1}^{\prime \prime}$ is equal to the direct sum

$$
h_{1}^{\prime \prime}=\left\{\sigma_{1}\left(\xi_{-t}\right)\right\} \oplus \mathfrak{b}
$$

of the subspace $\left\{\sigma_{1}\left(\xi_{-t}\right)\right\}$ generated by $\sigma_{1}\left(\xi_{-t}\right)$ and the subspace $\mathfrak{b}$ generated by the elements

$$
\sigma_{1}\left(y^{i} \frac{\partial}{\partial t}\right)=\sigma_{1}\left(\xi_{-y^{i} / 2}\right), \text { with } 1 \leqslant i \leqslant m
$$

Clearly we have the equality

$$
\mathfrak{a}_{3} \oplus \mathfrak{b}=\left(\rho\left(J_{0}\left(T_{Y}\right)^{*}\right) \otimes J_{0}(V)\right)_{a} .
$$

Let $\xi^{0}$ be the element $\nu(\partial / \partial t)_{a}$ of $J_{0}(V)_{a}$; as $\left\langle\xi^{0}, \bar{\omega}\right\rangle=1$, we have the decomposition (25.18) of $\bar{g}_{1, a}$ corresponding to $\xi^{0}$. In fact,

$$
u_{0}=\sigma_{0}\left(2 t \frac{\partial}{\partial t}+\sum_{j=1}^{p}\left(v^{j} \frac{\partial}{\partial v^{j}}+w^{j} \frac{\partial}{\partial w^{j}}\right)\right)=\sigma_{1}\left(\xi_{-t}\right),
$$

and the mapping $\theta: g_{1, a} \rightarrow \bar{g}_{1, a}$ induces isomorphisms

$$
\begin{aligned}
& \theta: \mathfrak{a}_{1} \rightarrow \mathfrak{h}, \\
& \theta: \mathfrak{a}_{2} \rightarrow\left(\{\bar{\omega}\} \otimes J_{0}(W)\right)_{a} .
\end{aligned}
$$

From the exactness of (25.16) and from (25.18), we deduce that

$$
g_{1, a}=\left(\rho\left(J_{0}\left(T_{Y}\right)\right)^{*} \otimes J_{0}(V)\right)_{a} \oplus \mathfrak{a}_{1} \oplus \mathfrak{a}_{2} \oplus\left\{\sigma_{1}\left(\xi_{-t}\right)\right\} ;
$$

from the above decompositions of $h_{1}^{\prime}, h_{1}^{\prime \prime}$ and $g_{1, a}$, and by (25.27) and (25.29), we obtain the equality (25.28) with $k=1$. Furthermore, from (25.30) we see that

$$
g_{1}=a_{1}
$$


We continue to identify $W_{a}^{*}$ and $J_{0}(W)_{a}^{*}$ with subspaces of $T_{a}^{*}$ and $J_{0}(T)_{a}^{*}$ respectively as above, and for $k \geqslant 0$ let

$$
\tau_{k}: S^{k+1} W_{a}^{*} \rightarrow\left(S^{k} J_{0}(W)^{*} \otimes J_{0}(W)\right)_{a}
$$

be the mapping determined by the relation

$$
\varepsilon \tau_{k}(u)=\frac{1}{2} \sigma_{k}\left(\eta_{f}\right),
$$

if $\varepsilon u=j_{k}(f)(a)$, where $f=f(v, w)$ is a homogeneous polynomial of degree $k+1$ in $(v, w)$. If $\gamma$ denotes the inverse of the isomorphism (25.1), then it is easily seen that $\tau_{k}$ is equal to the composition

$$
S^{k+1} W_{a}^{*} \stackrel{\Delta_{k, 1}}{\longrightarrow}\left(S^{k} W^{*} \otimes W^{*}\right)_{a} \stackrel{\nu^{*-1} \otimes(\nu \circ \gamma)}{\longrightarrow}\left(S^{k} J_{0}(W)^{*} \otimes J_{0}(W)\right)_{a}
$$

and is injective; in fact, $\tau_{0}$ is equal to the isomorphism $\nu \circ \gamma$. It follows that the diagram

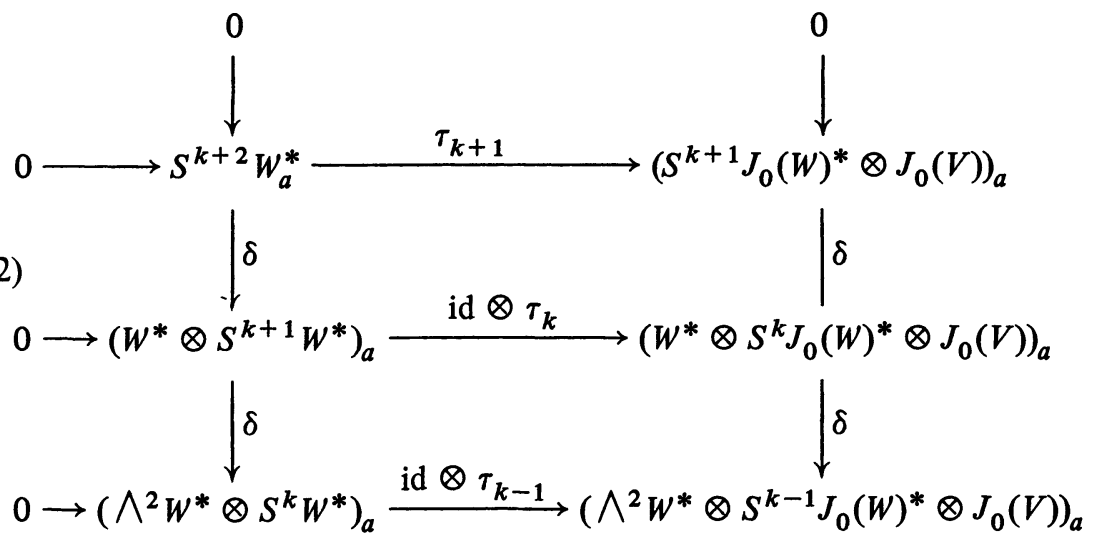

is commutative and exact. In order to complete the proof of the equalities (25.28), we now proceed to show by induction on $k$ that $g_{k}$ is equal to the image of $\tau_{k}$ for $k \geqslant 1$. The equality (25.31) says that this assertion holds for $k=1$. Assume that $\mathrm{g}_{k}$ is the image of $\tau_{k}$ for some $k \geqslant 1$. By (25.23) and [9, Lemma 6.3], $\mathfrak{g}_{k+1}$ is equal to the first prolongation of the subspace $\mathrm{g}_{k}$ of $\left(S^{k} J_{0}(W)^{*} \otimes J_{0}(V)\right)_{a}$. It follows from this remark that, if $u \in\left(S^{k+1} J_{0}(W)^{*} \otimes\right.$ $\left.J_{0}(V)\right)_{a}$, then $u$ belongs to $\mathfrak{g}_{k+1}$ if and only if $\delta u \in W_{a}^{*} \otimes \mathfrak{g}_{k}$, or equivalently if and only if there is an element $v$ of $\left(W^{*} \otimes S^{k+1} W^{*}\right)_{a}$ such that (id $\left.\otimes \tau_{k}\right) v=\delta u$. By the commutativity and exactness of diagram (25.32), this property of $v$ implies that $\delta v=0$ and so holds if and only if there is an element $w$ of $S^{k+2} W_{a}^{*}$ satisfying (id $\otimes \tau_{k}$ ) $\delta w=\delta u$; finally, this equality is equivalent to the relation $\tau_{k+1}(w)=u$, and we conclude that $g_{k+1}$ is equal to the image of $\tau_{k+1}$. 
In particular, we have just shown that

$$
\tau_{k}: S^{k+1} W_{a}^{*} \rightarrow \mathfrak{g}_{k}
$$

is an isomorphism for $k \geqslant 1$, and that $g_{k} \subset h_{k}^{\prime}$ for $k \geqslant 1$. As $\mathfrak{g}_{0}=J_{0}(V)_{a}=$ $h_{0, a}$, by (25.26) we have

$$
y^{\alpha} \cdot \mathfrak{g}_{k} \subset h_{k+|\alpha|, a},
$$

for $k \geqslant 0$. From this inclusion and (25.24), it follows that

$$
g_{k, a} \cap \operatorname{ker} \bar{\delta}_{t} \subset h_{k, a}, \quad \text { for } k \geqslant 1 .
$$

By (25.25), $\bar{\delta}_{t}$ induces a surjective mapping

$$
\overline{\delta_{t}}: h_{k+1, a} \rightarrow h_{k, a}, \quad \text { for } k \geqslant 0 \text {. }
$$

Thus if (25.28) holds for some $k \geqslant 1$, the diagram

$$
\begin{aligned}
& 0 \rightarrow g_{k+1, a} \cap \operatorname{ker} \bar{\delta}_{t} \rightarrow h_{k+1, a} \stackrel{\bar{\delta}_{t}}{\rightarrow} h_{k, a} \rightarrow 0 \\
& \qquad g_{k+1, a} \cap \operatorname{ker} \bar{\delta}_{t} \rightarrow g_{k+1, a} \stackrel{\bar{\delta}_{t}}{\rightarrow} g_{k, a} \rightarrow 0,
\end{aligned}
$$

whose vertical arrows are induced by the inclusions (25.12), is commutative and exact; hence it provides us with the equality (25.28) with $k$ replaced by $k+1$, and so (25.28) holds for all $k \geqslant 0$. From the isomorphisms (25.33) together with the commutativity of the top square of diagram (25.32), we easily see that the basis

$$
\left\{\nu\left(\frac{\partial}{\partial v^{1}}\right)_{a}, \cdots, \nu\left(\frac{\partial}{\partial v^{p}}\right)_{a}, \nu\left(\frac{\partial}{\partial w^{1}}\right)_{a}, \cdots, \nu\left(\frac{\partial}{\partial w^{p}}\right)_{a}\right\}
$$

of $J_{0}(W)_{a}$ is quasi-regular for the subspace $g_{1}$ of $\left(J_{0}(W)^{*} \otimes J_{0}(V)\right)_{a}$. Therefore from the surjectivity of (25.34), the equalities (25.28) and (25.24), we infer that the basis

$$
\begin{array}{r}
\left\{\nu\left(\frac{\partial}{\partial t}\right)_{a}, \nu\left(\frac{\partial}{\partial y^{1}}\right)_{a}, \cdots, \nu\left(\frac{\partial}{\partial y^{m}}\right)_{a}, \nu\left(\frac{\partial}{\partial v^{1}}\right)_{a}, \cdots\right. \\
\left.\nu\left(\frac{\partial}{\partial v^{p}}\right)_{a}, \nu\left(\frac{\partial}{\partial w^{1}}\right)_{a}, \cdots, \nu\left(\frac{\partial}{\partial w^{p}}\right)_{a}\right\}
\end{array}
$$

of $J_{0}(T)_{a}$ is quasi-regular for $g_{1, a}$, and so $g_{1, a}$ is involutive (see [24, Appendix]). Since every element of $h_{k, a}$ is of the form $\sigma_{k}(\xi)$, where $\xi$ is a solution of 
$R_{1}(V ; \omega)$ over $U$ satisfying $j_{k-1}(\xi)(a)=0$ if $k \geqslant 1$, from (25.28) and (25.7) we deduce that $R_{1}(V ; \omega)$ is a formally integrable and integrable Lie equation. From (25.8) and (25.10), we now obtain (25.19). Since the mappings $\pi_{k}$ : $J_{k+l}(V ; W) \rightarrow J_{k}(V ; W)$ are of constant rank for all $k, l \geqslant 1$, according to [10, Proposition 8] we have

$$
H^{j}\left(R_{1}(V ; \omega)\right)=H^{j}\left(J_{1}(V ; \omega)\right), \text { for } j \geqslant 0,
$$

and thus (25.4) gives us (25.20). From the decomposition (25.30) of $g_{1, a}$ and the definitions of $\mathfrak{a}_{1}$ and $\mathfrak{a}_{2}$, we easily see that the characteristic variety $\mathscr{V}_{a}\left(R_{1}(V ; \omega), \mathbf{C}\right)$ of $R_{1}(V ; \omega)$ over $\mathbf{C}$ is equal to $T_{a}^{*} \otimes \mathbf{C}$, concluding the proof of the proposition.

We now construct a formally integrable finite form of the Lie equation $R_{1}(V ; \omega)$. Let $\mathbf{P}\left(V^{*}\right)$ be the projectivized bundle of $V^{*}$, and denote by $[\alpha]$ the image of $\alpha \in V^{*}$, with $\alpha \neq 0$, in $\mathbf{P}\left(V^{*}\right)$. Let

$$
\Phi_{k-1}: Q_{k}(V) \rightarrow J_{k-1}\left(\mathbf{P}\left(V^{*}\right)\right)
$$

be the morphism of fibered manifolds over $X$ sending $j_{k}(f)(a)$ into $j_{k-1}\left(\left[f^{*} \omega\right]\right)(a)$, where $f$ is a local diffeomorphism of $X$ over $Y$ defined on a neighborhood of $a \in X$; the subset $Q_{k}(V ; W)$ of $Q_{k}(V)$ equal to $\Phi_{k-1}^{-1}\left(j_{k-1}([\omega])\right)$ consists of all $k$-jets $\phi=j_{k}(f)(a)$ of $Q_{k}(V)$ satisfying $j_{k-1}\left(f^{*} \omega-g \omega\right)(a)$, for some $g \in \mathcal{O}_{X, a}$, or equivalently $\phi\left(J_{k-1}(W)_{a}\right) \subset$ $J_{k-1}(W)$. Thus $Q_{k}(V ; W)$ is a sub-groupoid of $Q_{k}(V)$. We now show that it a fibered submanifold of $Q_{k}(V)$ and a finite form of the Lie equation $J_{k}(V ; W)$. If $p \in J_{k}\left(\mathbf{P}\left(V^{*}\right)\right)_{a}$ with $a \in X$ and $p=j_{k}([\alpha])(a)$, where $\alpha$ is a section of $V^{*}$ over a neighborhood of $a$ satisfying $\alpha(a) \neq 0$, then the mapping

$$
J_{k}\left(V^{*}\right)_{a} \rightarrow V_{p}\left(J_{k}\left(\mathbf{P}\left(V^{*}\right)\right)\right),
$$

sending $j_{k}(\beta)(a)$, with $\beta \in \mathcal{V}_{a}^{*}$, into

$$
\left.\frac{d}{d t} j_{k}([\alpha+t \beta])(a)\right|_{t=0},
$$

is surjective, and its kernel is the subspace of $J_{k}\left(V^{*}\right)_{a}$ generated by $j_{k}(\alpha)(a)$. Therefore if $p=j_{k}([\omega])(a)$, the projection $\varphi: V^{*} \rightarrow W^{*}$ enables us to identify $V_{p}\left(J_{k}\left(\mathbf{P}\left(V^{*}\right)\right)\right)$ with $J_{k}\left(W^{*}\right)_{a}$. If $\phi \in Q_{k}(V)$, with source $\phi=a$, target $\phi=b$, the isomorphism (25.3)

$$
\phi: J_{k-1}\left(V^{*}\right)_{b} \rightarrow J_{k-1}\left(V^{*}\right)_{a}
$$

determines a diffeomorphism

$$
\phi: J_{k-1}\left(\mathbf{P}\left(V^{*}\right)\right)_{b} \rightarrow J_{k-1}\left(\mathbf{P}\left(V^{*}\right)\right)_{a} ;
$$

in turn, this mapping induces an isomorphism

$$
\phi_{*}: V_{p}\left(J_{k-1}\left(\mathbf{P}\left(V^{*}\right)\right)\right) \rightarrow V_{\phi(p)}\left(J_{k-1}\left(\mathbf{P}\left(V^{*}\right)\right)\right),
$$


which can also be obtained by passage to the quotient from the mapping (25.35), that is, the diagram

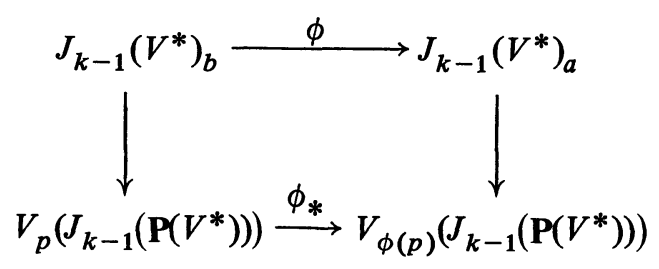

commutes. If $p=\Phi_{k-1}(\phi)=\phi\left(j_{k-1}([\omega])(b)\right)$, an argument similar to the one used to show that diagram (22.23) commutes gives us the commutativity of the diagram

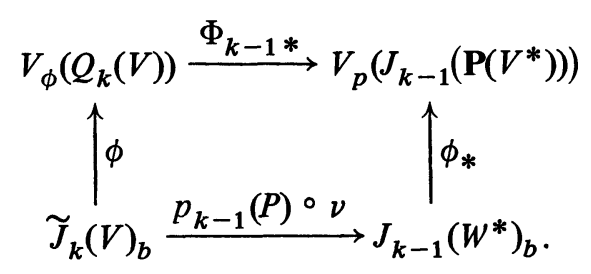

Since $J_{k}(V ; W)$ is a vector bundle, we see that $\Phi_{k-1}$ is of constant rank and by [9, Proposition 2.1] that $Q_{k}(V ; W)$ is a finite form of $J_{k}(V ; W)$. Let

$$
J_{k}\left(\Phi_{0}\right): J_{k}\left(Q_{1}(V)\right) \rightarrow J_{k}\left(\mathbf{P}\left(V^{*}\right)\right)
$$

be the morphism of fibered manifolds sending $j_{k}(\phi)(a)$ into $j_{k}\left(\Phi_{0}(\phi)\right)(a)$, where $\phi$ is a section of $Q_{1}(V)$ over a neighborhood of $a \in X$; then the diagram

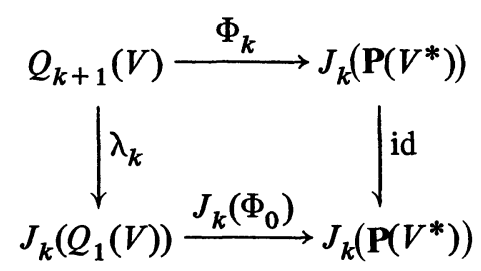

is commutative. Hence by [9, Proposition 4.4], we have

$$
\begin{aligned}
\lambda_{k} Q_{k+1}(V ; W) & =\lambda_{k} Q_{k+1}(V) \cap J_{k}\left(Q_{1}(V ; W)\right) \\
& =\lambda_{k} Q_{k+1} \cap J_{k}\left(Q_{1}(V ; W)\right),
\end{aligned}
$$

since $Q_{k+1}(V)=\left(Q_{1}(V)\right)_{+k}$; therefore $Q_{k+1}(V ; W)$ is the $k$ th prolongation of $Q_{1}(V ; W)$.

Let

$$
\Psi_{k-1}: Q_{k}(V ; W) \rightarrow J_{k-1}\left(\wedge^{2} W^{*}\right)
$$


be the morphism of fibered manifolds over $X$ sending $\phi=j_{k}(f)(a)$ into

$$
j_{k-1}\left(\left(f^{*} d_{X / Y} \omega-g d_{X / Y} \omega\right)_{\mid \wedge{ }^{2} W}\right)(a),
$$

where $f$ is a local diffeomorphism of $X$ over $Y$ defined on a neighborhood of $a \in X$ and $g$ is an element of $\vartheta_{X, a}$ satisfying $j_{k-1}\left(f^{*} \omega-g \omega\right)(a)=0$. If $b=\operatorname{target} \phi$, the isomorphism (25.3) induces by passage to the quotient an isomorphism

$$
\phi: J_{k-1}\left(\wedge W^{*}\right)_{b} \rightarrow J_{k-1}\left(\wedge W^{*}\right)_{a} ;
$$

an argument similar to the one used to show that the diagram (22.23) commutes gives us the commutativity of the diagram

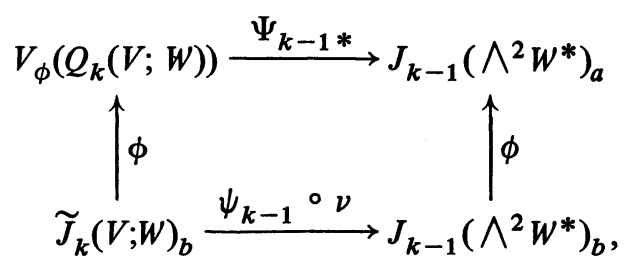

where $\tilde{J}_{k}(V ; W)=\nu^{-1} J_{k}(V ; W)$. Since $R_{k}(V ; \omega)$ is a vector bundle, we see that $\Psi_{k-1}$ is of constant rank, and by [9, Proposition 2.1] that the set $P_{k}(V ; \omega)$ consisting of all $k$-jets $\phi \in Q_{k}(V ; W)$ satisfying $\Psi_{k-1}(\phi)=0$ is a sub-groupoid and a fibered submanifold of $Q_{k}(V ; W)$. Therefore $P_{k}(V ; \omega)$ is a finite form of the Lie equation $R_{k}(V ; \omega)$, whose solutions are the local immersions $f: X \rightarrow X$ over $Y$ which satisfy $f^{*} \omega=g \omega$ for some real-valued function $g$ on $X$, or equivalently $\left(f^{*} \omega\right) \wedge \omega=0$. An element $\phi$ of $Q_{1}(V)$ belongs to $P_{1}(V ; \omega)$ if and only if there is an element $c \in \mathbf{R}$ such that

$$
\phi(\bar{\omega})=c \bar{\omega}, \quad \phi(\bar{\Omega})=c \bar{\Omega} \quad \text { on } \wedge^{2} J_{0}(W) .
$$

Let

$$
J_{k}\left(\Psi_{0}\right): J_{k}\left(Q_{1}(V ; W)\right) \rightarrow J_{k}\left(\wedge^{2} W^{*}\right)
$$

be the morphism of fibered manifolds sending $j_{k}(\phi)(a)$ into $j_{k}\left(\Psi_{0}(\phi)\right)(a)$, where $\phi$ is a section of $Q_{1}(V ; W)$ over a neighborhood of $a \in X$; then the diagram

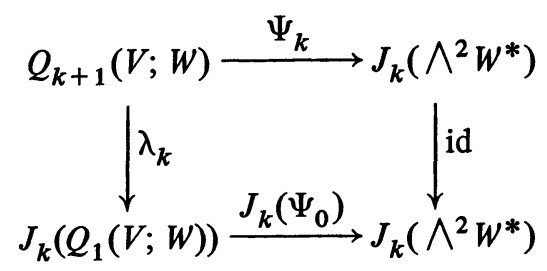


is commutative. Hence by [9, Proposition 4.4], we have

$$
\begin{aligned}
\lambda_{k} P_{k+1}(V ; \omega) & =\lambda_{k} Q_{k+1}(V ; W) \cap J_{k}\left(P_{1}(V ; \omega)\right) \\
& =\lambda_{k} Q_{k+1} \cap J_{k}\left(P_{1}(V ; \omega)\right),
\end{aligned}
$$

since $Q_{k+1}(V ; W)=\left(Q_{1}(V ; W)\right)_{+k} ;$ therefore $P_{k+1}(V ; \omega)$ is the $k$ th prolongation of $P_{1}(V ; \omega)$.

Proposition 25.3. If $\omega$ is a contact form along the fibers of $\rho$, then $P_{1}(V ; \omega)$ is a formally integrable and integrable finite form of the formally integrable Lie equation $R_{1}(V ; \omega)$, whose kth prolongation is $P_{k+1}(V ; \omega)$.

Proof. We begin by verifying that

$$
\pi_{1}: P_{2}(V ; \omega) \rightarrow P_{1}(V ; \omega)
$$

is surjective. If $a \in X$, let $G_{a}$ be the set of $\phi \in P_{1}(V ; \omega)$ with source $\phi=$ target $\phi=a$. If we take $\omega^{\prime}=\omega$ in Proposition 25.1, we see that, in order to show that the mapping $\pi_{1}$ is surjective, it is sufficient to prove that $G_{a} \subset$ $\pi_{1} P_{2}(V ; \omega)$ for all $a \in X$. Let $a \in X$ and let $(t, v, w, y)$ be the coordinate on a neighborhood $U$ of $a$ considered in the proof of Proposition 25.2. For $\lambda \neq 0$, let $h_{\lambda}$ be the local diffeomorphism of $X$ defined on a neighborhood of $a$ sending the point with coordinates $(t, v, w, y)$ into the point with coordinates

$$
\left(\lambda t, \lambda v^{1}, \cdots, \lambda v^{p}, w^{1}, \cdots, w^{p}, y\right)
$$

then $h_{\lambda}^{*} \omega=\lambda \omega$ and $h_{\lambda}(a)=a$, and so $h_{\lambda}$ is a solution of $P_{1}(V ; \omega)$. If $\phi \in G_{a}$ satisfies (25.36) for some $c \in \mathbf{R}$, then $j_{1}\left(h_{1 / c}\right)(a) \cdot \phi$ belongs to $G_{a}$ and satisfies (25.36) with $c=1$. To verify our claim, we need only show that every element $\phi$ of $G_{a}$ satisfying (25.36) with $c=1$ belongs to $\pi_{1} P_{1}(V ; \omega)$. Let $\phi \in Q_{1}(V)$ with source $\phi=$ target $\phi=a$ satisfying $\phi(\bar{\omega})=\bar{\omega}$. If $\phi: J_{0}(T)_{a} \rightarrow J_{0}(T)_{a}$ is given by

$$
\begin{aligned}
\phi\left(\nu\left(\frac{\partial}{\partial t}\right)\right) & =\nu\left(\frac{\partial}{\partial t}\right)+\sum_{l=1}^{p}\left(\lambda^{l} \nu\left(\frac{\partial}{\partial v^{l}}\right)+\mu^{l} \nu\left(\frac{\partial}{\partial w^{l}}\right)\right) \\
\phi\left(\nu\left(\frac{\partial}{\partial v^{j}}\right)\right) & =\sum_{l=1}^{p}\left(a_{j}^{l} \nu\left(\frac{\partial}{\partial v^{l}}\right)+b_{j}^{l} \nu\left(\frac{\partial}{\partial w^{l}}\right)\right), \\
\phi\left(\nu\left(\frac{\partial}{\partial w^{j}}\right)\right) & =\sum_{l=1}^{p}\left(c_{j}^{l} \nu\left(\frac{\partial}{\partial v^{l}}\right)+d_{j}^{l} \nu\left(\frac{\partial}{\partial w^{l}}\right)\right), \\
\phi\left(\nu\left(\frac{\partial}{\partial y^{i}}\right)\right) & =\nu\left(\frac{\partial}{\partial y^{i}}\right)+\alpha_{i} \nu\left(\frac{\partial}{\partial t}\right)+\sum_{l=1}^{p}\left(\beta_{i}^{l} \nu\left(\frac{\partial}{\partial v^{l}}\right)+\gamma_{i}^{l} \nu\left(\frac{\partial}{\partial w^{l}}\right)\right),
\end{aligned}
$$


with $1 \leqslant j \leqslant p, 1 \leqslant i \leqslant m$, then the mapping $f_{1}$ defined by

$$
\begin{aligned}
v^{j} \circ f_{1} & =\sum_{l=1}^{p}\left(a_{l}^{j} v^{l}+c_{l}^{j} w^{l}\right), \\
w^{j} \circ f_{1} & =\sum_{l=1}^{p}\left(b_{l}^{j} v^{l}+d_{l}^{j} w^{l}\right), \\
t \circ f_{1} & =t, \quad y^{i} \circ f_{1}=y^{i},
\end{aligned}
$$

for $1 \leqslant j \leqslant p, 1 \leqslant i \leqslant m$, is a local diffeomorphism of $X$ over $Y$ on a neighborhood of $a$; moreover it is easily verified that $f_{1}^{*} \omega=\omega$ if and only if $\phi(\bar{\Omega})=\bar{\Omega}$ on $\wedge^{2} J_{0}(W)_{a}$. Let

$$
\alpha=\sum_{j=1}^{p}\left(\lambda^{j} w^{j}-\mu^{j} v^{j}\right)
$$

the mapping $f_{2}$ defined by

$$
\begin{gathered}
v^{j} \circ f_{2}=(1+\alpha) v^{j}+\lambda^{j} t, \quad w^{j} \circ f_{2}=(1+\alpha) w^{j}+\mu^{j} t, \\
t \circ f_{2}=(1+\alpha) t, \quad y^{i} \circ f_{2}=y^{i},
\end{gathered}
$$

for $1 \leqslant j \leqslant p, 1 \leqslant i \leqslant m$, is a local diffeomorphism of $X$ over $Y$ on a neighborhood of $a$. It is easily verified that

$$
f_{2}^{*} \omega=(1+\alpha)^{2} \omega-2 t \alpha d_{X / Y} \alpha,
$$

and hence that

$$
\begin{aligned}
f_{2}^{*} d_{X / Y} \omega= & (1+\alpha)^{2} d_{X / Y} \omega+2(1+2 \alpha) d_{X / Y} \alpha \wedge \omega \\
& +2 \alpha \sum_{j=1}^{p}\left(w^{j} d_{X / Y} v^{j}-v^{j} d_{X / Y^{\prime}} w^{j}\right) \wedge d_{X / Y} \alpha .
\end{aligned}
$$

Since $\alpha(a)=0$, we have

$$
j_{1}\left(f_{2}^{*} \omega-(1+\alpha)^{2} \omega\right)(a)=0
$$

and

$$
j_{1}\left(f_{2}^{*} d_{X / Y} \omega-(1+\alpha)^{2} d_{X / Y} \omega+2(1+2 \alpha) d_{X / Y} \alpha \wedge \omega\right)(a)=0,
$$

which shows that $j_{2}\left(f_{2}\right)(a)$ belongs to $P_{2}(V ; \omega)$. Finally, the mapping $f_{3}$ defined by

$$
\begin{gathered}
v^{j} \circ f_{3}=v^{j}+\sum_{i=1}^{m} \beta_{i}^{j} y^{i}, \quad w^{j} \circ f_{3}=w^{j}+\sum_{i=1}^{m} \gamma_{i}^{j} y^{i}, \\
t \circ f_{3}=t+\sum_{i=1}^{m}\left(\alpha_{i}+\sum_{l=1}^{p}\left(\beta_{i}^{l} w^{l}-\gamma_{i}^{l} v^{l}\right)\right) y^{i}, \\
y^{i} \circ f_{3}=y^{i},
\end{gathered}
$$


for $1 \leqslant j \leqslant p, 1 \leqslant i \leqslant m$, is a local diffeomorphism of $X$ over $Y$ on a neighborhood of $a$. It is easily seen that $f_{3}^{*} \omega=\omega$ and that

$$
j_{1}\left(f_{3} \circ f_{2} \circ f_{1}\right)(a)=\phi
$$

since $\alpha(a)=0$. Thus if $\phi$ is an element of $P_{1}(V ; \omega)$, then $f_{1}$ is a solution of $P_{1}(V ; \omega)$, and so $j_{2}\left(f_{1}\right)(a)$ and $j_{2}\left(f_{3}\right)(a)$ belong to $P_{2}(V ; \omega)$; consequently $\tilde{\phi}=j_{2}\left(f_{3} \circ f_{2} \circ f_{1}\right)(a)$ is an element of $P_{2}(V ; \omega)$ satisfying $\pi_{1} \tilde{\phi}=\phi$, and so our assertion holds. Since $g_{1}$ is 2-acyclic and $g_{k}$ is a vector bundle for $k \geqslant 1$ by Proposition 25.2, we apply [9, Theorem 8.1$]$ to $P_{1}(V ; \omega)$ and deduce that it is a formally integrable finite form of $R_{1}(V ; \omega)$. With respect to the analytic structure on $U$ determined by the coordinate $(t, v, w, y)$, the form $\omega$ is analytic and the open sub-bundle of $P_{1}(V ; \omega)_{\mid U}$ consisting of all elements $\phi \in P_{1}(V ; \omega)$ with source and target belonging to $U$ is an analytic and formally integrable differential equation on $U$, and therefore integrable. That $P_{1}(V ; \omega)$ is integrable now follows from Proposition 25.1 with $\omega^{\prime}=\omega$.

The following proposition is the analogue of Proposition 22.5 (iii) for $P_{1}(V ; \omega)$ and its proof is based on Proposition 22.5 (i) and (ii).

Proposition 25.4. Let $u$ be a section of $\left(T^{*} \otimes J_{0}(V)\right)^{\wedge}$ over $X$ and let $f$ be $a$ local immersion $X \rightarrow X$ over $Y$ defined on an open set $U$. The section $\phi=$ $j_{1}(f)-f \circ \bar{u} \circ \nu$ of $\tilde{\mathscr{Q}}_{1}(V)$ is a section of $\tilde{\mathscr{P}}_{1}(V ; \omega)$ over $U$ if and only if there is a real-valued function $g$ on $U$ such that $f^{*} \omega=g \omega^{u}$ and

$$
\omega^{u} \wedge\left(f^{*} d_{X / Y} \omega-g\left(d_{X / Y} \omega\right)^{u}\right)=0 .
$$

Proof. According to Proposition 22.5 (i), $\phi$ is a section of $\tilde{Q}_{1}(V)$. Let $g$ be a real-valued function on $U$. By Proposition 22.5 (ii), with $\alpha=g \omega$, we see that $\phi(\bar{\omega})=g \bar{\omega}$ if and only if $f^{*} \omega=g \omega^{u}$; by (22.27) and (22.26), for $\xi \in \wedge^{2} J_{0}(W)$ we have

$$
\begin{gathered}
\langle\xi, \phi(\bar{\Omega})\rangle=\left\langle\left(\nu^{-1}-\bar{u}\right) \xi, f^{*} d_{X / Y} \omega\right\rangle, \\
\langle\xi, g \bar{\Omega}\rangle=\left\langle\left(\nu^{-1}-\bar{u}\right) \xi, g\left(d_{X / Y} \omega\right)^{u}\right\rangle .
\end{gathered}
$$

Since the sub-bundle $W^{u}$ of $V$ consisting of all vectors $\xi \in V$ satisfying $\left\langle\xi, \omega^{u}\right\rangle=0$ is equal to

$$
W^{u}=(\mathrm{id}-\bar{u} \circ \nu) W=\left(\mathrm{id}+\nu^{-1} \circ u\right)^{-1} W,
$$

we deduce that $\phi(\bar{\Omega})=g \bar{\Omega}$ on $\wedge^{2} J_{0}(W)$ if and only if $f^{*} d_{X / Y} \omega=g\left(d_{X / Y} \omega\right)^{u}$ on $\wedge^{2} W^{u}$; this last condition is equivalent to

$$
f^{*} d_{X / Y} \omega-g\left(d_{X / Y} \omega\right)^{u}=\alpha \wedge \omega^{u},
$$


for some section $\alpha$ of $V^{*}$ over $U$, and hence to (25.37). The desired result is now a consequence of the description (25.36) of $P_{1}(V ; \omega)$.

If $u$ is a section of $V^{*} \otimes J_{0}(V)$ and $v$ is a section of $\wedge J_{0}(V)^{*} \otimes \tilde{J}_{1}(V ; W)$, we have the relation

$$
\left(\varrho_{u}(v) \bar{\omega}\right) \wedge \nu^{*-1} \omega^{u}=0,
$$

which we require for the next proposition. Indeed, if $v=\alpha \otimes \tilde{\xi}$, with $\alpha$ a section of $\wedge J_{0}(V)^{*}$ and $\tilde{\xi}$ a section of $\tilde{J}_{1}(V ; W)$, then

$$
\begin{aligned}
\left(\varrho_{u}(v) \bar{\omega}\right) \wedge \nu^{*-1} \omega^{u} & =\alpha \wedge(\mathcal{E}(\tilde{\xi}) \bar{\omega})^{u} \wedge \nu^{*-1} \omega^{u} \\
& =\alpha \wedge((\mathcal{L}(\tilde{\xi}) \bar{\omega}) \wedge \bar{\omega})^{u}=0 .
\end{aligned}
$$

The following two propositions are the analogues of Propositions 23.2 and 23.4 or of Proposition 24.4 for contact forms.

Proposition 25.5. Let $\omega$ be a contact form along the fibers of $\rho$ and $u_{0}$ be $a$ section of $\left(T^{*} \otimes J_{0}(V)\right)^{\wedge}$ over $X$. If $a \in X$, the following assertions are equivalent:

(i) $\omega^{u_{0}}$ is a contact form along the fibers of $\rho$ and

$$
\omega^{u_{0}} \wedge\left(d_{X / Y} \omega^{u_{0}}-\left(d_{X / Y} \omega\right)^{u_{0}}\right)=0
$$

on a neighborhood of a;

(ii) relation (25.39) holds on a neighborhood of $a$;

(iii) there is a section $u$ of $\left(T^{*} \otimes R_{1}(V ; \omega)\right)^{\wedge}$ such that $\pi_{0} u=u_{0}$ and $\mathfrak{D}_{1} u=0$ on a neighborhood of $a$;

(iv) there is a section $\phi$ of $\tilde{\mathscr{P}}_{1}(V ; \omega)$ over a neighborhood of a satisfying $\mathscr{D} \phi=u_{0}$;

(v) there is a local diffeomorphism $f: X \rightarrow X$ over $Y$ defined on a neighborhood of a such that

$$
f^{*} \omega=\omega^{u_{0}}, \quad \omega^{u_{0}} \wedge\left(f^{*} d_{X / Y} \omega-\left(d_{X / Y} \omega\right)^{u_{0}}\right)=0
$$

(vi) there is a local diffeomorphism $f: X \rightarrow X$ over $Y$ defined on a neighborhood of $a$ such that $f(a)=a$ and $f^{*} \omega=\omega^{u_{0}}$, and (25.39) holds on a neighborhood of $a$.

Proof. (iii) $\Rightarrow$ (ii): Let $u$ be a section of $T^{*} \otimes J_{1}(V ; W)$ satisfying $\pi_{0} u=u_{0}$ and $\mathfrak{D}_{1} u=0$; then $\hat{u}=\left(\nu^{*-1} \otimes \nu^{-1}\right)\left(u_{\mid V}\right)$ is a section of $J_{0}(V)^{*} \otimes \tilde{J}_{1}(V ; W)$. Since $\mathfrak{D}_{1, X / Y}\left(u_{\mid V}\right)=0$, we apply Proposition 22.3 to $\omega$ and $u_{\mid V}$ and obtain

$$
\omega^{u_{0}} \wedge\left(d_{X / Y} \omega^{u_{0}}-\left(d_{X / Y} \omega\right)^{u_{0}}\right)=-\omega^{u_{0}} \wedge \nu^{*} \varrho_{v}(\hat{u}) \bar{\omega}=0,
$$

by (25.38), where $v=u_{0 \mid V}$. 
(ii) $\Rightarrow$ (i): If (25.39) holds on an open set $U$, then

$$
\begin{aligned}
\omega^{u_{0}} \wedge\left(d_{X / Y} \omega^{u_{0}}\right)^{p} & =\omega^{u_{0}} \wedge\left(\left(d_{X / Y} \omega\right)^{u_{0}}+\left(d_{X / Y} \omega^{u_{0}}-\left(d_{X / Y} \omega\right)^{u_{0}}\right)\right)^{p} \\
& =\omega^{u_{0}} \wedge\left(\left(d_{X / Y} \omega\right)^{u_{0}}\right)^{p}=\left(\omega^{u_{0}} \wedge\left(d_{X / Y} \omega\right)^{p}\right)^{u_{0}}
\end{aligned}
$$

Since id $+\nu^{-1} \circ u_{0}: V \rightarrow V$ is invertible, $\left(\omega \wedge\left(d_{X / Y} \omega\right)^{p}\right)^{u_{0}}$, and hence also $\omega^{u_{0}} \wedge\left(d_{X / Y} \omega^{u_{0}}\right)^{p}$, does not vanish at any point of $U$. Thus $\omega^{u_{0}}$ is a contact form on $U$.

(i) $\Rightarrow$ (vi) is given by Proposition 25.1 .

(vi) $\Rightarrow(v)$ : Since $f^{*} \omega=\omega^{u_{0}}$, we have $f^{*} d_{X / Y} \omega=d_{X / Y} \omega^{u_{0}}$ and the desired identity follows from (25.39).

(v) $\Rightarrow$ (iv) follows from Propositions 25.4 and 22.5 (i).

(iv) $\Rightarrow$ (iii): Since $P_{1}(V ; \omega)$ is a formally integrable finite form of $R_{1}(V ; \omega)$ by Proposition 25.3 , there is a section $\psi$ of $\tilde{\mathscr{P}}_{2}(V ; \omega)$ over a neighborhood of $a$ such that $\pi_{1} \psi=\phi$; then $u=\mathfrak{D} \psi$ is a section of $\left(T^{*} \otimes R_{1}(V ; \omega)\right)^{\wedge}$ satisfying $\pi_{0} u=u_{0}$ and $\mathfrak{D}_{1} u=0$.

The following proposition, together with the equivalence of assertions (i) and (iii) of Proposition 25.5, shows that the mapping $T^{*} \otimes J_{1}(V) \rightarrow V^{*}$, sending $u$ into $\omega^{u_{0}}$, with $u_{0}=\pi_{0} u$, induces a surjective mapping from $Z^{1}\left(R_{1}(V ; \omega)\right)_{a}$ to the set of germs at $a \in X$ of contact forms along the fibers of $\rho$.

Proposition 25.6. Let $\omega$ be a contact form along the fibers of $\rho$ and $\omega^{\prime} a$ section of $V^{*}$ over $X$. Then $\omega^{\prime}$ is a contact form along the fibers of $\rho$ if and only if, for all $a \in X$, there is a section $u_{0}$ of $\left(T^{*} \otimes J_{0}(V)\right)^{\wedge}$ such that $\omega^{\prime}=\omega^{u_{0}}$ and

$$
\omega^{\prime} \wedge\left(d_{X / Y} \omega^{\prime}-\left(d_{X / Y} \omega\right)^{u_{0}}\right)=0
$$

on a neighborhood of $a$.

Proof. Assume that $\omega^{\prime}$ is a contact form along the fibers of $\rho$. Let $W^{\prime}$ be the sub-bundle of $V$ consisting of all elements $\xi \in V$ satisfying $\left\langle\xi, \omega^{\prime}\right\rangle=0$. According to Cartan's lemma, there are frames $\left\{\alpha_{1}, \cdots, \alpha_{2 p}\right\}$ for $W^{*}$ and $\left\{\alpha_{1}^{\prime}, \cdots, \alpha_{2 p}^{\prime}\right\}$ for $W^{\prime *}$ over a neighborhood $U$ of $a \in X$ such that

$$
\left(d_{X / Y} \omega\right)_{\mid \wedge{ }^{2} W}=\sum_{j=1}^{p} \alpha_{2 j-1} \wedge \alpha_{2 j}, \quad\left(d_{X / Y} \omega^{\prime}\right)_{\mid \wedge{ }^{2} W^{\prime}}=\sum_{j=1}^{p} \alpha_{2 j-1}^{\prime} \wedge \alpha_{2 j}^{\prime},
$$

and there are sections $\zeta, \zeta^{\prime}$ of $V$ over $U$ such that $\langle\zeta, \omega\rangle=1,\left\langle\zeta^{\prime}, \omega^{\prime}\right\rangle=1$. Let $v$ be the unique section of $V^{*} \otimes V$ over $U$ satisfying $v\left(\zeta^{\prime}\right)=\zeta$ and whose restriction to $W^{\prime}$ is the section $\bar{v}$ of $W^{* *} \otimes W$ determined by $\bar{v}^{*}\left(\alpha_{j}\right)=\alpha_{j}^{\prime}$, for $1 \leqslant j \leqslant 2 p$. Let $u_{0}$ be a section of $T^{*} \otimes J_{0}(V)$ over $U$ satisfying (id $\left.+\nu^{-1} \circ u_{0}\right)_{\mid V}=v$; since $\nu+u_{0}: V \rightarrow J_{0}(V)$ is an isomorphism, $u_{0}$ is a section of $\left(T^{*} \otimes J_{0}(V)\right)^{\wedge}$. Then $\omega^{\prime}=\omega^{u_{0}}$ and $d_{X / Y} \omega^{\prime}=\left(d_{X / Y} \omega\right)^{u_{0}}$ on $\wedge^{2} W^{\prime}$; 
hence (25.40) holds on $U$. The converse is provided by the implication (ii) $\Rightarrow$ (i) of Proposition 25.5.

Since $P_{1}(V ; \omega)$ is a formally integrable and integrable finite form of the Lie equation $R_{1}(V ; \omega)$ by Proposition 25.3, the implication (iii) $\Rightarrow$ (iv) of Proposition 25.5 together with Proposition 22.7 (iii) gives us the following:

Theorem 25.1. Let $\omega$ be a contact form along the fibers of $\rho$. Then for all $m \geqslant 1, a \in X$, we have

$$
\bar{H}^{1}\left(P_{1}(V ; \omega)\right)_{m, a}=0, \quad \tilde{H}^{1}\left(R_{1}(V ; \omega)\right)_{a}=0 .
$$

The existence of the local coordinates for $X$ satisfying the conditions with respect to $\omega$ described in the proof of Proposition 25.1 shows that sequence

$$
0 \rightarrow R_{k}(V ; \omega)_{x} \rightarrow R_{k}(\omega)_{x} \stackrel{\rho}{\rightarrow} J_{k}\left(T_{Y} ; Y\right)_{\rho(x)} \rightarrow 0
$$

is exact, for $x \in X$ and $1 \leqslant k \leqslant \infty$; therefore by Proposition 25.2 and (25.7), we see that $R_{1}(\omega)$ is a formally transitive and formally integrable $\rho$-projectable Lie equation whose $k$ th prolongation is $R_{k+1}(\omega)$. From (25.6), we deduce that

$$
\left[\tilde{\Re}_{2}(\omega), \Re_{1}(V ; \omega)\right] \subset \Re_{1}(V ; \omega),
$$

if $\tilde{R}_{2}(\omega)=\nu^{-1} R_{2}(\omega)$, and that $R_{\infty}(V ; \omega)_{x}$ is a closed ideal of the transitive Lie algebra $R_{\infty}(\omega)_{x}$, for $x \in X$. With respect to the structure of analytic manifold on an open set $U \subset X$ determined by a local coordinate on $U$ satisfying the conditions with respect to $\omega$ described in the proof of Proposition 25.1, the section $\omega$ is analytic and the Lie equations $R_{1}(V ; \omega)$ and $R_{1}(\omega)$ are analytic and satisfy (25.41); hence for $x \in X$ and $j>0$, we have

$$
\begin{aligned}
& H^{j}\left(R_{\infty}(\omega)_{x}, R_{\infty}(V ; \omega)_{x}\right)=H^{j}\left(R_{1}(V ; \omega)\right)_{x}=0, \\
& \tilde{H}^{1}\left(R_{\infty}(\omega)_{x}, R_{\infty}(V ; \omega)_{x}\right)=\tilde{H}^{1}\left(R_{1}(V ; \omega)\right)_{x}=0,
\end{aligned}
$$

by (25.20) and Theorem 25.1; moreover by [16, Corollary 13.1] and Corollary 10.1 , we obtain

$$
\begin{aligned}
& H^{j}\left(R_{\infty}(\omega)_{x}\right)=H^{j}\left(R_{1}(\omega)\right)_{x}=0, \\
& \tilde{H}^{1}\left(R_{\infty}(\omega)\right)_{x}=\tilde{H}^{1}\left(R_{1}(\omega)\right)_{x}=0,
\end{aligned}
$$

for all $x \in X$ and $j>0$. According to Proposition 25.2 and Theorem 16.4 (i), the characteristic variety $\mathcal{V}\left(R_{\infty}(\omega)_{x}, R_{\infty}(V ; \omega)_{x}, \mathrm{C}\right)$ is equal to the image of the injective mapping

$$
\pi_{0}^{*} \circ \nu^{*-1} \otimes \mathrm{id}: T_{x}^{*} \otimes \mathrm{C} \rightarrow R_{\infty}(\omega)_{x}^{*} \otimes \mathrm{C} .
$$

If the manifold $Y$ consists of just one point, then $V=T$ and $\omega$ is a contact form on $X$. Applying the above results to $\omega$, we see that $R_{1}(\omega)=R_{1}(T ; \omega)$ is a formally transitive and formally integrable Lie equation on $X$. The solutions of 
$R_{1}(\omega)$ are the contact vector fields, $R_{\infty}(\omega)_{x}$ is the contact algebra for $x \in X$, and the local diffeomorphisms solutions of $P_{1}(T ; \omega)$ are the contact transformations of $X$. The transitive Lie algebra $R_{\infty}(\omega)_{x}$ is non-abelian, simple and infinite-dimensional; moreover if $\operatorname{Der}\left(R_{\infty}(\omega)_{x}\right)$ denotes the Lie algebra of derivations of $R_{\infty}(\omega)_{x}$, according to [7] the mapping

$$
R_{\infty}(\omega)_{x} \rightarrow \operatorname{Der}\left(R_{\infty}(\omega)_{x}\right)
$$

sending $\xi$ into the derivation $\operatorname{ad}(\xi)$ of $R_{\infty}(\omega)_{x}$, is an isomorphism of Lie algebras (see [23, §7] and [6, Corollary 2.2]).

We no longer assume that $Y$ is a point. Let $b \in Y$ and $Z$ be the submanifold $X_{b}$ of $X$. We consider the contact form $\omega_{Z}$, restriction of $\omega$ to $Z$. If $a \in Z$, a local coordinate on a neighborhood of $a$ satisfying the conditions with respect to $\omega$ described in the proof of Proposition 25.1 determines a local isomorphism $\varphi: Y \times Z \rightarrow X$ of fibered manifolds over $Y$ defined on a neighborhood of $(b, a)$ such that $\varphi(b, a)=a$ and $\tilde{\omega}_{Z}=\varphi^{*}(\omega)$. If $y \in Y, z \in Z$, with $\varphi(y, z)=$ $x$, the mapping $\varphi$ induces an isomorphism (21.4) of transitive Lie algebras; under this isomorphism, it is easily seen that the image of the semi-direct product

$$
M=\left(R_{\infty}\left(\omega_{Z}\right)_{z} \hat{\otimes} F_{y}\right) \oplus J_{\infty}\left(T_{Y} ; Y\right)_{y}
$$

is the transitive subalgebra $R_{\infty}(\omega)_{x}$ of $J_{\infty}(T ; \rho)_{x}$, and that the image of the closed ideal $R_{\infty}\left(\omega_{z}\right)_{z} \hat{\otimes} F_{y}$ of $M$ is the closed ideal $R_{\infty}(V ; \omega)_{x}$ of $R_{\infty}(\omega)_{x}$. Since $R_{\infty}\left(\omega_{z}\right)_{z}$ is a non-abelian simple Lie algebra, $R_{\infty}\left(\omega_{z}\right)_{z} \hat{\otimes} F_{y}$ and $R_{\infty}(V ; \omega)_{x}$ are non-abelian minimal closed ideals of $M$ and $R_{\infty}(\omega)_{x}$ respectively; moreover

$$
\begin{gathered}
M \supset R_{\infty}\left(\omega_{z}\right)_{z} \hat{\otimes} F_{y} \supset 0, \\
R_{\infty}(\omega)_{x} \supset R_{\infty}(V ; \omega)_{x} \supset 0
\end{gathered}
$$

are Jordan-Hölder sequences for the transitive Lie algebras $M$ and $R_{\infty}(V ; \omega)_{x}$ respectively. From the commutativity of diagram (21.6), we deduce that the characteristic variety $\mathscr{V}\left(M, R_{\infty}\left(\omega_{z}\right)_{z} \hat{\otimes} F_{y}, \mathrm{C}\right)$ of the closed ideal $R_{\infty}\left(\omega_{z}\right)_{z} \hat{\otimes} F_{y}$ of $M$ over $C$ is equal to the image of the injective mapping (21.7), with $L=R_{\infty}\left(\omega_{Z}\right)_{z}$.

We restate some of these results in

Proposition 25.7. Let $\omega$ be a contact form along the fibers of $\rho: X \rightarrow Y$. Let $x \in X$ with $y=\rho(x)$, and $Z=\rho^{-1}(x)$. If $\omega_{Z}$ is the contact form on $Z$, restriction of $\omega$ to $Z$, then the contact algebra $R_{\infty}\left(\omega_{Z}\right)_{x}$ is a transitive subalgebra of $J_{\infty}\left(T_{Z} ; Z\right)_{x}$; moreover, there is an isomorphism of transitive Lie algebras

$$
\left(R_{\infty}\left(\omega_{Z}\right)_{x} \hat{\otimes} F_{y}\right) \oplus J_{\infty}\left(T_{Y} ; Y\right)_{y} \rightarrow R_{\infty}(\omega)_{x}
$$


mapping the closed ideal $R_{\infty}\left(\omega_{Z}\right)_{z} \hat{\otimes} F_{y}$ of the semi-direct product onto the closed ideal $R_{\infty}(V ; \omega)_{x}$ of $R_{\infty}(\omega)_{x}$.

Let $\omega_{Z}$ be a contact form on a manifold $Z$. Let $\rho: X \rightarrow Y$ be the fibered manifold $\mathrm{pr}_{1}: Y \times Z \rightarrow Y$ and $\omega$ be the contact form $\tilde{\omega}_{Z}$ along the fibers of $\rho$ determined by $\omega_{Z}$. We have the decompositions

$$
R_{k}(\omega)=R_{k}(V ; \omega) \oplus J_{k}\left(V^{\prime} ; \rho\right),
$$

and, for $x=(y, z) \in Y \times Z$,

$$
R_{\infty}(\omega)_{x}=R_{\infty}(V ; \omega)_{x} \oplus \sigma_{x}\left(J_{\infty}\left(T_{Y} ; Y\right)_{y}\right) .
$$

Therefore $R_{\infty}(V ; \omega)_{x}$ is a $J_{\infty}\left(T_{Y} ; Y\right)_{y}$-submodule of $J_{\infty}(V)_{x}$, and the restriction

$$
\lambda: R_{\infty}\left(\omega_{Z}\right)_{z} \hat{\otimes} F_{y} \rightarrow R_{\infty}(V ; \omega)_{x}
$$

of the mapping (21.3) is an isomorphism of $J_{\infty}\left(T_{Y} ; Y\right)_{y}$-modules.

From (25.42) and Proposition 25.7, we deduce the following:

Theorem 25.2. If $\omega_{Z}$ is a contact form on a manifold $Z$, then for $z \in Z$, $y \in Y$ and $j>0$, we have

$$
\begin{aligned}
& H^{j}\left(\left(R_{\infty}\left(\omega_{z}\right)_{z} \hat{\otimes} F_{y}\right) \oplus J_{\infty}\left(T_{Y} ; Y\right)_{y}, R_{\infty}\left(\omega_{Z}\right)_{z} \hat{\otimes} F_{y}\right)=0, \\
& \tilde{H}^{1}\left(\left(R_{\infty}\left(\omega_{z}\right)_{z} \hat{\otimes} F_{y}\right) \oplus J_{\infty}\left(T_{Y} ; Y\right)_{y}, R_{\infty}\left(\omega_{Z}\right)_{z} \hat{\otimes} F_{y}\right)=0 .
\end{aligned}
$$

If we take $Y$ to be a point and $Z=X$ in Theorem 25.2, we obtain:

Corollary 25.1. If $\omega$ is a contact form on $X$, then for $x \in X$ and $j>0$, we have

$$
H^{j}\left(R_{\infty}(\omega)_{x}\right)=0, \quad \tilde{H}^{1}\left(R_{\infty}(\omega)_{x}\right)=0
$$

26. The cohomology of non-abelian minimal closed ideals of real type

Let $R$ be a simple real transitive Lie algebra. The commutator ring $K_{R}$ of $R$ is the algebra of all $\mathbf{R}$-linear mappings $c: R \rightarrow R$ such that

$$
c([\xi, \eta])=[\xi, c(\eta)],
$$

for $\xi, \eta \in R$. If $R$ is non-abelian, then according to [20, Proposition 4.4] the ring $K_{R}$ is a finite algebraic extension of $\mathbf{R}$; therefore $K_{R}$ is always a field which is either $\mathbf{R}$ or $\mathbf{C}$.

If $\operatorname{Der}(R)$ denotes the Lie algebra of derivations of $R$, we may identify $R$ with the closed ideal of $\operatorname{Der}(R)$ of inner derivations of $R$. If $R$ is finite-dimensional, it is a classical result that $\operatorname{Der}(R)=R$, and we let $R^{0}$ be any proper subalgebra of $R$. According to the classification of the real simple infinitedimensional transitive Lie algebras (see [34] and [36]) and the computation of 
their Lie algebra of derivations given in [7], if $R$ is infinite-dimensional and $K_{R}=\mathbf{R}$, there is an isomorphism of pairs of Lie algebras from $(\operatorname{Der}(R), R)$ to one of the following pairs of transitive Lie algebras, whose restriction to $R$ is an isomorphism of transitive Lie algebras:

(i) $\left(J_{\infty}\left(T_{Z} ; Z\right)_{z}, J_{\infty}\left(T_{Z} ; Z\right)_{z}\right)$, where $Z$ is a manifold of dimension $\geqslant 1$ and $z \in Z$;

(ii) $\left(J_{\infty}^{\prime}\left(T_{Z} ; \omega_{Z}\right)_{z}, J_{\infty}\left(T_{Z} ; \omega_{Z}\right)_{z}\right)$, where $\omega_{Z}$ is a volume form on a manifold $Z$ of dimension $\geqslant 2$ and $z \in Z$;

(iii) $\left(J_{\infty}^{\prime}\left(T_{Z} ; \omega_{Z}\right)_{z}, J_{\infty}\left(T_{Z} ; \omega_{Z}\right)_{z}\right)$, where $\omega_{Z}$ is a symplectic form on a manifold $Z$ of dimension $\geqslant 2$ and $z \in Z$;

(iv) $\left(R_{\infty}\left(\omega_{Z}\right)_{z}, R_{\infty}\left(\omega_{Z}\right)_{z}\right)$, where $\omega_{Z}$ is a contact form on a manifold $Z$ of dimension $\geqslant 3$ and $z \in Z$.

This isomorphism endows $\operatorname{Der}(R)$ with the structure of transitive Lie algebra in which the transitive Lie algebra $R$ is a closed ideal of codimension at most one. In all four cases, the images of $\operatorname{Der}(R)$ and $R$ under this isomorphism are transitive subalgebras of $J_{\infty}\left(T_{Z} ; Z\right)_{z}$ and so clearly the subalgebra $\operatorname{Der}^{0}(R)$ of $\operatorname{Der}(R)$, whose image under this isomorphism is equal to the intersection of the image of $\operatorname{Der}(R)$ with $J_{\infty}^{0}\left(T_{Z} ; Z\right)_{z}$, is a fundamental subalgebra of $\operatorname{Der}(R)$ such that $R^{0}=R \cap \operatorname{Der}^{0}(R)$ is a fundamental subalgebra of $R$, and

$$
\operatorname{Der}(R)=R+\operatorname{Der}^{0}(R)
$$

(see [6, Corollary 2.2]).

If $F_{y}=J_{\infty}(1 ; Y)_{y}$, with $y \in Y$, as we have seen in $\S 21$, the semi-direct product

$$
\operatorname{Der}\left(R \hat{\otimes}_{\mathbf{R}} F_{y}\right)=\left(\operatorname{Der}(R) \hat{\otimes}_{\mathbf{R}} F_{y}\right) \oplus J_{\infty}\left(T_{Y} ; Y\right)_{y}
$$

is a transitive Lie algebra, and

$$
\operatorname{Der}^{0}\left(R \hat{\otimes}_{\mathbf{R}} F_{y}\right)=\left(\operatorname{Der}^{0}(R) \hat{\otimes}_{\mathbf{R}} F_{y}+\operatorname{Der}(R) \hat{\otimes}_{\mathbf{R}} F_{y}^{0}\right) \oplus J_{\infty}^{0}\left(T_{Y} ; Y\right)_{y}
$$

is a fundamental subalgebra of $\operatorname{Der}\left(R \hat{\otimes}_{\mathbf{R}} F_{y}\right)$; moreover the closed ideal $R \hat{\otimes}_{\mathbf{R}} F_{y}$ of $\operatorname{Der}\left(R \hat{\otimes}_{\mathbf{R}} F_{y}\right)$ is a non-abelian minimal closed ideal if $R$ is non-abelian. We denote by

$$
\pi: \operatorname{Der}\left(R \hat{\otimes}_{\mathbf{R}} F_{y}\right) \rightarrow J_{\infty}\left(T_{Y} ; Y\right)_{y}
$$

the natural morphism of transitive Lie algebras. In fact if $K_{R}=\mathbf{R}$, then by [6, Proposition 3.2], $\operatorname{Der}\left(R \hat{\otimes}_{\mathbf{R}} F_{y}\right)$ is canonically isomorphic to the Lie algebra of derivations of $R \hat{\otimes}_{\mathbf{R}} F_{y}$, although this fact is used neither in this paper nor in the proofs of any of the results of [6] which we need. 
From the above classification results, Proposition 22.10 and Theorems 23.2, 24.2 and 25.2, we deduce:

Theorem 26.1. Let $R$ be a simple real transitive Lie algebra with $K_{R}=\mathbf{R}$. Then for $y \in Y$, we have

for $j>0$ and

$$
H^{j}\left(\operatorname{Der}\left(R \hat{\otimes}_{\mathbf{R}} F_{y}\right), R \hat{\otimes}_{\mathbf{R}} F_{y}\right)=0
$$

$$
\tilde{H}^{1}\left(\operatorname{Der}\left(R \hat{\otimes}_{\mathbf{R}} F_{y}\right), R \hat{\otimes}_{\mathbf{R}} F_{y}\right)=0 .
$$

Moreover according to the computation of characteristic varieties of $\S \S 21$, 23, 24 and 25, we see that $R \hat{\otimes}_{\mathbf{R}} F_{y}$ is an elliptic ideal of $\operatorname{Der}\left(R \hat{\otimes}_{\mathbf{R}} F_{y}\right)$ if and only if $R$ is finite-dimensional and $Y$ is zero-dimensional (i.e., $F_{y}=\mathbf{R}$ ).

Let $L$ be a real transitive Lie algebra and $I$ a non-abelian minimal closed ideal of $L$. Then according to [20, Proposition 7.1], $I$ possesses a unique maximal closed ideal $J$ of $I$ and $R=I / J$ is a non-abelian simple transitive Lie algebra which is canonically associated to $I$. We say that the non-abelian minimal closed ideal $I$ is of real or complex type according to whether the commutator field $K_{R}$ is $\mathbf{R}$ or $\mathbf{C}$.

Assume that the ideal $I$ is of real type. We now prove that the non-linear cohomology of $I$ vanishes following the argument given in $\$ 13$. We endow $\operatorname{Der}(R)$ with a structure of transitive Lie algebra and let $\operatorname{Der}^{0}(R)$ be a fundamental subalgebra of $\operatorname{Der}(R)$ satisfying the conditions described above. By [20, Proposition 6.2], the normalizer $N$ of $J$ in $L$ is an open subalgebra of $L$, and is therefore of finite codimension in $L$. We now suppose that the dimension of the manifold $Y$ is equal to that of $L / N$, and let $y \in Y$. By Proposition 21.1, there is a transitive representation

$$
\phi:(L, N) \rightarrow\left(J_{\infty}\left(T_{Y} ; Y\right)_{y}, J_{\infty}^{0}\left(T_{Y} ; Y\right)_{y}\right)
$$

of $(L, N)$ on $J_{\infty}\left(T_{Y} ; Y\right)_{y}$. Consider the transitive Lie algebra $\operatorname{Der}\left(R \hat{\otimes}_{\mathbf{R}} F_{y}\right)$ and its fundamental subalgebra $\operatorname{Der}^{0}\left(R \hat{\otimes}_{\mathbf{R}} F_{y}\right)$. According to the topological version [6, Theorem 4.2] of the structure theorem of Guillemin [20, Theorem 7.1], there is a continuous morphism of transitive Lie algebras

$$
\Phi: L \rightarrow \operatorname{Der}\left(R \hat{\otimes}_{\mathbf{R}} F_{y}\right),
$$

which induces by restriction an isomorphism

$$
\Phi: I \rightarrow R \hat{\otimes}_{\mathbf{R}} F_{y}
$$

of closed ideals, such that $\pi \circ \Phi=\phi$. Since $R \hat{\otimes}_{\mathbf{R}} F_{y}$ is contained in $\Phi(L)$ and (26.1) holds and since $\phi(L)$ is a transitive subalgebra of $J_{\infty}\left(T_{Y} ; Y\right)_{y}$, we see that $\Phi(L)$ is a transitive Lie algebra satisfying

$$
\operatorname{Der}\left(R \hat{\otimes}_{\mathbf{R}} F_{y}\right)=\Phi(L)+\operatorname{Der}^{0}\left(R \hat{\otimes}_{\mathbf{R}} F_{y}\right) .
$$


Since (26.2) is an isomorphism, by [16, Corollary 13.1 (ii)] and Theorem 10.4 (iv), $\Phi$ induces isomorphisms of cohomology

$$
\begin{aligned}
H^{*}(L, I) & \rightarrow H^{*}\left(\Phi(L), R \hat{\otimes}_{\mathbf{R}} F_{y}\right), \\
\tilde{H}^{1}(L, I) & \rightarrow \tilde{H}^{1}\left(\Phi(L), R \hat{\otimes}_{\mathbf{R}} F_{y}\right) .
\end{aligned}
$$

Because of (26.3), from [16, Theorem 13.2] and Theorem 10.5 we obtain isomorphisms of cohomology

$$
\begin{aligned}
& H^{*}\left(\Phi(L), R \hat{\otimes}_{\mathbf{R}} F_{y}\right) \rightarrow H^{*}\left(\operatorname{Der}\left(R \hat{\otimes}_{\mathbf{R}} F_{y}\right), R \hat{\otimes}_{\mathbf{R}} F_{y}\right), \\
& \tilde{H}^{1}\left(\Phi(L), R \hat{\otimes}_{\mathbf{R}} F_{y}\right) \rightarrow \tilde{H}^{1}\left(\operatorname{Der}\left(R \hat{\otimes}_{\mathbf{R}} F_{y}\right), R \hat{\otimes}_{\mathbf{R}} F_{y}\right) .
\end{aligned}
$$

Composing these isomorphisms, we obtain isomorphisms of cohomology

$$
\begin{aligned}
& H^{*}(L, I) \rightarrow H^{*}\left(\operatorname{Der}\left(R \hat{\otimes}_{\mathbf{R}} F_{y}\right), R \hat{\otimes}_{\mathbf{R}} F_{y}\right), \\
& \tilde{H}^{1}(L, I) \rightarrow \tilde{H}^{1}\left(\operatorname{Der}\left(R \hat{\otimes}_{\mathbf{R}} F_{y}\right), R \hat{\otimes}_{\mathbf{R}} F_{y}\right) .
\end{aligned}
$$

From Theorem 26.1, we deduce that Conjecture I of $\$ 13$ holds for non-abelian minimal closed ideals of real type, and so we have:

Theorem 26.2. Let $L$ be a real transitive Lie algebra and I a non-abelian minimal closed ideal of real type. Then for $j>0$, we have

$$
H^{j}(L, I)=0, \quad \tilde{H}^{1}(L, I)=0 .
$$

Furthermore, if $K=\mathbf{R}$ or $\mathbf{C}$, since (26.2) is an isomorphism, Corollary 16.1 tells us that

$$
\mathcal{V}(L, I, K)=\left(\Phi^{*} \otimes \mathrm{id}\right)\left(\mathcal{V}\left(\Phi(L), R \hat{\otimes}_{\mathrm{R}} F_{y}, K\right)\right),
$$

where $\Phi^{*} \otimes$ id: $\Phi(L)^{*} \otimes K \rightarrow L^{*} \otimes K$ is the injective mapping induced by $\Phi$. By (26.3), if we compute the characteristic varieties of the closed ideal $R \hat{\otimes}_{\mathbf{R}} F_{y}$ of $\operatorname{Der}\left(R \hat{\otimes}_{\mathbf{R}} F_{y}\right)$ and of $\Phi(L)$ using the fundamental subspace

$$
\left(R \hat{\otimes}_{\mathbf{R}} F_{y}\right) \cap \operatorname{Der}^{0}\left(R \hat{\otimes}_{\mathbf{R}} F_{y}\right)
$$

of $\mathbf{R} \hat{\otimes} F_{y}$ and the fundamental subalgebras $\operatorname{Der}^{0}\left(R \hat{\otimes}_{\mathbf{R}} F_{y}\right)$ and $\Phi(L) \cap$ $\operatorname{Der}^{0}\left(R \hat{\otimes}_{\mathbf{R}} F_{y}\right)$ of $\operatorname{Der}\left(R \hat{\otimes}_{\mathbf{R}} F_{y}\right)$ and $\Phi(L)$, we see that the mapping

$$
\left(\operatorname{Der}\left(R \hat{\otimes}_{\mathrm{R}} F_{y}\right)\right)^{*} \rightarrow \Phi(L) *
$$

induces a bijective mapping

$$
\mathcal{V}\left(\operatorname{Der}\left(R \hat{\otimes}_{\mathbf{R}} F_{y}\right), R \hat{\otimes}_{\mathbf{R}} F_{y}, K\right) \rightarrow \mathcal{V}\left(\Phi(L), R \hat{\otimes}_{\mathbf{R}} F_{y}, K\right) .
$$

Therefore the mapping

$$
\Phi^{*} \otimes \mathrm{id}:\left(\operatorname{Der}\left(R \hat{\otimes}_{\mathbf{R}} F_{y}\right)\right) * \otimes K \rightarrow L^{*} \otimes K
$$


induces a bijective mapping

$$
\mathscr{V}\left(\operatorname{Der}\left(R \hat{\otimes}_{\mathbf{R}} F_{y}\right), R \hat{\otimes}_{\mathbf{R}} F_{y}, K\right) \rightarrow \mathcal{V}(L, I, K) .
$$

We conclude that the closed ideal $I$ of $L$ is elliptic if and only if the closed ideal $R \hat{\otimes}_{\mathbf{R}} F_{y}$ of $\operatorname{Der}\left(R \hat{\otimes}_{\mathbf{R}} F_{y}\right)$ is elliptic. This last condition holds if and only if $R \hat{\otimes}_{\mathbf{R}} F_{y}$ is a finite-dimensional Lie algebra. We have thus proved:

Proposition 26.1. A non-abelian minimal closed ideal of a transitive Lie algebra of real type is elliptic if and only if it is finite-dimensional.

\section{References}

[1] N. Bourbaki, Éléments de mathématique, Topologie générale, Chapitre 2, Structures uniformes, $3^{\mathrm{e}}$ édition, Hermann, Paris, 1961.

[2] M. Buck, On the analyticity of equations defined by a class of transitive pseudogroups, Ph.D. thesis, Princeton University, 1974.

[3] C. Buttin \& P. Molino, Théorème général d'équivalence pour les pseudogroupes de Lie plats transitifs, J. Differential Geometry 9 (1974) 347-354.

[4] H. Cartan \& S. Eilenberg, Homological algebra, Princeton University Press, Princeton, 1956.

[5] J. F. Conn, A new class of counterexamples to the integrability problem, Proc. Nat. Acad. Sci. U.S.A. 74 (1977) 2655-2658.

[6] _ Non-abelian minimal closed ideals of transitive Lie algebras, Math. Notes, No. 25, Princeton University Press, Princeton, 1981.

[7] C. Freifeld, The cohomology of transitive filtered modules. I: The first cohomology group, Trans. Amer. Math. Soc. 144 (1969) 475-491.

[8] H. Goldschmidt, Existence theorems for analytic linear partial differential equations, Ann. of Math. 86 (1967) 246-270.

[9] _ Integrability criteria for systems of non-linear partial differential equations, J. Differential Geometry 1 (1967) 269-307.

[10] _ Prolongations of linear partial differential equations: I. A conjecture of Élie Cartan, Ann. Sci. École Norm. Sup. (4) 1 (1968) 417-444.

[11] _ Prolongations of linear partial differential equations. II. Inhomogeneous equations, Ann. Sci. École Norm. Sup. (4) 1 (1968) 617-625.

[12] __ Sur la structure des équations de Lie: I. Le troisième théorème fondamental, J. Differential Geometry 6 (1972) 357-373.

[13] __ Sur la structure des équations de Lie: II. Équations formellement transitives, J. Differential Geometry 7 (1972) 67-95.

[14] On the Spencer cohomology of a Lie equation, Partial Differential Equations (Proc. Sympos. Pure Math. Vol. XXIII, Berkeley, Calif., 1971), Amer. Math. Soc., 1973, 379-385.

[15] __ Prolongements d'équations différentielles linéaires. III. La suite exacte de cohomologie de Spencer, Ann. Sci. École Norm. Sup. (4) 7 (1974) 5-27.

[16] Sur la structure des équations de Lie: III. La cohomologie de Spencer, J. Differential Geometry 11 (1976) 167-223.

[17] The integrability problem for Lie equations, Bull. Amer. Math. Soc. 84 (1978) 531-546.

[18] H. Goldschmidt \& D. Spencer, Submanifolds and over-determined operators, Complex Analysis and Algebraic Geometry (A collection of papers dedicated to K. Kodaira), Iwanami Shoten Publishers, Tokyo, and Cambridge University Press, Cambridge, 1977, 319-356. 
[19] H. Goldschmidt \& S. Sternberg, The Hamilton-Cartan formalism in the calculus of variations, Ann. Inst. Fourier (Grenoble) 23 (1973) 203-267.

[20] V. W. Guillemin, A Jordan-Hölder decomposition for a certain class of infinite dimensional Lie algebras, J. Differential Geometry 2 (1968) 313-345.

[21] _ Infinite dimensional primitive Lie algebras, J. Differential Geometry 4 (1970) 257-282.

[22] V. W. Guillemin, D. Quillen \& S. Sternberg, The classification of the complex primitive pseudogroups, Proc. Nat. Acad. Sci. U.S.A. 55 (1966) 687-690.

[23] , The integrability of characteristics, Comm. Pure Appl. Math. 23 (1970) 39-77.

[24] V. W. Guillemin \& S. Sternberg, An algebraic model of transitive differential geometry, Bull. Amer. Math. Soc. 70 (1964) 16-47.

[25] _ Deformation theory of pseudogroup structures, Mem. Amer. Math. Soc., No. 64, 1966, $1-80$.

[26] The Lewy counterexample and the local equivalence problem for G-structures, J. Differential Geometry 1 (1967) 127-131.

[27] Notes on transitive Lie algebras, Polycopied notes, Harvard University.

[28] S. Helgason, Differential geometry and symmetric spaces, Academic Press, New York, 1962.

[29] K. Kodaira \& D. C. Spencer, Multifoliate structures, Ann. of Math. 74 (1961) 52-100.

[30] G. Köthe, Topologische lineare Räume. I, 2nd edition, Springer, Berlin, 1966.

[31] A. Kumpera \& D. Spencer, Lie equations. Volume I: general theory, Annals of Math. Studies, No. 73, Princeton University Press and University of Tokyo Press, Princeton, 1972.

[32] M. Kuranishi \& A. A. M. Rodrigues, Quotients of pseudo groups by invariant fiberings, Nagoya Math. J. 24 (1964) 109-128.

[33] B. Malgrange, Équations de Lie. I, II, J. Differential Geometry 6 (1972) 503-522; 7 (1972) $117-141$.

[34] T. Morimoto \& N. Tanaka, The classification of the real primitive infinite Lie algebras, J. Math. Kyoto Univ. 10 (1970) 207-243.

[35] A. S. Pollack, The integrability problem for pseudogroup structures, J. Differential Geometry 9 (1974) $355-390$.

[36] S. Shnider, The classification of real primitive infinite Lie algebras, J. Differential Geometry 4 (1970) 81-89.

[37] I. M. Singer \& S. Sternberg, The infinite groups of Lie and Cartan, Part I, (The transitive groups), J. Analyse Math. 15 (1965) 1-114.

[38] D. C. Spencer, Deformation of structures on manifolds defined by transitive, continuous pseudogroups, I, II, Ann. of Math. 76 (1962) 306-445.

[39] __ Overdetermined systems of linear partial differential equations, Bull. Amer. Math. Soc. 75 (1969) 179-239.

COLUmbia University 